\title{
Quantitative Estimates on the Binding Energy for Hydrogen in Non-Relativistic QED
}

\author{
Jean-Marie Barbaroux, Thomas Chen, \\ Vitali Vougalter and Semjon Vugalter
}

\begin{abstract}
In this paper, we determine the exact expression for the hydrogen binding energy in the Pauli-Fierz model up to the order $\alpha^{5} \log \alpha^{-1}$, where $\alpha$ denotes the fine structure constant, and prove rigorous bounds on the remainder term of the order $o\left(\alpha^{5} \log \alpha^{-1}\right)$. As a consequence, we prove that the binding energy is not a real analytic function of $\alpha$, and verify the existence of logarithmic corrections to the expansion of the ground state energy in powers of $\alpha$, as conjectured in the recent literature.
\end{abstract}

\section{Introduction}

For a hydrogen-like atom consisting of an electron interacting with a static nucleus of charge $e Z$, described by the Schrödinger Hamiltonian $-\Delta-\frac{\alpha Z}{|x|}$,

$$
\inf \sigma(-\Delta)-\inf \sigma\left(-\Delta-\frac{\alpha Z}{|x|}\right)=\frac{(Z \alpha)^{2}}{4} .
$$

corresponds to the binding energy necessary to remove the electron to spatial infinity.

The interaction of the electron with the quantized electromagnetic field is accounted for by adding to $-\Delta-\frac{\alpha Z}{|x|}$ the photon field energy operator $H_{f}$, and an operator $I(\alpha)$ which describes the coupling of the electron to the quantized electromagnetic field; the small parameter $\alpha$ is the fine-structure constant. Thereby, one obtains the Pauli-Fierz Hamiltonian described in detail in Sect. 2. The systematic study of this operator, in a more general case involving more than one electron, was initiated by Bach et al. [3-5].

In this case, determining the binding energy

$$
\inf \sigma\left(-\Delta+H_{f}+I(\alpha)\right)-\inf \sigma\left(-\Delta-\frac{\alpha Z}{|x|}+H_{f}+I(\alpha)\right)
$$


is a very hard problem. A main obstacle emerges from the fact that the ground-state energy is not an isolated eigenvalue of the Hamiltonian, and cannot be determined with ordinary perturbation theory. Furthermore, the photon form factor in the quantized electromagnetic vector potential occurring in the interaction term $I(\alpha)$ contains a critical frequency space singularity that is responsible for the infamous infrared problem in quantum electrodynamics. As a consequence, quantities such as the ground-state energy do not a priori exist as a convergent power series in the fine-structure constant $\alpha$ with coefficients independent of $\alpha$.

In recent years, several rigorous results addressing the computation of the binding energy have been obtained $[10,15,16]$. In particular, the coupling to the photon field has been shown to increase the binding energy of the electron to the nucleus, and that up to normal ordering, the leading term is $\frac{(\alpha Z)^{2}}{4}$ $[14,16,10]$.

Moreover, for a model with scalar bosons, the binding energy is determined in [15], in the first subleading order in powers of $\alpha$, up to $\alpha^{3}$, with error term $\alpha^{3} \log \alpha^{-1}$. This result has inspired the question of a possible emergence of logarithmic terms in the expansion of the binding energy; however, this question has so far remained open.

In [2], a sophisticated rigorous renormalization group analysis is developed in order to determine the ground-state energy (and the renormalized electron mass) up to any arbitrary precision in powers of $\alpha$, with an expansion of the form

$$
\varepsilon_{0}+\sum_{k=1}^{2 N} \varepsilon_{k}(\alpha) \alpha^{k / 2}+o\left(\alpha^{N}\right)
$$

(for any given $N$ ) where the coefficients $\varepsilon_{k}(\alpha)$ diverge as $\alpha \rightarrow 0$, but are smaller in magnitude than any positive power of $\alpha^{-1}$. The recursive algorithms developed in [2] are highly complex, and explicitly computing the ground-state energy to any subleading order in powers of $\alpha$ is an extensive task. While it is expected that the rate of divergence of these coefficient functions is proportional to a power of $\log \alpha^{-1}$, this is not explicitly exhibited in the current literature; for instance, it can a priori not be ruled out that terms involving logarithmic corrections cancel mutually.

The choice of atomic units in [2], inherited from [3], and motivated by the necessity to keep the $\alpha$-dependence only in the interaction term, but not in the Coulomb term, introduces a dependence between the ultraviolet cutoff and the fine-structure constant. This makes it challenging to compare the result derived for (2) to our estimate of the binding energy stated in Theorem 2.1, since we employ different units with an ultraviolet cutoff independent of $\alpha$. However, such comparison would require to apply the procedure used in this paper to the model described in [2].

The goal of the current paper is to develop an alternative method (as a continuation of [7]) that determines the binding energy up to several 
subleading orders in powers of $\alpha$, with rigorous error bounds, and proving the presence of terms logarithmic in $\alpha$.

The main result established in the present paper (for $Z=1$ ) states that the binding energy can be estimated as

$$
\frac{\alpha^{2}}{4}+e^{(1)} \alpha^{3}+e^{(2)} \alpha^{4}+e^{(3)} \alpha^{5} \log \alpha^{-1}+o\left(\alpha^{5} \log \alpha^{-1}\right),
$$

where $e^{(i)}(i=1,2,3)$ are independent of $\alpha, e^{(1)}>0$, and $e^{(3)} \neq 0$. Their explicit values are given in Theorem 2.1.

As a consequence, we conclude that the binding energy is not analytic in $\alpha$. In addition, our proof clarifies how the logarithmic factor in $\alpha^{5} \log \alpha^{-1}$ is linked to the infrared singularity of the photon form factor in the interaction term $I(\alpha)$. We note that for some models with a mild infrared behavior, [12], the ground-state energy is proven to be analytic in $\alpha$.

Notice that on the basis of the present computations, it is impossible to determine whether the $\alpha^{5} \log \alpha^{-1}$ term comes from inf $\sigma\left(-\Delta+H_{f}+I(\alpha)\right)$, or $\inf \sigma\left(-\Delta-\frac{\alpha Z}{|x|}+H_{f}+I(\alpha)\right)$ or both. However, this question has been recently answered in [6].

\section{Organization of the Proof}

The strategy consists mainly in an iteration procedure based on variational estimates. The derivation of the main result (3) is accomplished in two main steps, with first an estimate up to the order $\alpha^{3}$ with an error of the order $\mathcal{O}\left(\alpha^{4}\right)$, and then up to the order $\alpha^{5} \log \alpha$. Each of these steps requires both an upper and a lower bound on the binding energy. From the knowledge of an approximate ground state for $-\Delta+H_{f}+I(\alpha)$ (see [7]), the remaining hard task consists mainly in establishing a lower bound for the ground-state energy for $H$ yielding an upper bound for the binding energy. A lower bound for the binding energy can be obtained by choosing a bona fide trial state.

It is easy to see that the first term in the expansion for the binding energy is not smaller than the Coulomb energy $\frac{\alpha^{2}}{4}$ by taking a trial state, which is a product state of the electronic ground state $u_{\alpha}$ (15) of the Schrödinger-Coulomb operator $-\Delta-\frac{\alpha}{|x|}$ and the photon ground state of the self-energy operator given by (7) with zero total momentum. Indeed, as shown in [10] there is an increase of the binding energy. However, the first term is exactly the Coulomb energy [15].

The proof of the upper bound, up to the order $\alpha^{3}$ and to the order $\alpha^{5} \log \alpha^{-1}$ reduces to a minimization process.

Because of the soft photon problem, the set of minimizing sequences does not have a clear structure which helps to find the minimizer, or approximate minimizers. Therefore, prior to the above two steps, we have to appropriately restrict the set of states which we are looking at. For that purpose, we first establish, in Lemma 3.1, an a priori bound on the expected photon number in the ground state $\Psi^{\mathrm{GS}}$ of the Pauli-Fierz operator $\left(-\Delta-\frac{\alpha}{|x|}+H_{f}+I(\alpha)\right)$, namely $\left\langle\Psi^{\mathrm{GS}}, N_{f} \Psi^{\mathrm{GS}}\right\rangle=\mathcal{O}\left(\alpha^{2} \log \alpha^{-1}\right)$, where $N_{f}$ is the photon number operator. 
Moreover, in Theorem 4.1, we estimate the contribution to the binding energy stemming from states orthogonal to the ground state $u_{\alpha}$ of the Schrödinger operator. It enables us to show that the projection of the ground state $\Psi^{\mathrm{GS}}$ onto the subspace of functions orthogonal to $u_{\alpha}$ is small in several norms.

Estimate up to the order $\alpha^{3}$. To find a lower bound for the binding energy (Theorem 5.1), we pick a trial function $\Phi^{\text {trial }}$ which is the sum of two states. The first state is the product of the ground state $u_{\alpha}$ of the Schrödinger-Coulomb operator with the approximate ground state $\Psi_{0}$ of the self-energy operator (given by (7) below) with zero total momentum which was derived in [7]. The second state $\Phi_{\sharp}^{u_{\alpha}}(42)$ is a term orthogonal to $u_{\alpha}$ and has been chosen to minimize, up to the order $\alpha^{3}$, the sum of the cross term $\left\langle\left(-\Delta-\frac{\alpha}{|x|}+H_{f}+I(\alpha)\right) u_{\alpha} \Psi_{0}, \Phi_{\sharp}^{u_{\alpha}}\right\rangle$ and the term $\left\langle\left(H_{f}+P_{f}^{2}-\Delta-\frac{\alpha}{|x|}+\frac{\alpha^{2}}{4}\right) \Phi_{\sharp}^{u_{\alpha}}, \Phi_{\sharp}^{u_{\alpha}}\right\rangle$ stemming from the quadratic form for $H$ on the trial state $\Phi^{\text {trial }}=u_{\alpha} \Psi_{0}+\Phi_{\sharp}^{u_{\alpha}}$.

At that stage, we emphasize that the contribution of these two states yields not only an $\alpha^{3}$ term, but also an $\alpha^{5} \log \alpha^{-1}$ term, which is in fact the only contribution to the binding energy for this order as the detailed proof of the next order estimate shall show.

To recover the upper bound, we take an arbitrary state $\Psi$ satisfying the condition on the expected photon number. Then we consider $u_{\alpha} \Psi^{u_{\alpha}}$, the projection of this state onto $u_{\alpha}$, and $\Psi^{\perp}$ the projection onto the subspace orthogonal to $u_{\alpha}$. We estimate in Lemma 7.1 the quadratic form of $H$ on $u_{\alpha} \Psi^{u_{\alpha}}$, in Lemma 7.4 the quadratic form for $H$ on $\Psi^{\perp}$, and in Lemmata 7.2-7.3 the cross term. Collecting these estimates in (82) below yields that the function $\Psi$ can be an approximate minimizer up to the order $\alpha^{3}$, with error term $\mathcal{O}\left(\alpha^{4} \log \alpha^{-1}\right)$ of the functional $\langle H \Psi, \Psi\rangle$ only if the difference $R$ between this function $\Psi$ and the previous trial state $\Phi^{\text {trial }}$ satisfies the condition $\left\|H_{f}^{\frac{1}{2}} R\right\|^{2}=\mathcal{O}\left(\alpha^{4} \log \alpha^{-1}\right)$ (see $(87))$

According to Lemma 7.5, the last estimate itself allows to improve the expected photon number estimate for the ground state of $H$ given by Lemma 3.1, replacing there $\alpha^{2} \log \alpha^{-1}$ by $\alpha^{2}$. Then, repeating the above steps for the upper bound with this improved expected photon number yields the upper bound of the binding energy with an error $\mathcal{O}\left(\alpha^{4}\right)$, instead of $\mathcal{O}\left(\alpha^{4} \log \alpha^{-1}\right)$.

Estimate up to the order $\alpha^{5} \log \alpha^{-1}$. In order to derive the lower bound for the binding energy, we improve the previous trial function by adding several terms that where irrelevant for the estimate up to the order $\alpha^{3}$. Due to the improved estimates in Lemma 7.5, the expected photon number for the difference of the true ground state and this new trial function cannot exceed $\mathcal{O}\left(\alpha^{\frac{33}{16}}\right)$. Furthermore, we infer from Theorem 5.2 that this difference satisfies several smallness conditions. Using these conditions, we estimate once more the quadratic form and minimize it with respect to this difference in a similar way to what is done in Sect. 5 for the previous step.

The paper is organized as follows: In Sect. 2, we give a detailed definition of the model and state the main result. In Sect. 3 we establish an a priori 
bound on the expected photon number in the ground state of the Pauli-Fierz operator. In Sect. 4 we estimate the contribution to the binding energy stemming from states orthogonal to the ground state of the Schrödinger operator. The Sect. 5 is devoted to the statements of the lower and the upper bounds for the binding energy up to the order $\alpha^{3}$, as well as to the proof of the lower bound. The difficult part of the proof, namely the upper bound on the binding energy, is presented in four parts and postponed to Sect. 7. In Sects. 7.1-7.3, we estimate separately the terms according to the splitting of the variational state. We collect these results in Sect. 7.4 and establish then the proof of the upper bound up to the order $\alpha^{3}$. In Sect. 6, we establish the main steps of the proof of the upper bound for the binding energy up to the order $\alpha^{5} \log \alpha^{-1}$. We start with some useful definitions, the statement for the upper bound, and two propositions (proved in Appendices A and B), that gives estimates of the contributions to the binding energy according to a refined splitting of the variational state. Details of the remaining straightforward computations are given in Sect. 8. Finally, Sect. 9 provides the proof for a lower bound on the binding energy. In Appendix C, we provide some technical lemmata whose proof are straightforward but rather long.

\section{The Model}

We study a scalar electron interacting with the quantized electromagnetic field in the Coulomb gauge, and with the electrostatic potential generated by a fixed nucleus. The Hilbert space accounting for the Schrödinger electron is given by $\mathfrak{H}_{\mathrm{el}}=L^{2}\left(\mathbb{R}^{3}\right)$. The Fock space of photon states is given by

$$
\mathfrak{F}=\bigoplus_{n \in \mathbb{N}} \mathfrak{F}_{n}
$$

where the $n$-photon space $\mathfrak{F}_{n}=\bigotimes_{s}^{n}\left(L^{2}\left(\mathbb{R}^{3}\right) \otimes \mathbb{C}^{2}\right)$ is the symmetric tensor product of $n$ copies of one-photon Hilbert spaces $L^{2}\left(\mathbb{R}^{3}\right) \otimes \mathbb{C}^{2}$. The factor $\mathbb{C}^{2}$ accounts for the two independent transversal polarizations of the photon. On $\mathfrak{F}$, we introduce creation- and annihilation operators $a_{\lambda}^{*}(k), a_{\lambda}(k)$ satisfying the distributional commutation relations

$$
\left[a_{\lambda}(k), a_{\lambda^{\prime}}^{*}\left(k^{\prime}\right)\right]=\delta_{\lambda, \lambda^{\prime}} \delta\left(k-k^{\prime}\right), \quad\left[a_{\lambda}^{\sharp}(k), a_{\lambda^{\prime}}^{\sharp}\left(k^{\prime}\right)\right]=0,
$$

where $a_{\lambda}^{\sharp}$ denotes either $a_{\lambda}$ or $a_{\lambda}^{*}$. There exists a unique unit ray $\Omega_{f} \in \mathfrak{F}$, the Fock vacuum, which satisfies $a_{\lambda}(k) \Omega_{f}=0$ for all $k \in \mathbb{R}^{3}$ and $\lambda \in\{1,2\}$.

The Hilbert space of states of the system consisting of both the electron and the radiation field is given by

$$
\mathfrak{H}:=\mathfrak{H}_{\mathrm{el}} \otimes \mathfrak{F} .
$$

We use units such that $\hbar=c=1$, and where the mass of the electron equals $m=1 / 2$. The electron charge is then given by $e=\sqrt{\alpha}$, where the fine-structure constant $\alpha$ will here be considered as a small parameter.

Let $x \in \mathbb{R}^{3}$ be the position vector of the electron. 
We consider the normal ordered Pauli-Fierz Hamiltonian on $\mathfrak{H}$ for Hydrogen,

$$
:\left(i \nabla_{x} \otimes I_{f}-\sqrt{\alpha} A(x)\right)^{2}:+V(x) \otimes I_{f}+I_{\mathrm{el}} \otimes H_{f} .
$$

where : $\cdots$ : denotes normal ordering, corresponding to the subtraction of a normal ordering constant $c_{\text {n.o. }} \alpha$, with $c_{\text {n.o. }} I_{f}:=\left[A^{-}(x), A^{+}(x)\right]$ is independent of $x$.

The electrostatic potential $V(x)$ is the Coulomb potential for a static point nucleus of charge $e=\sqrt{\alpha}$ (i.e., $Z=1$ )

$$
V(x)=-\frac{\alpha}{|x|} .
$$

We will describe the quantized electromagnetic field by use of the Coulomb gauge condition.

The operator that couples an electron to the quantized vector potential is given by

$$
A(x)=\sum_{\lambda=1,2} \int_{\mathbb{R}^{3}} \frac{\chi_{\Lambda}(|k|)}{2 \pi|k|^{1 / 2}} \varepsilon_{\lambda}(k)\left[\mathrm{e}^{i k x} \otimes a_{\lambda}(k)+\mathrm{e}^{-i k x} \otimes a_{\lambda}^{*}(k)\right] \mathrm{d} k
$$

where by the Coulomb gauge condition, $\operatorname{div} A=0$.

The vectors $\varepsilon_{\lambda}(k) \in \mathbb{R}^{3}$ are the two orthonormal polarization vectors perpendicular to $k$,

$$
\varepsilon_{1}(k)=\frac{\left(k_{2},-k_{1}, 0\right)}{\sqrt{k_{1}^{2}+k_{2}^{2}}} \quad \text { and } \quad \varepsilon_{2}(k)=\frac{k}{|k|} \wedge \varepsilon_{1}(k) .
$$

The function $\chi_{\Lambda}$ implements an ultraviolet cutoff on the momentum $k$. We assume $\chi_{\Lambda}$ to be of class $C^{1}$, with compact support in $\{|k| \leq \Lambda\}, \chi_{\Lambda} \leq 1$ and $\chi_{\Lambda}=1$ for $|k| \leq \Lambda-1$. For convenience, we shall write

$$
A(x)=A^{-}(x)+A^{+}(x),
$$

where

$$
A^{-}(x)=\sum_{\lambda=1,2} \int_{\mathbb{R}^{3}} \frac{\chi_{\Lambda}(|k|)}{2 \pi|k|^{1 / 2}} \varepsilon_{\lambda}(k) \mathrm{e}^{i k x} \otimes a_{\lambda}(k) \mathrm{d} k
$$

is the part of $A(x)$ containing the annihilation operators, and $A^{+}(x)=$ $\left(A^{-}(x)\right)^{*}$.

The photon field energy operator $H_{f}$ is given by

$$
H_{f}=\sum_{\lambda=1,2} \int_{\mathbb{R}^{3}}|k| a_{\lambda}^{*}(k) a_{\lambda}(k) \mathrm{d} k .
$$

We will, with exception of our discussion in Sect. 3, study the unitarily equivalent Hamiltonian

$$
H=U\left(:\left(i \nabla_{x} \otimes I_{f}-\sqrt{\alpha} A(x)\right)^{2}:+V(x) \otimes I_{f}+I_{\mathrm{el}} \otimes H_{f}\right) U^{*},
$$


where the unitary transform $U$ is defined by

$$
U=\mathrm{e}^{i P_{f} \cdot x}
$$

and

$$
P_{f}=\sum_{\lambda=1,2} \int k a_{\lambda}^{*}(k) a_{\lambda}(k) \mathrm{d} k
$$

is the photon momentum operator. We have

$$
U i \nabla_{x} U^{*}=i \nabla_{x}+P_{f} \quad \text { and } \quad U A(x) U^{*}=A(0) .
$$

In addition, the Coulomb operator $V$, the photon field energy $H_{f}$, and the photon momentum $P_{f}$ remain unchanged under the action of $U$. Therefore, in this new system of variables, the Hamiltonian reads as follows:

$$
H=:\left(\left(i \nabla_{x} \otimes I_{f}-I_{\mathrm{el}} \otimes P_{f}\right)-\sqrt{\alpha} A(0)\right)^{2}:+H_{f}-\frac{\alpha}{|x|},
$$

where : ... : denotes again the normal ordering. Notice that the operator $H$ can be rewritten, taking into account the normal ordering and omitting, by abuse of notations, the operators $I_{\mathrm{el}}$ and $I_{f}$,

$$
\begin{aligned}
H= & \left(-\Delta_{x}-\frac{\alpha}{|x|}\right)+\left(H_{f}+P_{f}^{2}\right)-2 \operatorname{Re}\left(i \nabla_{x} \cdot P_{f}\right) \\
& -2 \sqrt{\alpha}\left(i \nabla_{x}-P_{f}\right) \cdot A(0)+2 \alpha A^{+}(0) \cdot A^{-}(0)+2 \alpha \operatorname{Re}\left(A^{-}(0)\right)^{2} .
\end{aligned}
$$

For a free spinless electron coupled to the quantized electromagnetic field, the self-energy operator $T$ is given by

$$
T=:\left(i \nabla_{x} \otimes I_{f}-\sqrt{\alpha} A(x)\right)^{2}:+I_{\mathrm{el}} \otimes H_{f} .
$$

We note that this system is translationally invariant; that is, $T$ commutes with the operator of total momentum

$$
P_{\mathrm{tot}}=p_{\mathrm{el}} \otimes I_{f}+I_{\mathrm{el}} \otimes P_{f},
$$

where $p_{\mathrm{el}}$ and $P_{f}$ denote, respectively, the electron and the photon momentum operators.

Let $\mathbb{C} \otimes \mathfrak{F}$ be the fiber Hilbert space corresponding to conserved total momentum $p \in \mathbb{R}^{3}$.

For fixed value $p$ of the total momentum, the restriction of $T$ to the fibre space is given by (see, e.g. [8])

$$
T(p)=:\left(p-P_{f}-\sqrt{\alpha} A(0)\right)^{2}:+H_{f}
$$

where by abuse of notation, we again dropped all tensor products involving the identity operators $I_{f}$ and $I_{\mathrm{el}}$. Henceforth, we will write

$$
A^{ \pm} \equiv A^{ \pm}(0) \text {. }
$$

Moreover, we denote

$$
\Sigma_{0}=\inf \sigma(T) \quad \text { and } \quad \Sigma=\inf \sigma(H)=\inf \sigma(T+V) .
$$

It is proven in $[1,8]$ that $\Sigma_{0}=\inf \sigma(T(0))$ is an eigenvalue of the operator $T(0)$. 
Our main result is the following theorem:

Theorem 2.1. The binding energy fulfills

$$
\Sigma_{0}-\Sigma=\frac{1}{4} \alpha^{2}+e^{(1)} \alpha^{3}+e^{(2)} \alpha^{4}+e^{(3)} \alpha^{5} \log \alpha^{-1}+o\left(\alpha^{5} \log \alpha^{-1}\right),
$$

where

$$
e^{(1)}=\frac{2}{\pi} \int_{0}^{\infty} \frac{\chi_{\Lambda}^{2}(t)}{1+t} \mathrm{~d} t,
$$

$$
\begin{aligned}
& e^{(2)}= \frac{2}{3} \operatorname{Re} \sum_{i=1}^{3}\left\langle\left(A^{-}\right)^{i}\left(H_{f}+P_{f}^{2}\right)^{-1} A^{+} . A^{+} \Omega_{f},\left(H_{f}+P_{f}^{2}\right)^{-1}\left(A^{+}\right)^{i} \Omega_{f}\right\rangle \\
&+\frac{1}{3} \sum_{i=1}^{3} \|\left(H_{f}+P_{f}^{2}\right)^{-\frac{1}{2}}\left(2 A^{+} . P_{f}\left(H_{f}+P_{f}^{2}\right)^{-1}\left(A^{+}\right)^{i}\right. \\
&\left.-P_{f}^{i}\left(H_{f}+P_{f}^{2}\right)^{-1} A^{+} \cdot A^{+}\right) \Omega_{f} \|^{2} \\
&-\frac{2}{3} \sum_{i=1}^{3}\left\|A^{-}\left(H_{f}+P_{f}^{2}\right)^{-1}\left(A^{+}\right)^{i} \Omega_{f}\right\|^{2}+4 a_{0}^{2}\left\|Q_{1}^{\perp}\left(-\Delta-\frac{1}{|x|}+\frac{1}{4}\right)^{-\frac{1}{2}} \Delta u_{1}\right\|^{2}, \\
& a_{0}=\int \frac{k_{1}^{2}+k_{2}^{2}}{4 \pi^{2}|k|^{3}} \frac{2}{|k|^{2}+|k|} \chi_{\Lambda}(|k|) \mathrm{d} k_{1} \mathrm{~d} k_{2} \mathrm{~d} k_{3}, \\
& e^{(3)}=-\frac{1}{3 \pi}\left\|\left(-\Delta-\frac{1}{|x|}+\frac{1}{4}\right)^{\frac{1}{2}} \nabla u_{1}\right\| \|^{2},
\end{aligned}
$$

and $Q_{1}^{\perp}$ is the projection onto the orthogonal complement to the ground state $u_{1}$ of the Schrödinger operator $-\Delta-\frac{1}{|x|}($ for $\alpha=1)$.

\section{Bounds on the Expected Photon Number}

Lemma 3.1. Let

$$
K=(i \nabla-\sqrt{\alpha} A(x))^{2}+H_{f}-\frac{\alpha}{|x|}=v^{2}+H_{f}-\frac{\alpha}{|x|},
$$

be the Pauli-Fierz operator defined without normal ordering, where $v=i \nabla-$ $\sqrt{\alpha} A(x)$. Let $\Psi \in \mathcal{H}$ denote the ground state of $K$,

$$
K \Psi=E \Psi
$$

normalized by

$$
\|\Psi\|=1 \text {. }
$$

Let

$$
N_{f}:=\sum_{\lambda=1,2} \int a_{\lambda}^{*}(k) a_{\lambda}(k) \mathrm{d} k
$$

denote the photon number operator. Then, there exists a constant $c$ independent of $\alpha$, such that for any sufficiently small $\alpha>0$, the estimate 


$$
\left\langle\Psi, N_{f} \Psi\right\rangle \leq c \alpha^{2} \log \alpha^{-1}
$$

is satisfied.

Proof. Using

$$
\left[a_{\lambda}(k), H_{f}\right]=|k| a_{\lambda}(k), \quad \text { and } \quad\left[a_{\lambda}(k), v\right]=\sqrt{(\alpha)} \frac{\epsilon(k)}{2 \pi|k|^{\frac{1}{2}}} \chi_{\lambda}(k) \mathrm{e}^{i k \cdot x}
$$

and the pull-through formula,

$$
\begin{aligned}
a_{\lambda}(k) E \Psi & =a_{\lambda}(k) K \Psi \\
& =\left[\left(H_{f}+|k|\right) a_{\lambda}(k)-\frac{1}{|x|} a_{\lambda}(k)+\left[v, a_{\lambda}(k)\right] v+v\left[v, a_{\lambda}(k)\right]\right] \Psi,
\end{aligned}
$$

we get

$$
a_{\lambda}(k) \Psi=-\frac{\sqrt{\alpha} \chi_{\Lambda}(|k|)}{2 \pi \sqrt{|k|}} \frac{2}{K+|k|-E}\left((i \nabla-\sqrt{\alpha} A(x)) \cdot \epsilon_{\lambda}(k) \mathrm{e}^{i k \cdot x}\right) \Psi .
$$

From (9), we obtain

$$
\begin{aligned}
\left\|a_{\lambda}(k) \Psi\right\|^{2} & \leq \frac{\alpha \chi_{\Lambda}(|k|)}{\pi^{2}|k|}\left\|\frac{1}{K+|k|-E}\right\|^{2}\|(i \nabla-\sqrt{\alpha} A(x)) \Psi\|^{2} \\
& \leq \frac{\alpha \chi_{\Lambda}(|k|)}{\pi^{2}|k|^{3}}\left[\langle\Psi, K \Psi\rangle+\left\langle\Psi, \frac{\alpha}{|x|} \Psi\right\rangle\right]
\end{aligned}
$$

since $K-E \geq 0$, and $(i \nabla-\sqrt{\alpha} A(x))^{2} \leq\left(K+\frac{\alpha}{|x|}\right)$. we have

Since $\langle\Psi,: K: \Psi\rangle=-\alpha^{2} / 4+o\left(\alpha^{2}\right)([10,14,16])$ and $: K:=K-c_{\text {n.o. }} \alpha$,

$$
\langle\Psi, K \Psi\rangle \leq c_{\text {n.o. }} \alpha .
$$

Moreover, for the normal ordered hamiltonian defined in (4), we have

$$
\begin{aligned}
: K: & =-\Delta_{x}-4 \sqrt{\alpha} \operatorname{Re}\left(i \nabla_{x} \cdot A^{-}(x)\right)+\alpha: A(x)^{2}:+H_{f}-\frac{\alpha}{|x|} \\
& \geq(1-c \sqrt{\alpha})\left(-\Delta_{x}\right)+\alpha: A(x)^{2}:+(1-c \sqrt{\alpha}) H_{f}-\frac{\alpha}{|x|} \\
& \geq-c \alpha^{2}-\frac{1}{2} \Delta_{x}-\frac{\alpha}{|x|}=-c \alpha^{2}+2\left(-\frac{1}{4} \Delta_{x}-\frac{\alpha}{|x|}\right)+\frac{\alpha}{|x|} .
\end{aligned}
$$

where in the last inequality we used (see, e.g. [13])

$$
\alpha: A(x)^{2}:+(1-c \sqrt{\alpha}) H_{f} \geq-c \alpha^{2} .
$$

Since

$$
-\frac{1}{4} \Delta_{x}-\frac{\alpha}{|x|} \geq-4 \alpha^{2}
$$

inequality (11) implies

$$
\left\langle\Psi, \frac{\alpha}{|x|} \Psi\right\rangle \leq\left\langle-\Delta_{x} \Psi, \Psi\right\rangle+\frac{1}{4} \alpha^{2}\|\Psi\|^{2} \leq c \alpha^{2}\|\Psi\|^{2} .
$$


Collecting (10), (12) and (9) we find

$$
\left\|a_{\lambda}(k) \Psi\right\| \leq \frac{c \alpha \chi_{\Lambda}(|k|)}{|k|^{\frac{3}{2}}} .
$$

This a priori bound exhibits the $L^{2}$-critical singularity in frequency space. It does not take into consideration the exponential localization of the ground state due to the confining Coulomb potential and appears in a similar form for the free electron.

To account for the latter, we use the following two results from the work of Griesemer et al. [13]. Equation (58) in [13] provides the bound:

$$
\left\|a_{\lambda}(k) \Psi\right\|<\frac{c \sqrt{\alpha} \chi_{\Lambda}(|k|)}{|k|^{\frac{1}{2}}}\||x| \Psi\| .
$$

Moreover, Lemma 6.2 in [13] states that

$$
\|\exp [\beta|x|] \Psi\|^{2} \leq c\left[1+\frac{1}{\Sigma_{0}-E-\beta^{2}}\right]\|\Psi\|^{2},
$$

for any

$$
\beta^{2}<\Sigma_{0}-E=O\left(\alpha^{2}\right) .
$$

For the 1-electron case, $\Sigma_{0}$ is the infimum of the self-energy operator, and $E$ is the ground-state energy of : $K$ :. Choosing $\beta=O(\alpha)$,

$$
\begin{aligned}
\||x| \Psi\| & \leq\left\||x|^{4} \Psi\right\|^{\frac{1}{4}}\|\Psi\|^{\frac{3}{4}} \leq \frac{(4 !)^{\frac{1}{4}}}{\beta}\|\exp [\beta|x|] \Psi\|^{\frac{1}{4}}\|\Psi\|^{\frac{3}{4}} \\
& \leq \frac{c}{\beta}\left[1+\frac{1}{\Sigma_{0}-E-\beta^{2}}\right]^{\frac{1}{8}}\|\Psi\| \\
& \leq c_{1} \alpha^{-\frac{5}{4}} .
\end{aligned}
$$

Notably, this bound only depends on the binding energy of the potential.

Thus,

$$
\left\|a_{\lambda}(k) \Psi\right\|<\frac{c \alpha^{-\frac{3}{4}} \chi_{\Lambda}(|k|)}{|k|^{\frac{1}{2}}} .
$$

We see that binding to the Coulomb potential weakens the infrared singularity by a factor $|k|$, but at the expense of a large constant factor $\alpha^{-2}$. For the free electron, this estimate does not exist.

Using (13) and (14), we find

$$
\begin{aligned}
\left\langle\Psi, N_{f} \Psi\right\rangle & =\int \mathrm{d} k\left\|a_{\lambda}(k) \Psi\right\|^{2} \\
& \leq \int_{|k|<\delta} \mathrm{d} k \frac{c \alpha^{-\frac{3}{2}}}{|k|}+\int_{\delta \leq|k| \leq \Lambda} \mathrm{d} k \frac{c \alpha^{2}}{|k|^{3}}
\end{aligned}
$$




$$
\begin{aligned}
& \leq c \alpha^{-\frac{3}{2}} \delta^{2}+c^{\prime} \alpha^{2} \log \frac{1}{\delta} \\
& \leq c \alpha^{\frac{9}{4}}+c^{\prime \prime} \alpha^{2} \log \alpha^{-1} .
\end{aligned}
$$

for $\delta=\alpha^{\frac{15}{8}}$. This proves the lemma.

\section{Estimates on the Quadratic Form for States Orthogonal to the Ground State of the Schrödinger Operator}

Throughout this paper, we will denote by $\pi^{n}$ the projection onto the $n$th photon sector (without distinction for the $n$-photon sector of $\mathfrak{F}$ and the $n$-photon sector of $\mathfrak{H})$. We also define $\pi^{\geq n}=1-\sum_{j=0}^{n-1} \pi^{j}$.

Starting with this section, we study the Hamiltonian $H$ defined in (6), written in relative coordinates. In particular, $i \nabla_{x}$ now stands for the operator unitarily equivalent to the operator of total momentum, which, by abuse of notation, will be denoted by $P$.

Let

$$
u_{\alpha}(x)=\frac{1}{\sqrt{8 \pi}} \alpha^{3 / 2} \mathrm{e}^{-\alpha|x| / 2}
$$

be the normalized ground state of the Schrödinger operator

$$
h_{\alpha}:=-\Delta_{x}-\frac{\alpha}{|x|} \text {. }
$$

We will also denote by $-e_{0}=-\frac{\alpha^{2}}{4}$ and $-e_{1}=-\frac{\alpha^{2}}{16}$ the two lowest eigenvalues of this operator.

Theorem 4.1. Assume that $\Phi \in \mathfrak{H}$ fulfils $\left\langle\pi^{k} \Phi, u_{\alpha}\right\rangle_{L^{2}\left(\mathbb{R}^{3}, \mathrm{~d} x\right)}=0$, for all $k \geq 0$. Then there exists $1>\nu>0$ and $\alpha_{0}>0$ such that for all $0<\alpha<\alpha_{0}$

$$
\langle H \Phi, \Phi\rangle \geq\left(\Sigma_{0}-e_{0}\right)\|\Phi\|^{2}+\delta\|\Phi\|^{2}+\nu\left\|H_{f}^{\frac{1}{2}} \Phi\right\|^{2}
$$

where $\delta=\left(e_{0}-e_{1}\right) / 2=\frac{3}{32} \alpha^{2}$.

Remark 4.1. All photons with momenta larger than the ultraviolet cutoff do not contribute to lower the energy. More precisely, due to the cutoff function $\chi_{\Lambda}(|k|)$ in the definition of $A(x)$, and since we have

$$
H=\left(i \nabla_{x}-P_{f}\right)^{2}-2 \sqrt{\alpha} \operatorname{Re}\left(i \nabla_{x}-P_{f}\right) \cdot A(0)+\alpha: A(0)^{2}:+H_{f}-\frac{\alpha}{|x|},
$$

it follows that for any given normalized state $\Phi \in \mathfrak{H}$, there exists a normalized state $\Phi_{\leq \Lambda}$ such that $\forall x \in \mathbb{R}^{3}$, for all $n \in\{1,2, \ldots\}$, for all $\left(\left(k_{1}, \lambda_{1}\right)\right.$, $\left.\left(k_{2}, \lambda_{2}\right), \ldots,\left(k_{n}, \lambda_{n}\right)\right) \in\left(\left(\mathbb{R}^{3} \backslash\{k,|k| \leq \Lambda+1\}\right) \times\{1,2\}\right)^{n}$, we have

$$
\pi^{n} \Phi_{\leq \Lambda}\left(x,\left(k_{1}, \lambda_{1}\right),\left(k_{2}, \lambda_{2}\right), \ldots,\left(k_{n}, \lambda_{n}\right)\right)=0
$$

and

$$
\left\langle\Phi_{\leq \Lambda}, H \Phi_{\leq \Lambda}\right\rangle \leq\langle\Phi, H \Phi\rangle \quad \text { and } \quad\left\langle\Phi_{\leq \Lambda}, T \Phi_{\leq \Lambda}\right\rangle \leq\langle\Phi, T \Phi\rangle .
$$


A key consequence of this remark is that throughout the paper, all states will be implicitly assumed to fulfill condition (17). This is crucial for the proof of Corollary 4.2.

To prove Theorem 4.1, we first need the following Lemma:

Lemma 4.1. There exists $c_{0}>0$ such that for all $\alpha$ small enough we have

$$
H-\frac{1}{2}\left(P-P_{f}\right)^{2}-\frac{1}{2} H_{f} \geq-c_{0} \alpha^{2} .
$$

A straightforward consequence of this lemma is the following result:

Corollary 4.1. Let $\Psi^{G S}$ be the normalized ground state of $H$. Then

$$
\left\langle H_{f} \Psi^{G S}, \Psi^{G S}\right\rangle \leq 2 c_{0} \alpha^{2}\left\|\Psi^{G S}\right\|^{2}
$$

Proof. This follows from $\left\langle H \Psi^{\mathrm{GS}}, \Psi^{\mathrm{GS}}\right\rangle \leq\left(\Sigma_{0}-e_{0}\right)\left\|\Psi^{\mathrm{GS}}\right\|^{2}<0$. The last inequality holds since $e_{0}=-\alpha^{2} / 4$ and $\Sigma_{0}$ is the infimum for the normal ordered Hamiltonian for the free electron, and thus $\Sigma_{0} \leq 0$.

Moreover, from Theorem 4.1 and Lemma 4.1, we obtain

Corollary 4.2. Assume that $\Phi \in \mathfrak{H}$ is such that $\left\langle\pi^{n} \Phi, u_{\alpha}\right\rangle_{L^{2}\left(\mathbb{R}^{3}, \mathrm{~d} x\right)}=0$ holds for all $n \geq 0$. Then, for $\nu$ and $\delta$ defined in Theorem 4.1, there exists $\zeta>0$, and $\alpha_{0}>0$ such that for all $0<\alpha<\alpha_{0}$ we have

$$
\langle H \Phi, \Phi\rangle \geq\left(\Sigma_{0}-e_{0}\right)\|\Phi\|^{2}+M[\Phi],
$$

where

$$
M[\Phi]:=\frac{\delta}{2}\|\Phi\|^{2}+\frac{\nu}{2}\left\|H_{f}^{\frac{1}{2}} \Phi\right\|^{2}+\zeta\left\|\left(P-P_{f}\right) \Phi\right\|^{2}+\zeta\left\|\pi^{n \leq 4} P \Phi\right\|^{2} .
$$

Proof. According to Remark 4.1, there exists $\widetilde{c}>1$ such that the operator inequality $P_{f}^{2} \pi^{n \leq 4} \leq \widetilde{c} H_{f} \pi^{n \leq 4}$ holds on the set of states for which (17) is satisfied. The value of $\widetilde{c}$ only depends on the ultraviolet cutoff $\Lambda$. Thus,

$$
\begin{aligned}
\left\|\pi^{n \leq 4} P \Phi\right\|^{2} & \leq 2\left\|\left(P-P_{f}\right) \Phi\right\|^{2}+2\left\|\pi^{n \leq 4} P_{f} \Phi\right\|^{2} \\
& \leq 2\left\|\left(P-P_{f}\right) g\right\|^{2}+2 \widetilde{c}\left\|H_{f}^{\frac{1}{2}} \pi^{n \leq 4} \Phi\right\|^{2},
\end{aligned}
$$

which yields

$$
\left\|\left(P-P_{f}\right) \Phi\right\|^{2} \geq \frac{1}{2}\left\|\pi^{n \leq 4} P \Phi\right\|^{2}-\widetilde{c}\left\|H_{f}^{\frac{1}{2}} \pi^{n \leq 4} \Phi\right\|^{2} .
$$

Therefore, it suffices to prove Corollary 4.2 with $M[\Phi]$ replaced by

$$
\widetilde{M}[\Phi]:=\frac{\delta}{2}\|\Phi\|^{2}+\frac{3}{4} \nu\left\|H_{f}^{\frac{1}{2}} \Phi\right\|^{2}+2 \zeta\left\|\left(P-P_{f}\right) \Phi\right\|^{2},
$$

and $\zeta$ small enough so that $\widetilde{c} \zeta<\frac{\nu}{4}$.

Now we consider two cases. Let $c_{1}:=\max \left\{8 c_{0}, 8 \delta / \alpha^{2}\right\}$.

If $\left\|\left(P-P_{f}\right) \Phi\right\|^{2} \leq c_{1} \alpha^{2}\|\Phi\|^{2}$, Theorem 4.1 and the above remark imply (19). 
If $\left\|\left(P-P_{f}\right) \Phi\right\|^{2}>c_{1} \alpha^{2}\|\Phi\|^{2}$, Lemma 4.1 implies that

$$
\begin{aligned}
\langle H \Phi, \Phi\rangle & \geq \frac{1}{2}\left\langle\left(P-P_{f}\right)^{2} \Phi, \Phi\right\rangle+\frac{1}{2}\left\langle H_{f} \Phi, \Phi\right\rangle-c_{0} \alpha^{2}\|\Phi\|^{2} \\
& \geq \frac{1}{4}\left\langle\left(P-P_{f}\right)^{2} \Phi, \Phi\right\rangle+\frac{1}{2}\left\langle H_{f} \Phi, \Phi\right\rangle+\frac{1}{8} c_{1} \alpha^{2}\|\Phi\|^{2},
\end{aligned}
$$

which concludes the proof since $\Sigma_{0}-e_{0}<0$.

Proof of Lemma 4.1. Recall the notation $A^{ \pm} \equiv A^{ \pm}(0)$. The following holds:

$$
\begin{aligned}
H- & \frac{1}{2}\left(P-P_{f}\right)^{2}-\frac{1}{2} H_{f} \\
= & \frac{1}{2}\left(P-P_{f}\right)^{2}-\frac{\alpha}{|x|}-2 \sqrt{\alpha} \operatorname{Re}\left(\left(P-P_{f}\right) \cdot A(0)\right)+2 \alpha \operatorname{Re}\left(A^{-}\right)^{2} \\
+ & +2 \alpha A^{+} \cdot A^{-}+\frac{1}{2} H_{f}, \\
& \frac{1}{4}\left(P-P_{f}\right)^{2}-\frac{\alpha}{|x|} \geq-4 \alpha^{2} .
\end{aligned}
$$

and

$$
2 \sqrt{\alpha}\left|\left\langle\left(P-P_{f}\right) \cdot A(0) \psi, \psi\right\rangle\right| \leq 2 \sqrt{\alpha}\left\|\left(P-P_{f}\right) \psi\right\|^{2}+2 \sqrt{\alpha}\left\|A^{-} \psi\right\|^{2} .
$$

By the Schwarz inequality, there exists $c_{1}$ independent of $\alpha$ such that

$$
\left\|A^{-} \psi\right\|^{2} \leq c_{1}\left\|H_{f}^{\frac{1}{2}} \psi\right\|^{2} .
$$

Inequalities (23)-(24) imply that for small $\alpha$,

$$
2 \sqrt{\alpha}\left|\left\langle\left(P-P_{f}\right) \cdot A(0) \psi, \psi\right\rangle\right| \leq \frac{1}{4}\left\|\left(P-P_{f}\right) \psi\right\|^{2}+\frac{1}{4}\left\langle H_{f} \psi, \psi\right\rangle .
$$

Moreover, using (24) and

$$
\left\|A^{+} \psi\right\|^{2} \leq c_{2}\|\psi\|^{2}+c_{3}\left\|H_{f}^{\frac{1}{2}} \psi\right\|^{2},
$$

we arrive at

$$
\begin{aligned}
\alpha\left\langle\left(A^{-}\right)^{2} \psi, \psi\right\rangle & =\alpha\left\langle A^{-} \psi, A^{+} \psi\right\rangle \leq \epsilon\left\|A^{-} \psi\right\|^{2}+\epsilon^{-1} \alpha^{2}\left\|A^{+} \psi\right\|^{2} \\
& \leq \epsilon c_{1}\left\|H_{f}^{\frac{1}{2}} \psi\right\|^{2}+\epsilon^{-1} \alpha^{2}\left(c_{2}\|\psi\|^{2}+c_{3}\left\|H_{f}^{\frac{1}{2}} \psi\right\|^{2}\right) .
\end{aligned}
$$

Collecting the inequalities (22), (25) and (27) with $\epsilon<1 /\left(8 c_{1}\right)$ and $\alpha$ small enough, completes the proof.

Proof of Theorem 4.1. Let $\Phi:=\Phi_{1}+\Phi_{2}:=\chi\left(|P|<\frac{p_{c}}{2}\right) \Phi+\chi\left(|P| \geq \frac{p_{c}}{2}\right) \Phi$, where $P=i \nabla_{x}$ is the total momentum operator (due to the transformation (5)) and $p_{c}=\frac{1}{3}$ is a lower bound on the norm of the total momentum for which $[8$, Theorem 3.2] holds. 
Since $P$ commutes with the translation invariant operator $H+\frac{\alpha}{|x|}$, we have for all $\epsilon \in(0,1)$,

$$
\begin{aligned}
\langle H \Phi, \Phi\rangle & =\left\langle H \Phi_{1}, \Phi_{1}\right\rangle+\left\langle H \Phi_{2}, \Phi_{2}\right\rangle-2 \operatorname{Re}\left\langle\frac{\alpha}{|x|} \Phi_{1}, \Phi_{2}\right\rangle \\
& \geq\left\langle H \Phi_{1}, \Phi_{1}\right\rangle+\left\langle H \Phi_{2}, \Phi_{2}\right\rangle-\epsilon\left\langle\frac{\alpha}{|x|} \Phi_{1}, \Phi_{1}\right\rangle-\epsilon^{-1}\left\langle\frac{\alpha}{|x|} \Phi_{2}, \Phi_{2}\right\rangle .
\end{aligned}
$$

- First, we have the following estimate:

$$
\begin{aligned}
: & \left(P-P_{f}-\sqrt{\alpha} A(0)\right)^{2}:+H_{f} \\
\quad & =\left(P-P_{f}\right)^{2}-2 \operatorname{Re}\left(P-P_{f}\right) \cdot \sqrt{\alpha} A(0)+\alpha: A(0)^{2}:+H_{f} \\
& \geq(1-\sqrt{\alpha})\left(P-P_{f}\right)^{2}+(\alpha-\sqrt{\alpha}): A(0)^{2}:+H_{f}-c_{\text {n.o. }} \sqrt{\alpha} \\
& \geq(1-\sqrt{\alpha})\left(P-P_{f}\right)^{2}+(1-\mathcal{O}(\sqrt{\alpha})) H_{f}-\mathcal{O}(\sqrt{\alpha})
\end{aligned}
$$

where in the last inequality we used (24) and (26). Therefore,

$$
\begin{aligned}
& \left\langle\left(H-\epsilon^{-1} \frac{\alpha}{|x|}\right) \Phi_{2}, \Phi_{2}\right\rangle \geq\left\langle\left(\frac{1-\sqrt{\alpha}}{2}\left(P-P_{f}\right)^{2}-\left(1+\epsilon^{-1}\right) \frac{\alpha}{|x|}\right) \Phi_{2}, \Phi_{2}\right\rangle \\
& \quad+\left\langle\left(\frac{1-\sqrt{\alpha}}{2}\left(P-P_{f}\right)^{2}+(1-\mathcal{O}(\sqrt{\alpha})) H_{f}-\mathcal{O}(\sqrt{\alpha})\right) \Phi_{2}, \Phi_{2}\right\rangle
\end{aligned}
$$

The lowest eigenvalue of the Schrödinger operator $-(1-\mathcal{O}(\sqrt{\alpha})) \frac{\Delta}{2}-\frac{\left(1+\epsilon^{-1}\right) \alpha}{|x|}$ is larger than $-c_{\epsilon} \alpha^{2}$. Thus, using (29) and denoting

$$
L:=\frac{1-\sqrt{\alpha}}{2}\left(P-P_{f}\right)^{2}+(1-\mathcal{O}(\sqrt{\alpha})) H_{f}-\mathcal{O}(\sqrt{\alpha})-c_{\epsilon} \alpha^{2},
$$

we get

$$
\left\langle H \Phi_{2}, \Phi_{2}\right)-\epsilon^{-1}\left\langle\frac{\alpha}{|x|} \Phi_{2}, \Phi_{2}\right\rangle \geq\left(L \Phi_{2}, \Phi_{2}\right\rangle .
$$

Now we have the following alternative: Either $\left|P_{f}\right|<\frac{p_{c}}{3}$, in which case we have $\left\langle L \Phi_{2}, \Phi_{2}\right\rangle \geq\left(\frac{p_{c}^{2}}{24}-\mathcal{O}(\sqrt{\alpha})\right)\left\|\Phi_{2}\right\|^{2}$, or $\left|P_{f}\right| \geq \frac{p_{c}}{3}$, in which case, using $\Phi_{2}=\chi\left(|P|>\frac{p_{c}}{2}\right) \Phi_{2}$ and $H_{f} \geq\left|P_{f}\right|$, we have $L \geq\left(\frac{p_{c}}{6}-\mathcal{O}(\sqrt{\alpha})\right)\left\|\Phi_{2}\right\|^{2}$. In both cases, for $\alpha$ small enough, this yields the bound

$$
\left\langle L \Phi_{2}, \Phi_{2}\right\rangle \geq \frac{p_{c}^{2}}{48}\left\|\Phi_{2}\right\|^{2} \geq\left(\Sigma_{0}-e_{0}+\frac{7}{8}\left(e_{0}-e_{1}\right)\right)\left\|\Phi_{2}\right\|^{2}
$$

since, for $\alpha$ small enough, the right-hand side tends to zero, whereas $p_{c}$ is a constant independent of $\alpha$. Inequalities (30) and (31) yield

$$
\left\langle H \Phi_{2}, \Phi_{2}\right\rangle-\epsilon^{-1}\left\langle\frac{\alpha}{|x|} \Phi_{2}, \Phi_{2}\right\rangle \geq\left(\Sigma_{0}-e_{0}+\frac{7}{8}\left(e_{0}-e_{1}\right)\right)\left\|\Phi_{2}\right\|^{2} .
$$

- For $T(p)$ being the self-energy operator with fixed total momentum $p \in \mathbb{R}^{3}$ defined in (7), we have from $[8$, Theorem 3.1 (B)]

$$
\left|\inf \sigma(T(p))-p^{2}-\inf \sigma(T(0))\right| \leq c_{0} \alpha p^{2} .
$$

Therefore,

$$
T(p) \geq\left(1-o_{\alpha}(1)\right) p^{2}+\Sigma_{0}
$$


Case 1. If $\left\|\Phi_{2}\right\|^{2} \geq 8\left\|\Phi_{1}\right\|^{2}$, we first do the following estimate, using (33):

$$
\begin{aligned}
& \left\langle H \Phi_{1}, \Phi_{1}\right\rangle-\epsilon\left\langle\frac{\alpha}{|x|} \Phi_{1}, \Phi_{1}\right\rangle \\
& \geq\left(1-o_{\alpha}(1)\right)\left(P^{2} \Phi_{1}, \Phi_{1}\right\rangle-\left\langle(1+\epsilon) \frac{\alpha}{|x|} \Phi_{1}, \Phi_{1}\right\rangle+\Sigma_{0}\left\|\Phi_{1}\right\|^{2} \\
& \geq\left(\Sigma_{0}-(1+\mathcal{O}(\alpha)+\mathcal{O}(\epsilon)) e_{0}\right)\left\|\Phi_{1}\right\|^{2} .
\end{aligned}
$$

Therefore, together with $\left\|\Phi_{2}\right\|^{2} \geq 8\left\|\Phi_{1}\right\|^{2}$ and (32), for $\alpha$ and $\epsilon$ small enough this implies

$$
\langle H \Phi, \Phi\rangle \geq\left(\Sigma_{0}-e_{0}\right)\|\Phi\|^{2}+\frac{3}{4}\left(e_{0}-e_{1}\right)\|\Phi\|^{2} .
$$

Case 2. If $\left\|\Phi_{2}\right\|^{2}<8\left\|\Phi_{1}\right\|^{2}$, we write the estimate

$$
\begin{aligned}
& \left\langle H \Phi_{1}, \Phi_{1}\right\rangle-\epsilon\left\langle\frac{\alpha}{|x|} \Phi_{1}, \Phi_{1}\right\rangle \\
& \geq\left(1-o_{\alpha}(1)\right)\left(P^{2} \Phi_{1}, \Phi_{1}\right\rangle-\left\langle(1+\epsilon) \frac{\alpha}{|x|} \Phi_{1}, \Phi_{1}\right\rangle+\Sigma_{0}\left\|\Phi_{1}\right\|^{2} \\
& \geq\left(1+o_{\alpha}(1)+\mathcal{O}(\epsilon)\right)\left(-e_{0} \sum_{k=0}^{\infty}\left\|\left\langle\pi^{k} \Phi_{1}, u_{\alpha}\right\rangle_{L^{2}\left(\mathbb{R}^{3}, \mathrm{~d} x\right)}\right\|^{2}-e_{1}\left(\left\|\Phi_{1}\right\|^{2}\right.\right. \\
& \left.\left.\quad-\sum_{k=0}^{\infty}\left\|\left\langle\pi^{k} \Phi_{1}, u_{\alpha}\right\rangle_{L^{2}\left(\mathbb{R}^{3}, \mathrm{~d} x\right)}\right\|^{2}\right)\right)+\Sigma_{0}\left\|\Phi_{1}\right\|^{2} .
\end{aligned}
$$

Now, by orthogonality of $\Phi$ and $u_{\alpha}$ in the sense that for all $k,\left\langle\pi^{k} \Phi, u_{\alpha}\right\rangle_{L^{2}\left(\mathbb{R}^{3}, \mathrm{~d} x\right)}$ $=0$, we get

$$
\begin{aligned}
& \sum_{k=0}^{\infty}\left\|\left\langle u_{\alpha}, \pi^{k} \Phi_{1}\right\rangle_{L^{2}\left(\mathbb{R}^{3}, \mathrm{~d} x\right)}\right\|^{2}=\sum_{k=0}^{\infty}\left\|\left\langle u_{\alpha}, \pi^{k} \Phi_{2}\right\rangle_{L^{2}\left(\mathbb{R}^{3}, \mathrm{~d} x\right)}\right\|^{2} \\
& \quad \leq\left\|\Phi_{2}\right\|^{2}\left\|\chi\left(|P| \geq \frac{p_{c}}{2}\right) u_{\alpha}\right\|^{2} \leq 8\left\|\Phi_{1}\right\|^{2}\left\|\chi\left(|P| \geq \frac{p_{c}}{2}\right) u_{\alpha}\right\|^{2} \rightarrow_{\alpha \rightarrow 0} 0
\end{aligned}
$$

Thus, for $\alpha$ and $\epsilon$ small enough, (36), (37) and (32) imply also (35) in that case.

- Let $\widetilde{c}=\max \left\{\delta,\left|c_{0}\right| \alpha^{2}\right\}$.

If $\left\langle H_{f} \Phi, \Phi\right\rangle<8 \widetilde{c}\|\Phi\|^{2}$, (16) follows from (35) with $\nu=\delta /(16 \widetilde{c})$.

If $\left\langle H_{f} \Phi, \Phi\right\rangle \geq 8 \widetilde{c}\|\Phi\|^{2}$, using Lemma 4.1, we obtain

$$
\begin{aligned}
\langle H \Phi, \Phi\rangle & \geq \frac{1}{2}\left\langle H_{f} \Phi, \Phi\right\rangle-c_{0} \alpha^{2}\|\Phi\|^{2} \geq \frac{1}{4}\left\langle H_{f} \Phi, \Phi\right\rangle+\widetilde{c}\|\Phi\|^{2} \\
& \geq \frac{1}{4}\left\langle H_{f} \Phi, \Phi\right\rangle+\delta\|\Phi\|^{2}+\left(\Sigma_{0}-e_{0}\right)\|\Phi\|^{2}
\end{aligned}
$$

since $\Sigma_{0}-e_{0} \leq 0$, which yields (16) with $\nu=\frac{1}{4}$.

This concludes the proof of (16). 


\section{Estimate of the Binding Energy up to $\alpha^{3}$ Term}

Definition 5.1. Let $u_{\alpha}$ be the normalized ground state of the Schrödinger operator $h_{\alpha}$, as defined in (15).

We define the projection $\Psi^{u_{\alpha}} \in \mathfrak{F}$ of the normalized ground state $\Psi^{\mathrm{GS}}$ of $H$, onto $u_{\alpha}$ as follows:

$$
\Psi^{\mathrm{GS}}=u_{\alpha} \Psi^{u_{\alpha}}+\Psi^{\perp}
$$

where for all $k \geq 0$,

$$
\left\langle u_{\alpha}, \pi^{k} \Psi^{\perp}\right\rangle_{L^{2}\left(\mathbb{R}^{3}, \mathrm{~d} x\right)}=0 .
$$

Remark 5.1. The definition implies that for all $m$

$$
\begin{aligned}
& \left(\pi^{m} \Psi^{u_{\alpha}}\right)\left(k_{1}, \lambda_{1} ; k_{2}, \lambda_{2} ; \ldots ; k_{m}, \lambda_{m}\right) \\
& \quad=\int_{\mathbb{R}^{3}}\left(\pi^{m} \Psi^{\mathrm{GS}}\right)\left(y ; k_{1}, \lambda_{1} ; \ldots ; k_{m}, \lambda_{m}\right) \overline{u_{\alpha}(y)} \mathrm{d} y .
\end{aligned}
$$

Definition 5.2. Let

$$
\begin{aligned}
& \Phi_{*}^{2}:=-\left(H_{f}+P_{f}^{2}\right)^{-1} A^{+} \cdot A^{+} \Omega_{f} \\
& \Phi_{*}^{3}:=-\left(H_{f}+P_{f}^{2}\right)^{-1} P_{f} \cdot A^{+} \Phi_{*}^{2} \\
& \Phi_{*}^{1}:=-\left(H_{f}+P_{f}^{2}\right)^{-1} P_{f} \cdot A^{-} \Phi_{*}^{2}
\end{aligned}
$$

where evidently, the state $\Phi_{*}^{i}$ contains $i$ photons.

Definition 5.3. On $\mathfrak{F}$, we define the positive bilinear form

$$
\langle v, w\rangle_{*}:=\left\langle v,\left(H_{f}+P_{f}^{2}\right) w\right\rangle,
$$

and its associated semi-norm $\|v\|_{*}=\langle v, v\rangle_{*}^{1 / 2}$.

We will also use the same notation for this bilinear forms on $\mathfrak{F}_{n}, \mathfrak{H}$ and $\mathfrak{H}_{n}$.

Similarly, we define the bilinear form $\langle., .\rangle_{\sharp}$ on $\mathfrak{H}$ as

$$
\langle u, v\rangle_{\sharp}:=\left\langle u,\left(H_{f}+P_{f}^{2}+h_{\alpha}+e_{0}\right) v\right\rangle
$$

and its associated semi-norm $\|v\|_{\sharp}=\langle v, v\rangle_{\sharp}^{1 / 2}$.

Definition 5.4. Let

$$
\Phi_{*}^{u_{\alpha}}:=2 \alpha^{\frac{1}{2}} \nabla u_{\alpha} \cdot\left(H_{f}+P_{f}^{2}\right)^{-1} A^{+} \Omega_{f},
$$

and

$$
\Phi_{\sharp}^{u_{\alpha}}:=2 \alpha^{\frac{1}{2}}\left(H_{f}+P_{f}^{2}+h_{\alpha}+e_{0}\right)^{-1} A^{+} \cdot \nabla u_{\alpha} \Omega_{f} .
$$

Remark 5.2. The function $\Phi_{*}^{u_{\alpha}}$ is not a vector in the Hilbert space $\mathfrak{H}$ because of the infrared singularity of the photon form factor. However, in the rest of the paper, we only used the vectors $H_{f}^{\gamma} \Phi_{*}^{u_{\alpha}}$ or $P_{f}^{\gamma} \Phi_{*}^{u_{\alpha}}$, with some $\gamma>0$, which are always well defined. In particular, all expressions involving $\left(H_{f}+P_{f}^{2}\right)^{-1}$ are always well-defined in the sequel.

The next theorem gives an upper bound on the binding energy up to the term $\alpha^{3}$ with an error term $\mathcal{O}\left(\alpha^{4}\right)$. 
Theorem 5.1 (Lower bound on the binding energy). We have

$$
\Sigma \leq \Sigma_{0}-e_{0}-\left\|\Phi_{\sharp}^{u_{\alpha}}\right\|_{\sharp}^{2}+\mathcal{O}\left(\alpha^{4}\right)
$$

Proof. Using the trial function in $\mathfrak{H}$

$$
\Phi^{\text {trial }}:=u_{\alpha}\left(\Omega_{f}+2 \alpha^{\frac{3}{2}} \Phi_{*}^{1}+\alpha \Phi_{*}^{2}+2 \alpha^{\frac{3}{2}} \Phi_{*}^{3}\right)+\Phi_{\sharp}^{u_{\alpha}},
$$

and from [7, Theorem 3.1] which states

$$
\Sigma_{0}=-\alpha^{2}\left\|\Phi_{*}^{2}\right\|_{*}^{2}+\alpha^{3}\left(2\left\|A^{-} \Phi_{*}^{2}\right\|^{2}-4\left\|\Phi_{*}^{1}\right\|_{*}^{2}-4\left\|\Phi_{*}^{3}\right\|_{*}^{2}\right)+\mathcal{O}\left(\alpha^{4}\right),
$$

the result follows straightforwardly.

We decompose the function $\Psi^{u_{\alpha}}$ defined in Definition 5.1 as follows:

Definition 5.5. Let $\eta_{1}, \eta_{2}, \eta_{3}$ and $\Delta_{*}^{u_{\alpha}}$ be defined by

$$
\Psi^{u_{\alpha}}=: \pi^{0} \Psi^{u_{\alpha}}+2 \eta_{1} \alpha^{\frac{3}{2}} \Phi_{*}^{1}+\eta_{2} \alpha \Phi_{*}^{2}+2 \eta_{3} \alpha^{\frac{3}{2}} \Phi_{*}^{3}+\Delta_{*}^{u_{\alpha}},
$$

with the conditions $\pi^{0} \Delta_{*}^{u_{\alpha}}=0$ and $\left\langle\Phi_{*}^{i}, \pi^{i} \Delta_{*}^{u_{\alpha}}\right\rangle_{*}=0(\mathrm{i}=1,2,3)$, where $\Phi_{*}^{1}$, $\Phi_{*}^{2}, \Phi_{*}^{3}$ are given in Definition 5.2.

We further decompose $\Psi^{\perp}$ into two parts.

Definition 5.6. Let

$$
\Psi^{\perp}=: \kappa_{1} \Phi_{\sharp}^{u_{\alpha}}+\Delta_{\sharp}^{\perp},
$$

be defined by $\left\langle\Phi_{\sharp}^{u_{\alpha}}, \pi^{1} \Delta_{\sharp}^{\perp}\right\rangle_{\sharp}=0$.

The following theorem provides an upper bound of the binding energy with an error term of the order $\mathcal{O}\left(\alpha^{4}\right)$. Together with Theorem 5.1, it establishes an estimate up to the order $\alpha^{3}$ with an error term $\mathcal{O}\left(\alpha^{4}\right)$.

Theorem 5.2 (Upper bound on the binding energy).

(1) Let $\Sigma=\inf \sigma(H)$. Then

$$
\begin{aligned}
\Sigma & \geq \Sigma_{0}-e_{0}-\left\|\Phi_{*}^{u_{\alpha}}\right\|_{*}^{2}+\mathcal{O}\left(\alpha^{4}\right), \\
\Sigma_{0} & =-\alpha^{2}\left\|\Phi_{*}^{2}\right\|_{*}^{2}+\alpha^{3}\left(2\left\|A^{-} \Phi_{*}^{2}\right\|^{2}-4\left\|\Phi_{*}^{1}\right\|_{*}^{2}-4\left\|\Phi_{*}^{3}\right\|_{*}^{2}\right)+\mathcal{O}\left(\alpha^{4}\right),
\end{aligned}
$$

and $\Phi_{*}^{u_{\alpha}}$ defined by (41).

(2) For the components $\Delta_{\sharp}^{\perp}, \Psi^{u_{\alpha}}, \Delta_{*}^{u_{\alpha}}$ of the ground state $\Psi^{G S}$, and the coefficients $\eta_{1}, \eta_{2}, \eta_{3}$ and $\kappa_{1}$ defined in Definitions 5.1-5.5, holds

$$
\begin{gathered}
\left\|\Delta_{\sharp}^{\perp}\right\|^{2}=\mathcal{O}\left(\alpha^{\frac{33}{16}}\right), \quad\left\|H_{f}^{\frac{1}{2}} \Delta_{\sharp}^{\perp}\right\|^{2}=\mathcal{O}\left(\alpha^{4}\right), \quad\left\|\left(P-P_{f}\right) \Delta_{\sharp}^{\perp}\right\|^{2}=\mathcal{O}\left(\alpha^{4}\right), \\
\left|\pi^{0} \Psi^{u_{\alpha}}\right|^{2} \geq 1-c \alpha^{2}, \\
\left\|\Delta_{*}^{u_{\alpha}}\right\|_{*}^{2}=\mathcal{O}\left(\alpha^{4}\right), \quad\left\|\Delta_{*}^{u_{\alpha}}\right\|^{2}=\mathcal{O}\left(\alpha^{\frac{33}{16}}\right), \\
\left|\eta_{1,3}-1\right|^{2} \leq c \alpha, \quad\left|\eta_{2}-1\right|^{2} \leq c \alpha^{2}, \quad\left|\kappa_{1}-1\right|^{2} \leq c \alpha .
\end{gathered}
$$

To prove this theorem, we will compute $\left\langle H \Psi^{\mathrm{GS}}, \Psi^{\mathrm{GS}}\right\rangle$ according to the decomposition of $\Psi^{\mathrm{GS}}$ introduced in Definitions 5.1 to 5.6. Using 


$$
H=\left(P^{2}-\frac{\alpha}{|x|}\right)+:\left(P_{f}+\sqrt{\alpha} A(0)\right)^{2}:+H_{f}-2 \operatorname{Re} P \cdot\left(P_{f}+\sqrt{\alpha} A(0)\right),
$$

and due to the orthogonality of $u_{\alpha}$ and $\Psi^{\perp}$, we obtain

$$
\begin{aligned}
\left\langle H \Psi^{\mathrm{GS}}, \Psi^{\mathrm{GS}}\right\rangle= & \left\langle H u_{\alpha} \Psi^{u_{\alpha}}, u_{\alpha} \Psi^{u_{\alpha}}\right\rangle+\left\langle H \Psi^{\perp}, \Psi^{\perp}\right\rangle \\
& -4 \operatorname{Re}\left\langle P .\left(P_{f}+\sqrt{\alpha} A(0)\right) u_{\alpha} \Psi^{u_{\alpha}}, \Psi^{\perp}\right\rangle .
\end{aligned}
$$

We will estimate separately each term in (49) in Sects. 7.1-7.3. These estimates will be used to establish in Sect. 7.4 the proof of Theorem 5.2.

\section{Estimate of the Binding Energy up to $o\left(\alpha^{5} \log \alpha^{-1}\right)$ Term}

We develop here the proof of the difficult part in Theorem 2.1 which is the upper bound in (8), and which is stated in Theorem 6.1 below for convenience.

Some technical aspects of this proof are detailed in Sect. 8 and Appendices $\mathrm{A}$ and $\mathrm{B}$.

Theorem 6.1 (Upper bound up to the order $\alpha^{5} \log \alpha^{-1}$ for the binding energy). For $\alpha$ small enough, we have

$$
\Sigma_{0}-\Sigma \geq \frac{1}{4} \alpha^{2}+e^{(1)} \alpha^{3}+e^{(2)} \alpha^{4}+e^{(3)} \alpha^{5} \log \alpha^{-1}+o\left(\alpha^{5} \log \alpha^{-1}\right),
$$

where $e^{(1)}, e^{(2)}$ and $e^{(3)}$ are defined in Theorem 2.1 .

In order to prove this result, we need to refine the splitting of the function $\Psi^{\perp}$ orthogonal to $u_{\alpha} \Psi^{u_{\alpha}}$ defined in Definition 5.1. Therefore, we consider the following decomposition:

Definition 6.1. (1) Pick

$$
\kappa_{2}= \begin{cases}\alpha^{-1} \frac{\left\langle\Psi^{\perp}, \Phi_{*}^{2} \pi^{0} \Psi^{\perp}\right\rangle_{\sharp}}{\left\langle\Phi_{*}^{2} \pi^{0} \Psi^{\perp}, \Phi_{*}^{2} \pi^{0} \Psi^{\perp}\right\rangle_{\sharp}} & \text { if }\left\|\pi^{0} \Psi^{\perp}\right\|>\alpha^{\frac{3}{2}}, \\ 0 & \text { if }\left\|\pi^{0} \Psi^{\perp}\right\| \leq \alpha^{\frac{3}{2}} .\end{cases}
$$

(2) We split $\Psi^{\perp}$ into $\Psi_{1}^{\perp}$ and $\Psi_{2}^{\perp}$ as follows: $\forall n \geq 0, \pi^{n} \Psi^{\perp}=\pi^{n} \Psi_{1}^{\perp}+\pi^{n} \Psi_{2}^{\perp}$ and

for $n=0$,

$$
\pi^{0} \Psi_{1}^{\perp}=\pi^{0} \Psi^{\perp} \quad \text { and } \quad \pi^{0} \Psi_{2}^{\perp}=0
$$

for $n=1$,

$$
\pi^{1} \Psi_{1}^{\perp}=\kappa_{1} \Phi_{\sharp}^{u_{\alpha}} \quad \text { and } \quad\left\langle\pi^{1} \Psi_{2}^{\perp}, \Phi_{\sharp}^{u_{\alpha}}\right\rangle_{\sharp}=0,
$$

for $n=2$,

$$
\begin{aligned}
\pi^{2} \Psi_{1}^{\perp}= & \alpha \kappa_{2} \Phi_{*}^{2} \pi^{0} \Psi_{1}^{\perp}+\sum_{i=1}^{3} \alpha \kappa_{2, i}\left(H_{f}+P_{f}^{2}\right)^{-1} W_{i} \frac{\partial u_{\alpha}}{\partial x_{i}}, \\
& \text { with } W_{i}=P_{f}^{i} \Phi_{*}^{2}-2 A^{+} . P_{f}\left(H_{f}+P_{f}^{2}\right)^{-1}\left(A^{+}\right)^{i} \Omega_{f}, \\
\pi^{2} \Psi_{2}^{\perp}= & \pi^{2} \Psi^{\perp}-\pi^{2} \Psi_{1}^{\perp}, \quad \text { with }\left\langle\pi^{2} \Psi_{2}^{\perp},\left(H_{f}+P_{f}^{2}\right)^{-1} W_{i} \frac{\partial u_{\alpha}}{\partial x_{i}}\right\rangle_{\sharp}=0 \quad(i=1,2,3), \\
& \text { and }\left\langle\pi^{2} \Psi_{2}^{\perp}, \Phi_{*}^{2} \pi^{0} \Psi_{1}^{\perp}\right\rangle_{\sharp}=0,
\end{aligned}
$$


for $n=3$,

$$
\begin{aligned}
& \pi^{3} \Psi_{1}^{\perp}=\alpha \kappa_{3}\left(H_{f}+P_{f}^{2}\right)^{-1} A^{+} \cdot A^{+} \Phi_{\sharp}^{u_{\alpha}}, \\
& \pi^{3} \Psi_{2}^{\perp}=\pi^{3} \Psi^{\perp}-\pi^{3} \Psi_{1}^{\perp},\left\langle\pi^{3} \Psi_{2}^{\perp},\left(H_{f}+P_{f}^{2}\right)^{-1} A^{+} \cdot A^{+} \Phi_{\sharp}^{u_{\alpha}}\right\rangle_{\sharp}=0,
\end{aligned}
$$

and for $n \geq 4$,

$$
\pi^{n} \Psi_{1}^{\perp}=0 \quad \text { and } \quad \pi^{n} \Psi_{2}^{\perp}=\pi^{n} \Psi^{\perp} .
$$

The next step consists in establishing, in the next lemma, some a priori estimates concerning the function $\Psi^{\perp}$ that give additional information to those obtained in (45) of Theorem 5.2.

Lemma 6.1. The following estimates hold:

$$
\begin{aligned}
\kappa_{1} & =1+\mathcal{O}\left(\alpha^{\frac{1}{2}}\right), \\
\left|\kappa_{2}\right|\left\|\pi^{0} \Psi_{1}^{\perp}\right\| & =\mathcal{O}(\alpha), \\
\kappa_{2, i} & =\mathcal{O}(1), \quad(i=1,2,3), \\
\left\|\pi^{0} \Psi_{1}^{\perp}\right\| & =\mathcal{O}(\alpha), \\
\left\|P \pi^{0} \Psi_{1}^{\perp}\right\| & =\mathcal{O}\left(\alpha^{2}\right) .
\end{aligned}
$$

Proof. To derive these estimates, we use Theorem 5.2.

The first equality is a consequence of (48).

To derive the next two estimates, we first notice that (45) yields

$$
\begin{aligned}
\left\|P \pi^{2} \Delta_{\sharp}^{\perp}\right\|^{2} & \leq 2\left\|\left(P-P_{f}\right) \Delta_{\sharp}^{\perp}\right\|^{2}+2\left\|P_{f} \pi^{2} \Delta_{\sharp}^{\perp}\right\|^{2} \\
& \leq 2\left\|\left(P-P_{f}\right) \Delta_{\sharp}^{\perp}\right\|^{2}+2 c\left\|H_{f}^{\frac{1}{2}} \pi^{2} \Delta_{\sharp}^{\perp}\right\|^{2}=\mathcal{O}\left(\alpha^{4}\right),
\end{aligned}
$$

therefore, using again (45), we obtain

$$
\left\|\left(h_{\alpha}+e_{0}\right)^{\frac{1}{2}} \pi^{2} \Delta_{\sharp}^{\perp}\right\|^{2} \leq\left\|P \pi^{2} \Delta_{\sharp}^{\perp}\right\|^{2}+e_{0}\left\|\pi^{2} \Delta_{\sharp}^{\perp}\right\|^{2}=\mathcal{O}\left(\alpha^{4}\right),
$$

and thus, using from (45) that $\left\|H_{f}^{\frac{1}{2}} \pi^{2} \Delta_{\sharp}^{\perp}\right\|^{2}=\mathcal{O}\left(\alpha^{4}\right)$, we get

$$
\left\|\pi^{2} \Delta_{\sharp}^{\perp}\right\|_{\sharp}^{2}=\left\|\pi^{2} \Psi^{\perp}\right\|_{\sharp}^{2}=\mathcal{O}\left(\alpha^{4}\right) \text {. }
$$

We then write, using (51) and the $\langle\cdot, \cdot\rangle_{\sharp}$-orthogonality of $\pi^{2} \Psi_{1}^{\perp}$ and $\pi^{2} \Psi_{2}^{\perp}$,

$$
\left\|\pi^{2} \Psi_{1}^{\perp}\right\|_{\sharp}^{2} \leq\left\|\pi^{2} \Psi^{\perp}\right\|_{\sharp}^{2}=\mathcal{O}\left(\alpha^{4}\right) .
$$

Since $\left\|\pi^{2} \Psi_{1}^{\perp}\right\|_{\sharp} \leq\left\|\pi^{2} \Psi_{1}^{\perp}\right\|_{*}$, and using (206) of Lemma C.2, we obtain

$$
\begin{aligned}
\mathcal{O}\left(\alpha^{4}\right) & =\left\|\pi^{2} \Psi_{1}^{\perp}\right\|_{*}^{2}=\left\|\alpha \kappa_{2} \Phi_{*}^{2} \pi^{0} \Psi_{1}^{\perp}\right\|_{*}^{2}+\left\|\alpha \sum_{i} \kappa_{2, i}\left(H_{f}+P_{f}^{2}\right)^{-1} W_{i} \frac{\partial u_{\alpha}}{\partial x_{i}}\right\|_{*}^{2} \\
& =\alpha^{2}\left\|\Phi_{*}^{2}\right\|_{*}^{2}\left|\kappa_{2}\right|^{2}\left\|\pi^{0} \Psi_{1}^{\perp}\right\|^{2}+\frac{\alpha^{2}}{3}\left\|\nabla u_{\alpha}\right\|^{2} \sum_{i}\left|\kappa_{2, i}\right|^{2}\left\|\left(H_{f}+P_{f}^{2}\right)^{-1} W_{i}\right\|_{*}^{2} .
\end{aligned}
$$

which gives

$$
\kappa_{2, i}=\mathcal{O}(1) \quad \text { and } \quad\left|\kappa_{2}\right|\left\|\pi^{0} \Psi_{1}^{\perp}\right\|=\mathcal{O}(\alpha) .
$$

The last two estimates are consequences of (45). 
Eventually, to derive the lower bound on the quadratic form of $\left\langle H\left(u_{\alpha} \Psi^{u_{\alpha}}+\Psi^{\perp}\right), u_{\alpha} \Psi^{u_{\alpha}}+\Psi^{\perp}\right\rangle$, yielding the upper bound (50) of Theorem 6.1, we will follow the same strategy as in Sect. 5, the only difference being that now we have better a priori estimates on $\Psi^{u_{\alpha}}$ and $\Psi^{\perp}$.

The two main results to achieve this are stated below, with the computation of the contribution to the ground-state energy of the cross term (Proposition 6.1) and of the direct term $\left\langle H \Psi^{\perp}, \Psi^{\perp}\right\rangle$ (Proposition 6.2). Equipped with this two propositions, and using Theorem 5.2 and Lemma 6.1, the proof of Theorem 6.1 is only a straightforward computation which is detailed in Sect. 8 .

Proposition 6.1. We have

$$
\begin{aligned}
& 2 \operatorname{Re}\left\langle H \Psi^{\perp}, u_{\alpha} \Psi^{u_{\alpha}}\right\rangle \geq-\frac{1}{3} \alpha^{4} \operatorname{Re} \sum_{i=1}^{3} \overline{\kappa_{2, i}}\left\langle\left(H_{f}+P_{f}^{2}\right)^{-1} P_{f}^{i} \Phi_{*}^{2}, W_{i}\right\rangle \\
& -4 \alpha \operatorname{Re}\left\langle\nabla u_{\alpha} \cdot P_{f} \Phi_{*}^{2}, \pi^{2} \Psi_{2}^{\perp}\right\rangle-2 \operatorname{Re} \overline{\kappa_{1}} \pi^{0} \Psi^{u_{\alpha}}\left\|\Phi_{\sharp}^{u_{\alpha}}\right\|_{\sharp}^{2} \\
& \quad-\frac{2}{3} \alpha^{4} \operatorname{Re} \sum_{i=1}^{3}\left\langle\left(H_{f}+P_{f}^{2}\right)^{-\frac{1}{2}}\left(A^{-}\right)^{i} \Phi_{*}^{2},\left(H_{f}+P_{f}^{2}\right)^{-\frac{1}{2}}\left(A^{+}\right)^{i} \Omega_{f}\right\rangle \\
& -\epsilon \alpha^{2}\left\|\left(\Psi_{1}^{\perp}\right)^{a}\right\|^{2}-\frac{5}{8} M\left[\Psi_{2}^{\perp}\right]-\epsilon \alpha^{5} \log \alpha^{-1}-\epsilon \alpha^{5}\left|\kappa_{3}\right|^{2}-\left|\kappa_{1}-1\right| c \alpha^{4}+\mathcal{O}\left(\alpha^{5}\right) .
\end{aligned}
$$

The proof of this Proposition is detailed in Appendix A

\section{Proposition 6.2.}

$$
\begin{aligned}
& \left\langle H \Psi^{\perp}, \Psi^{\perp}\right\rangle \geq\left(\Sigma_{0}-e_{0}\right)\left\|\Psi^{\perp}\right\|^{2}-4 \alpha\left\|\left(h_{\alpha}+e_{0}\right)^{-\frac{1}{2}} Q_{\alpha}^{\perp} P A^{-} \Phi_{*}^{u_{\alpha}}\right\|^{2} \\
& +\left|\kappa_{1}\right|^{2}\left\|\Phi_{\sharp}^{u_{\alpha}}\right\|_{\sharp}^{2}+2 \alpha\left\|A^{-} \Phi_{*}^{u_{\alpha}}\right\|^{2}+\frac{\alpha^{4}}{12} \sum_{i=1}^{3}\left|\kappa_{2, i}\right|^{2}\left\|\left(H_{f}+P_{f}^{2}\right)^{-1} W_{i}\right\|_{*}^{2} \\
& \quad+\frac{2}{3} \alpha^{4} \operatorname{Re} \sum_{i=1}^{3} \kappa_{2, i}\left\langle P_{f} \cdot A^{-}\left(H_{f}+P_{f}^{2}\right)^{-1} W_{i},\left(H_{f}+P_{f}^{2}\right)^{-1}\left(A^{+}\right)^{i} \Omega_{f}\right\rangle \\
& +4 \alpha^{\frac{1}{2}} \operatorname{Re}\left\langle\pi^{2} \Psi_{2}^{\perp}, A^{+} . P_{f} \Phi_{*}^{u_{\alpha}}\right\rangle+M_{1}\left[\Psi^{\perp}\right],
\end{aligned}
$$

where

$$
\begin{aligned}
M_{1}\left[\Psi^{\perp}\right]= & \left(1-c_{0} \alpha\right)\left\|\left(h_{\alpha}+e_{0}\right)^{\frac{1}{2}} \pi^{0}\left(\Psi_{1}^{\perp}\right)^{a}\right\|^{2}+\frac{\left|\kappa_{3}+1\right|^{2}}{2} \alpha^{2}\left\|\Phi_{*}^{2}\right\|_{*}^{2}\left\|\Phi_{\sharp}^{u_{\alpha}}\right\|^{2} \\
& -\left|\kappa_{1}-1\right| c \alpha^{4}+\frac{3}{4} M\left[\Psi_{2}^{\perp}\right]+o\left(\alpha^{5} \log \alpha^{-1}\right),
\end{aligned}
$$

and $Q_{\alpha}^{\perp}$ is the projection onto the orthogonal complement to the ground state $u_{\alpha}$ of the Schrödinger operator $h_{\alpha}=-\Delta-\frac{\alpha}{|x|}$

The proof of this Proposition is detailed in Appendix B. 


\section{Proof of Theorem $\mathbf{5 . 2}$}

We prove Theorem 5.2 by bounding the individual terms in the expression for the binding energy.

\subsection{Estimate of the Term $\left\langle H u_{\alpha} \Psi^{u_{\alpha}}, u_{\alpha} \Psi^{u_{\alpha}}\right\rangle$}

\section{Lemma 7.1.}

$$
\begin{aligned}
\left\langle H u_{\alpha}\right. & \left.\Psi^{u_{\alpha}}, u_{\alpha} \Psi^{u_{\alpha}}\right\rangle \\
\geq & -e_{0}\left\|\Psi^{u_{\alpha}}\right\|^{2}-\alpha^{2}\left\|\Phi_{*}^{2}\right\|_{*}^{2}\left\|\pi^{0} \Psi^{u_{\alpha}}\right\|^{2}+\alpha^{2}\left|\eta_{2}-\pi^{0} \Psi^{u_{\alpha}}\right|^{2}\left\|\Phi_{*}^{2}\right\|_{*}^{2} \\
& +\alpha^{3}\left|\eta_{2}\right|^{2}\left(2\left\|A^{-} \Phi_{*}^{2}\right\|^{2}-4\left\|\Phi_{*}^{1}\right\|_{*}^{2}-4\left\|\Phi_{*}^{3}\right\|_{*}^{2}\right) \\
& +4 \alpha^{3}\left(\left|\eta_{1}-\eta_{2}\right|^{2}\left\|\Phi_{*}^{1}\right\|_{*}^{2}+\left|\eta_{3}-\eta_{2}\right|^{2}\left\|\Phi_{*}^{3}\right\|_{*}^{2}\right) \\
& +c \alpha^{4} \log \alpha^{-1}\left(\left|\eta_{1}\right|^{2}+\left|\eta_{2}\right|^{2}+\left|\eta_{3}\right|^{2}+\left\|\pi^{0} \Psi^{u_{\alpha}}\right\|^{2}\right)+\frac{1}{2}\left\|\Delta_{*}^{u_{\alpha}}\right\|_{*}^{2} .
\end{aligned}
$$

Proof. The proof is a trivial modification of the one given for the lower bound in [7, Theorem 3.1]. The only modification is that we have a slightly weaker estimate in Lemma 3.1 on the photon number for the ground state. This is accounted for by replacing the term of order $\alpha^{4}$ in [7, Theorem 3.1] by a term of order $\alpha^{4} \log \alpha^{-1}$. In addition, we need to use the equality $\left\langle P .\left(P_{f}+\right.\right.$ $\left.\sqrt{\alpha} A(0)) u_{\alpha} \Psi^{u_{\alpha}}, u_{\alpha} \Psi^{u_{\alpha}}\right\rangle=0$, due to the symmetry of $u_{\alpha}$.

\subsection{Estimates for the Cross Term -4Re $\left\langle P .\left(P_{f}+\sqrt{\alpha} A(0)\right) u_{\alpha} \Psi^{u_{\alpha}}, \Psi^{\perp}\right\rangle$}

Lemma $7.2\left(-4 \operatorname{Re}\left\langle P . P_{f} u_{\alpha} \Psi^{u_{\alpha}}, \Psi^{\perp}\right\rangle\right.$ term).

$$
\begin{aligned}
& -4 \operatorname{Re}\left\langle P . P_{f} u_{\alpha} \Psi^{u_{\alpha}}, \Psi^{\perp}\right\rangle \\
& \quad \geq-c \alpha^{4}\left(\left|\eta_{1}\right|^{2}+\left|\eta_{2}\right|^{2}+\left|\eta_{3}\right|^{2}\right)-\epsilon\left\|H_{f}^{\frac{1}{2}} \Delta_{\sharp}^{\perp}\right\|^{2}-c \alpha\left\|H_{f}^{\frac{1}{2}} \Delta_{*}^{u_{\alpha}}\right\|^{2} .
\end{aligned}
$$

Proof. - For $n=1$ photon,

$$
\left\langle P . P_{f} \pi^{1} u_{\alpha} \Psi^{u_{\alpha}}, \Psi^{\perp}\right\rangle=\left\langle P . P_{f}\left(\eta_{1} \alpha^{\frac{3}{2}} \Phi_{*}^{1}+\pi^{1} \Delta_{*}^{u_{\alpha}}\right) u_{\alpha}, \kappa_{1} \Phi_{\sharp}^{u_{\alpha}}+\pi^{1} \Delta_{\sharp}^{\perp}\right\rangle .
$$

Obviously

$$
\begin{aligned}
& \left|\left\langle P . P_{f}\left(\eta_{1} \alpha^{\frac{3}{2}} \Phi_{*}^{1}+\pi^{1} \Delta_{*}^{u_{\alpha}}\right) u_{\alpha}, \pi^{1} \Delta_{\sharp}^{\perp}\right\rangle\right| \\
& \quad \leq \epsilon\left\|H_{f}^{\frac{1}{2}} \pi^{1} \Delta_{\sharp}^{\perp}\right\|^{2}+c \alpha^{5}\left|\eta_{1}\right|^{2}+c \alpha^{2}\left\|H_{f}^{\frac{1}{2}} \pi^{1} \Delta_{*}^{u_{\alpha}}\right\|^{2} .
\end{aligned}
$$

Due to Lemma C.5 holds $\left\|H_{f}^{\frac{1}{2}}\left(\Phi_{\sharp}^{u_{\alpha}}-\Phi_{*}^{u_{\alpha}}\right)\right\|^{2}=\mathcal{O}\left(\alpha^{5}\right)$, which implies

$$
\begin{aligned}
& \left|\left\langle P . P_{f}\left(\eta_{1} \alpha^{\frac{3}{2}} \Phi_{*}^{1}+\pi^{1} \Delta_{*}^{u_{\alpha}}\right) u_{\alpha}, \kappa_{1} \Phi_{\sharp}^{u_{\alpha}}\right\rangle\right| \\
& \leq\left|\kappa_{1}\right|^{2} c \alpha^{5}+c\left|\eta_{1}\right|^{2} \alpha^{5}+c \alpha^{2}\left\|H_{f}^{\frac{1}{2}} \pi^{1} \Delta_{*}^{u_{\alpha}}\right\|^{2} \\
& \quad+\left|\left\langle P . P_{f}\left(\eta_{1} \alpha^{\frac{3}{2}} \Phi_{*}^{1}+\pi^{1} \Delta_{*}^{u_{\alpha}}\right) u_{\alpha}, \kappa_{1} \Phi_{*}^{u_{\alpha}}\right\rangle\right| .
\end{aligned}
$$


For the last term on the right hand side of (59), due to the orthogonality of $\frac{\partial u_{\alpha}}{\partial x_{i}}$ and $\frac{\partial u_{\alpha}}{\partial x_{j}}, i \neq j$, and the equality $\left\|\frac{\partial u_{\alpha}}{\partial x_{i}}\right\|=\left\|\frac{\partial u_{\alpha}}{\partial x_{j}}\right\|$, holds

$$
\begin{aligned}
& \left|\left\langle P . P_{f}\left(\eta_{1} \alpha^{\frac{3}{2}} \Phi_{*}^{1}+\pi^{1} \Delta_{*}^{u_{\alpha}}\right) u_{\alpha}, \kappa_{1} \Phi_{*}^{u_{\alpha}}\right\rangle\right| \\
& =\sum_{i=1}^{3}\left\|\frac{\partial u_{\alpha}}{\partial x_{i}}\right\|^{2}\left|\left\langle\eta_{1} \alpha^{\frac{3}{2}} \Phi_{*}^{1}+\pi^{1} \Delta_{*}^{u_{\alpha}}, \kappa_{1} P_{f}^{i}\left(A^{+} \Omega_{f}\right)^{i}\right\rangle\right| \\
& =c\left|\left\langle\eta_{1} \alpha^{\frac{3}{2}} \Phi_{*}^{1}+\pi^{1} \Delta_{*}^{u_{\alpha}}, \kappa_{1} A^{+} . P_{f} \Omega_{f}\right\rangle\right|=0 .
\end{aligned}
$$

- For $n=2$ photons,

$$
\begin{aligned}
& \left|\left\langle P . P_{f} \pi^{2} u_{\alpha} \Psi^{u_{\alpha}}, \Psi^{\perp}\right\rangle\right|=\left|\left\langle P . P_{f}\left(\eta_{2} \alpha \Phi_{*}^{2}+\pi^{2} \Delta_{*}^{u_{\alpha}}\right) u_{\alpha}, \pi^{2} \Delta_{\sharp}^{\perp}\right\rangle\right| \\
& \quad \leq c \alpha^{4}\left|\eta_{2}\right|^{2}+\epsilon\left\|H_{f}^{\frac{1}{2}} \pi^{2} \Delta_{\sharp}^{\perp}\right\|^{2}+\alpha\left\|H_{f}^{\frac{1}{2}} \pi^{2} \Delta_{*}^{u_{\alpha}}\right\|^{2} .
\end{aligned}
$$

- For $n=3$ photons, a similar estimate yields

$$
\begin{aligned}
& \left|\left\langle P . P_{f} \pi^{3} u_{\alpha} \Psi^{u_{\alpha}}, \Psi^{\perp}\right\rangle\right|=\left|\left\langle P . P_{f}\left(\eta_{3} \alpha^{\frac{3}{2}} \Phi_{*}^{3}+\pi^{3} \Delta_{*}^{u_{\alpha}}\right) u_{\alpha}, \pi^{3} \Delta_{\sharp}^{\perp}\right\rangle\right| \\
& \leq c \alpha^{4}\left|\eta_{3}\right|^{2}+\epsilon\left\|H_{f}^{\frac{1}{2}} \pi^{3} \Delta_{\sharp}^{\perp}\right\|^{2}+\alpha\left\|H_{f}^{\frac{1}{2}} \pi^{3} \Delta_{*}^{u_{\alpha}}\right\|^{2} .
\end{aligned}
$$

- For $n \geq 4$ photons,

$$
\left|\left\langle P . P_{f} \pi^{n \geq 4} u_{\alpha} \Psi^{u_{\alpha}}, \Psi^{\perp}\right\rangle\right| \leq \epsilon\left\|H_{f}^{\frac{1}{2}} \pi^{n \geq 4} \Delta_{\sharp}^{\perp}\right\|^{2}+\alpha\left\|H_{f}^{\frac{1}{2}} \pi^{n \geq 4} \Delta_{*}^{u_{\alpha}}\right\|^{2} .
$$

Lemma $7.3\left(-4 R e\left\langle\sqrt{\alpha} P . A(0) u_{\alpha} \Psi^{u_{\alpha}}, \Psi^{\perp}\right\rangle\right.$ term).

$$
\begin{aligned}
& \left.-4 \operatorname{Re}\langle\sqrt{\alpha} P \cdot A(0)) u_{\alpha} \Psi^{u_{\alpha}}, \Psi^{\perp}\right\rangle \\
& \quad \geq-2 \operatorname{Re} \overline{\kappa_{1}} \pi^{0} \Psi^{u_{\alpha}}\left\|\Phi_{\sharp}^{u_{\alpha}}\right\|_{\sharp}^{2}-\epsilon\left\|H_{f}^{\frac{1}{2}} \Delta_{\sharp}^{\perp}\right\|^{2}-\epsilon \alpha^{2}\left\|\Delta_{\sharp}^{\perp}\right\|^{2} \\
& -c \alpha\left\|H_{f}^{\frac{1}{2}} \Delta_{*}^{u_{\alpha}}\right\|^{2}-c \alpha^{4}\left(\left|\eta_{1}\right|^{2}+\left|\eta_{2}\right|^{2}+\left|\eta_{3}\right|^{2}+\left|\kappa_{1}\right|^{2}\right)+\mathcal{O}\left(\alpha^{5} \log \alpha^{-1}\right) .
\end{aligned}
$$

Proof. We first estimate the term

$$
\operatorname{Re} \alpha^{\frac{1}{2}}\left\langle P . A^{+} u_{\alpha} \Psi^{u_{\alpha}}, \Psi^{\perp}\right\rangle=\alpha \operatorname{Re} \sum_{n=0}^{\infty}\left\langle P . A^{+} u_{\alpha} \pi^{n} \Psi^{u_{\alpha}}, \pi^{n+1} \Psi^{\perp}\right\rangle .
$$

- For $n=0$ photon, using the orthogonality $\left\langle\Phi_{\sharp}^{u_{\alpha}}, \pi^{1} \Delta_{\sharp}^{\perp}\right\rangle_{\sharp}=0$, yields

$$
\begin{aligned}
& -\operatorname{Re} \alpha^{\frac{1}{2}}\left\langle P \cdot A^{+} \pi^{0} u_{\alpha} \Psi^{u_{\alpha}}, \kappa_{1} \Phi_{\sharp}^{u_{\alpha}}+\pi^{1} \Delta_{\sharp}^{\perp}\right\rangle \\
& =-\frac{1}{2} \operatorname{Re}\left(\left(\pi^{0} \Psi^{u_{\alpha}}\right)\left\langle\Phi_{\sharp}^{u_{\alpha}}, \kappa_{1} \Phi_{\sharp}^{u_{\alpha}}+\pi^{1} \Delta_{\sharp}^{\perp}\right\rangle_{\sharp}\right) \\
& =-\frac{1}{2} \operatorname{Re}\left(\overline{\kappa_{1}} \pi^{0} \Psi^{u_{\alpha}}\left\langle\Phi_{\sharp}^{u_{\alpha}}, \Phi_{\sharp}^{u_{\alpha}}\right\rangle_{\sharp}\right) .
\end{aligned}
$$

- For $n \geq 1$ photons,

$$
\begin{gathered}
\left|\sum_{n \geq 2} \operatorname{Re} \alpha^{\frac{1}{2}}\left\langle P . A^{+} \pi^{n} u_{\alpha} \Delta_{*}^{u_{\alpha}}, \pi^{n+1} \Delta_{\sharp}^{\perp}\right\rangle\right| \leq c \alpha^{3}\left\|\Delta_{*}^{u_{\alpha}}\right\|^{2}+\epsilon\left\|H_{f}^{\frac{1}{2}} \Delta_{\sharp}^{\perp}\right\|^{2} \\
\leq \epsilon\left\|H_{f}^{\frac{1}{2}} \Delta_{\sharp}^{\perp}\right\|^{2}+c \alpha^{5}\left(\left|\eta_{1}\right|^{2}+\left|\eta_{2}\right|^{2}+\left|\eta_{3}\right|^{2}\right)+\mathcal{O}\left(\alpha^{5} \log \alpha^{-1}\right),
\end{gathered}
$$


where we used from Lemma 3.1 that $\left\|\Delta_{*}^{u_{\alpha}}\right\|^{2} \leq \mathcal{O}\left(\alpha^{2} \log \alpha^{-1}\right)+c \alpha^{3}\left(\left|\eta_{1}\right|^{2}+\right.$ $\left.\left|\eta_{3}\right|^{2}\right)+c \alpha^{2}\left|\eta_{2}\right|^{2}$. We also have

$$
\begin{gathered}
\left|\operatorname{Re} \alpha^{\frac{1}{2}}\left\langle P\left(2 \eta_{1} \alpha^{\frac{3}{2}} \Phi_{*}^{1}+\eta_{2} \alpha \Phi_{*}^{2}+2 \eta_{3} \alpha^{\frac{3}{2}} \Phi_{*}^{3}\right) u_{\alpha}, A^{-} \Delta_{\sharp}^{\perp}\right\rangle\right| \\
\leq \epsilon\left\|H_{f}^{\frac{1}{2}} \Delta_{\sharp}^{\perp}\right\|^{2}+c\left(\alpha^{6}\left|\eta_{1}\right|^{2}+\alpha^{5}\left|\eta_{2}\right|^{2}+\alpha^{6}\left|\eta_{3}\right|^{2}\right) .
\end{gathered}
$$

We next estimate the term $2 \operatorname{Re} \alpha^{\frac{1}{2}}\left\langle P . A^{-} u_{\alpha} \Psi^{u_{\alpha}}, \Psi^{\perp}\right\rangle$. We first get

$$
\left|\alpha^{\frac{1}{2}} \operatorname{Re}\left\langle P \cdot A^{-} \Delta_{*}^{u_{\alpha}} u_{\alpha}, \Delta_{\sharp}^{\perp}\right\rangle\right| \leq \epsilon \alpha^{2}\left\|\Delta_{\sharp}^{\perp}\right\|^{2}+c \alpha\left\|H_{f}^{\frac{1}{2}} \Delta_{*}^{u_{\alpha}}\right\|^{2} \cdot M
$$

Then we write

$$
\begin{aligned}
& \left|\alpha^{\frac{1}{2}} \operatorname{Re}\left\langle\left(2 \eta_{1} \alpha^{\frac{3}{2}} A^{-} \Phi_{*}^{1}+2 \eta_{3} \alpha^{\frac{3}{2}} A^{-} \Phi_{*}^{3}\right) \nabla u_{\alpha}, \Delta_{\sharp}^{\perp}\right\rangle\right| \\
& \leq \epsilon \alpha^{2}\left\|\Delta_{\sharp}^{\perp}\right\|^{2}+c \alpha^{4}\left(\left|\eta_{1}\right|^{2}+\left|\eta_{3}\right|^{2}\right) .
\end{aligned}
$$

We also have

$$
\begin{aligned}
& \left|\alpha^{\frac{1}{2}} \operatorname{Re}\left\langle\eta_{2} \alpha A^{-} \Phi_{*}^{2} \cdot \nabla u_{\alpha}, \kappa_{1} \Phi_{\sharp}^{u_{\alpha}}\right\rangle\right| \\
& \quad=\left|\alpha^{\frac{1}{2}} \operatorname{Re}\left\langle\eta_{2} \alpha H_{f}^{-\frac{1}{2}} A^{-} \Phi_{*}^{2} \cdot \nabla u_{\alpha}, H_{f}^{\frac{1}{2}} \kappa_{1} \Phi_{\sharp}^{u_{\alpha}}\right\rangle\right| \leq c \alpha^{4}\left(\left|\eta_{2}\right|^{2}+\left|\kappa_{1}\right|^{2}\right),
\end{aligned}
$$

since $H_{f}^{-\frac{1}{2}} A^{-} \Phi_{*}^{2} \in L^{2}\left(\mathbb{R}^{3}\right)$ and $\left\|H_{f}^{\frac{1}{2}} \Phi_{\sharp}^{u_{\alpha}}\right\|=\mathcal{O}\left(\alpha^{\frac{3}{2}}\right)$. Finally, we get

$$
\begin{aligned}
& \left|\alpha^{\frac{1}{2}} \operatorname{Re}\left\langle\eta_{2} \alpha A^{-} \Phi_{*}^{2} \cdot \nabla u_{\alpha}, \pi^{1} \Delta_{\sharp}^{\perp}\right\rangle\right| \\
& \quad=\left|\alpha^{\frac{1}{2}} \operatorname{Re}\left\langle\eta_{2} \alpha H_{f}^{-\frac{1}{2}} A^{-} \Phi_{*}^{2} \cdot \nabla u_{\alpha}, H_{f}^{\frac{1}{2}} \pi^{1} \Delta_{\sharp}^{\perp}\right\rangle\right| \leq \epsilon|| H_{f}^{\frac{1}{2}} \pi^{1} \Delta_{\sharp}^{\perp} \|^{2}+c \alpha^{5}\left|\eta_{2}\right|^{2} .
\end{aligned}
$$

Collecting (61) to (67) concludes the proof.

\subsection{Estimate for the Term $\left\langle H \Psi^{\perp}, \Psi^{\perp}\right\rangle$}

\section{Lemma 7.4.}

$$
\left\langle H \Psi^{\perp}, \Psi^{\perp}\right\rangle \geq\left(\Sigma_{0}-e_{0}\right)\left\|\Delta_{\sharp}^{\perp}\right\|^{2}-c \alpha^{4}\left|\kappa_{1}\right|^{2}+\frac{1}{2} M\left[\Delta_{\sharp}^{\perp}\right]+\left|\kappa_{1}\right|^{2}\left\|\Phi_{\sharp}^{u_{\alpha}}\right\|_{\sharp}^{2},
$$

where $M[$.$] is defined in Corollary 4.2.$

Proof. Recall that

$$
\begin{aligned}
H= & \left(P^{2}-\frac{\alpha}{|x|}\right)+\left(H_{f}+P_{f}^{2}\right)-2 \operatorname{Re}\left(P \cdot P_{f}\right) \\
& -2 \sqrt{\alpha}\left(P-P_{f}\right) \cdot A(0)+2 \alpha A^{+} \cdot A^{-}+2 \alpha \operatorname{Re}\left(A^{-}\right)^{2} .
\end{aligned}
$$


Due to the orthogonality $\left\langle\Phi_{\sharp}^{u_{\alpha}}, \Delta_{\sharp}^{\perp}\right\rangle_{\sharp}=0$, we get

$$
\begin{aligned}
\left\langle H \Psi^{\perp}, \Psi^{\perp}\right\rangle & \\
= & \langle H r, r\rangle+\left|\kappa_{1}\right|^{2}\left\|\Phi_{\sharp}^{u_{\alpha}}\right\|_{\sharp}^{2}-e_{0}\left|\kappa_{1}\right|^{2}\left\|\Phi_{\sharp}^{u_{\alpha}}\right\|^{2}-e_{0} \kappa_{1}\left\langle\Delta_{\sharp}^{\perp}, \Phi_{\sharp}^{u_{\alpha}}\right\rangle \\
& +2 \alpha\left|\kappa_{1}\right|^{2}\left\|A^{-} \Phi_{\sharp}^{u_{\alpha}}\right\|^{2} \\
& -2 \operatorname{Re}\left\langle P \cdot P_{f} \Phi_{\sharp}^{u_{\alpha}}, \Phi_{\sharp}^{u_{\alpha}}\right\rangle+2 \alpha \operatorname{Re}\left\langle A^{-} \cdot A^{-} \Delta_{\sharp}^{\perp}, \kappa_{1} \Phi_{\sharp}^{u_{\alpha}}\right\rangle \\
& -4 \sqrt{\alpha} \operatorname{Re}\left\langle P \cdot A^{-} \Delta_{\sharp}^{\perp}, \kappa_{1} \Phi_{\sharp}^{u_{\alpha}}\right\rangle \\
& -4 \sqrt{\alpha} \operatorname{Re}\left\langle P \cdot A^{+} \Delta_{\sharp}^{\perp}, \kappa_{1} \Phi_{\sharp}^{u_{\alpha}}\right\rangle+4 \operatorname{Re} \sqrt{\alpha}\left\langle P_{f} \cdot A(0) \Delta_{\sharp}^{\perp}, \kappa_{1} \Phi_{\sharp}^{u_{\alpha}}\right\rangle \\
& +4 \alpha \operatorname{Re}\left\langle A^{+} \cdot A^{-} \Delta_{\sharp}^{\perp}, \kappa_{1} \Phi_{\sharp}^{u_{\alpha}}\right\rangle-4 \operatorname{Re}\left\langle P \cdot P_{f} \Phi_{\sharp}^{u_{\alpha}}, \Delta_{\sharp}^{\perp}\right\rangle .
\end{aligned}
$$

For the first term on the right-hand side of (69), we have, from Corollary 4.2

$$
\left\langle H \Delta_{\sharp}^{\perp}, \Delta_{\sharp}^{\perp}\right\rangle \geq\left(\Sigma_{0}-e_{0}\right)\left\|\Delta_{\sharp}^{\perp}\right\|^{2}+M\left[\Delta_{\sharp}^{\perp}\right] .
$$

According to Lemma C.4, we obtain

$$
-e_{0} \kappa_{1}\left\langle\Delta_{\sharp}^{\perp}, \Phi_{\sharp}^{u_{\alpha}}\right\rangle-e_{0}\left|\kappa_{1}\right|^{2}\left\|\Phi_{\sharp}^{u_{\alpha}}\right\|^{2} \geq-\epsilon \alpha^{2}\left\|\Delta_{\sharp}^{\perp}\right\|^{2}-c\left|\kappa_{1}\right|^{2} \alpha^{5} \log \alpha^{-1} .
$$

The next term, namely $2 \alpha\left|\kappa_{1}\right|^{2}\left\|A^{-} \Phi_{\sharp}^{u_{\alpha}}\right\|^{2}$, is positive.

Due to the symmetry in $x$-variable,

$$
\left\langle P . P_{f} \Phi_{\sharp}^{u_{\alpha}}, \Phi_{\sharp}^{u_{\alpha}}\right\rangle=0 .
$$

The term $2 \alpha \operatorname{Re}\left\langle A^{-} \cdot A^{-} \Delta_{\sharp}^{\perp}, \kappa_{1} \Phi_{\sharp}^{u_{\alpha}}\right\rangle$ is estimated as

$$
\begin{aligned}
& 2 \alpha \operatorname{Re}\left\langle A^{-} \cdot A^{-} \Delta_{\sharp}^{\perp}, \kappa_{1} \Phi_{\sharp}^{u_{\alpha}}\right\rangle \\
& \quad \geq-c \alpha^{2}\left\|\kappa_{1} \Phi_{\sharp}^{u_{\alpha}}\right\|^{2}-\frac{1}{4} \nu\left\|H_{f}^{\frac{1}{2}} \Delta_{\sharp}^{\perp}\right\|^{2}=-\frac{1}{4} \nu\left\|H_{f}^{\frac{1}{2}} \Delta_{\sharp}^{\perp}\right\|^{2}-c\left|\kappa_{1}\right|^{2} \alpha^{5} \log \alpha^{-1} .
\end{aligned}
$$

Due to Lemma C.4 and [13, Lemma A4],

$$
\left|\alpha^{\frac{1}{2}}\left\langle A^{-} \Delta_{\sharp}^{\perp}, P \kappa_{1} \Phi_{\sharp}^{u_{\alpha}}\right\rangle\right| \leq \frac{\nu}{8}\left\|H_{f}^{\frac{1}{2}} \Delta_{\sharp}^{\perp}\right\|^{2}+c\left|\kappa_{1}\right|^{2} \alpha^{6} \log \alpha^{-1} .
$$

The next term we have to estimate fulfils

$$
\begin{aligned}
& \left|\alpha^{\frac{1}{2}}\left\langle P . A^{+} \Delta_{\sharp}^{\perp}, \kappa_{1} \Phi_{\sharp}^{u_{\alpha}}\right\rangle\right| \\
& \quad \leq \epsilon\left\|P \pi^{0} \Delta_{\sharp}^{\perp}\right\|^{2}+c \alpha\left|\kappa_{1}\right|^{2}\left\|A^{-} \Phi_{\sharp}^{u_{\alpha}}\right\|^{2} \leq \epsilon\left\|\left(P-P_{f}\right) \Delta_{\sharp}^{\perp}\right\|^{2}-c\left|\kappa_{1}\right|^{2} \alpha^{4} .
\end{aligned}
$$

We have

$$
\begin{aligned}
\operatorname{Re} & \sqrt{\alpha}\left\langle P_{f} \cdot A(0) \Delta_{\sharp}^{\perp}, \kappa_{1} \Phi_{\sharp}^{u_{\alpha}}\right\rangle \\
& =\operatorname{Re} \sqrt{\alpha}\left\langle P_{f} \cdot A^{-} \pi^{2} \Delta_{\sharp}^{\perp}, \kappa_{1} \Phi_{\sharp}^{u_{\alpha}}\right\rangle+\operatorname{Re} \sqrt{\alpha}\left\langle P_{f} \cdot A^{+} \pi^{0} \Delta_{\sharp}^{\perp}, \kappa_{1} \Phi_{\sharp}^{u_{\alpha}}\right\rangle \\
& =\operatorname{Re} \sqrt{\alpha}\left\langle P_{f} \cdot A^{-} \pi^{2} \Delta_{\sharp}^{\perp}, \kappa_{1} \Phi_{\sharp}^{u_{\alpha}}\right\rangle+\operatorname{Re} \sqrt{\alpha}\left\langle A^{+} \cdot P_{f} \pi^{0} \Delta_{\sharp}^{\perp}, \kappa_{1} \Phi_{\sharp}^{u_{\alpha}}\right\rangle
\end{aligned}
$$

Obviously, $\left\langle A^{+} . P_{f} \pi^{0} \Delta_{\sharp}^{\perp}, \kappa_{1} \Phi_{\sharp}^{u_{\alpha}}\right\rangle=0$, and the first term is bounded by

$$
\begin{aligned}
\left|\operatorname{Re} \sqrt{\alpha}\left\langle P_{f} \cdot A^{-} \pi^{2} \Delta_{\sharp}^{\perp}, \kappa_{1} \Phi_{\sharp}^{u_{\alpha}}\right\rangle\right| & \leq \epsilon\left\|H_{f}^{\frac{1}{2}} \pi^{2} \Delta_{\sharp}^{\perp}\right\|^{2}+c \alpha\left|\kappa_{1}\right|^{2}\left\|P_{f} \Phi_{\sharp}^{u_{\alpha}}\right\|^{2} \\
& \leq \epsilon\left\|H_{f}^{\frac{1}{2}} \pi^{2} \Delta_{\sharp}^{\perp}\right\|^{2}+c\left|\kappa_{1}\right|^{2} \alpha^{4} .
\end{aligned}
$$


For the term $\alpha \operatorname{Re}\left\langle A^{+} . A^{-} \Delta_{\sharp}^{\perp}, \kappa_{1} \Phi_{\sharp}^{u_{\alpha}}\right\rangle$ we obtain

$$
\begin{aligned}
& \alpha \operatorname{Re}\left\langle A^{+} . A^{-} \Delta_{\sharp}^{\perp}, \kappa_{1} \Phi_{\sharp}^{u_{\alpha}}\right\rangle \\
& \quad \geq-c \alpha^{2}\left\|H_{f}^{\frac{1}{2}} \kappa_{1} \Phi_{\sharp}^{u_{\alpha}}\right\|^{2}\left|\kappa_{1}\right|^{2}-\epsilon\left\|H_{f}^{\frac{1}{2}} \Delta_{\sharp}^{\perp}\right\|^{2}=-c \alpha^{5}\left|\kappa_{1}\right|^{2}-\epsilon\left\|H_{f}^{\frac{1}{2}} \Delta_{\sharp}^{\perp}\right\|^{2} .
\end{aligned}
$$

According to (213) of Lemma C.4, the last term we have to estimate fulfils

$$
\begin{aligned}
& \operatorname{Re}\left\langle P . P_{f} \kappa_{1} \Phi_{\sharp}^{u_{\alpha}}, \Delta_{\sharp}^{\perp}\right\rangle \\
& \quad \leq \epsilon\left\|H_{f}^{\frac{1}{2}} \pi^{1} \Delta_{\sharp}^{\perp}\right\|^{2}+c\left\|P\left|P_{f}\right|^{\frac{1}{2}} \kappa_{1} \Phi_{\sharp}^{u_{\alpha}}\right\|^{2} \leq \epsilon\left\|H_{f}^{\frac{1}{2}} \pi^{1} \Delta_{\sharp}^{\perp}\right\|^{2}+c \alpha^{5}\left|\kappa_{1}\right|^{2} .
\end{aligned}
$$

Collecting the estimates (69) to (79) yields (68).

\subsection{Upper Bound on the Binding Energy with Error Term $\mathcal{O}\left(\alpha^{4}\right)$}

We first establish a lemma that we shall need in the proof of Theorem 5.2 in order to improve the error term from $\mathcal{O}\left(\alpha^{4} \log \alpha^{-1}\right)$ to $\mathcal{O}\left(\alpha^{4}\right)$.

Lemma 7.5. If $\left\|\Delta_{*}^{u_{\alpha}}\right\|_{*}^{2}=\mathcal{O}\left(\alpha^{4} \log \alpha^{-1}\right)$ and $\left\|H_{f}^{\frac{1}{2}} \Delta_{\sharp}^{\perp}\right\|^{2}=\mathcal{O}\left(\alpha^{4} \log \alpha^{-1}\right)$ hold, then we have

$$
\begin{aligned}
\left\|N_{f}^{\frac{1}{2}} \Delta_{*}^{u_{\alpha}}\right\|^{2} & =\mathcal{O}\left(\alpha^{\frac{33}{16}}\right) \\
\left\|N_{f}^{\frac{1}{2}} \Delta_{\sharp}^{\perp}\right\|^{2} & =\mathcal{O}\left(\alpha^{\frac{33}{16}}\right)
\end{aligned}
$$

Proof. We note that from Definition 5.1, we have

$$
\left\|a_{\lambda}(k) \Psi^{u_{\alpha}}\right\|^{2} \leq\left\|a_{\lambda}(k) \Psi^{\mathrm{GS}}\right\|^{2} \leq \frac{c \alpha^{-\frac{3}{2}} \chi_{\Lambda}(|k|)}{|k|},
$$

where in the last inequality, we used (14). Taking into account that

$$
\Delta_{*}^{u_{\alpha}}=\Psi^{u_{\alpha}}-2 \eta_{1} \alpha^{\frac{3}{2}} \Phi_{*}^{1}-\eta_{2} \alpha \Phi_{*}^{2}-2 \eta_{3} \alpha^{\frac{3}{2}} \Phi_{*}^{3},
$$

where

$$
\begin{gathered}
\left\|a_{\lambda}(k) \Phi_{*}^{1}\right\|^{2} \leq \frac{c \chi_{\Lambda}(|k|)}{|k|},\left\|\quad a_{\lambda}(k) \Phi_{*}^{2}\right\|^{2} \leq \frac{c \chi_{\Lambda}(|k|)(1+|\log | k||)}{|k|} \\
\left\|a_{\lambda}(k) \Phi_{*}^{3}\right\|^{2} \leq \frac{c \chi_{\Lambda}(|k|)}{|k|}
\end{gathered}
$$

and using (85), we arrive at

$$
\left\|a_{\lambda}(k) \Delta_{*}^{u_{\alpha}}\right\|^{2} \leq \frac{c \alpha^{-\frac{3}{2}} \chi_{\Lambda}(|k|)(1+|\log | k||)}{|k|} .
$$

For the expected photon number of $\Delta_{*}^{u_{\alpha}}$ thus holds

$$
\begin{aligned}
& \left\|N_{f}^{\frac{1}{2}} \Delta_{*}^{u_{\alpha}}\right\|^{2} \\
& =\sum_{\lambda} \int\left\|a_{\lambda}(k) \Delta_{*}^{u_{\alpha}}\right\|^{2} \mathrm{~d} k
\end{aligned}
$$




$$
\begin{aligned}
& \leq \sum_{\lambda} \int_{|k| \leq \alpha^{\frac{15}{8}}} \frac{c \alpha^{-\frac{3}{2}}(1+|\log | k||)}{|k|} \mathrm{d} k+\int_{|k|>\alpha^{\frac{15}{8}}}|k|^{-1}|k|\left\|a_{\lambda}(k) \Delta_{*}^{u_{\alpha}}\right\|^{2} \mathrm{~d} k \\
& \leq c \alpha^{\frac{17}{8}}+c \alpha^{-\frac{15}{8}}\left\|H_{f}^{\frac{1}{2}} \Delta_{*}^{u_{\alpha}}\right\|^{2} \leq c \alpha^{\frac{33}{16}},
\end{aligned}
$$

using (87) in the last inequality. The relation (81) can be proved similarly, using

$$
\left\|a_{\lambda}(k) \Phi_{\sharp}^{u_{\alpha}}\right\|^{2} \leq c \frac{\alpha^{-1}}{|k|} .
$$

This concludes the proof of the lemma.

\subsection{Concluding the Proof of Theorem 5.2}

The proof of Theorem 5.2 is obtained in the following two steps: We first show that the estimate holds with an error term $\mathcal{O}\left(\alpha^{4} \log \alpha^{-1}\right)$. In a second step, using Lemma 7.5, we improve to an error term $\mathcal{O}\left(\alpha^{4}\right)$. Then, we derive the estimates (45)-(48) that shall be used in the next section for the computation of the binding energy to higher order.

- Step 1: We first show that (44) holds with an error estimate of the order $\alpha^{4} \log \alpha^{-1}$.

Collecting Lemmata 7.1, 7.2, 7.3 and Lemma 7.4 yields

$$
\begin{aligned}
& \left\langle H \Psi^{\mathrm{GS}}, \Psi^{\mathrm{GS}}\right\rangle \\
& \geq-e_{0}\left\|\Psi^{u_{\alpha}}\right\|^{2}-\alpha^{2}\left\|\Phi_{*}^{2}\right\|_{*}^{2}\left|\pi^{0} \Psi^{u_{\alpha}}\right|^{2}+\alpha^{3}\left|\eta_{2}\right|^{2}\left(2\left\|A^{-} \Phi_{*}^{2}\right\|^{2}-4\left\|\Phi_{*}^{1}\right\|_{*}^{2}-4\left\|\Phi_{*}^{3}\right\|_{*}^{2}\right) \\
& \quad+\alpha^{2}\left|\eta_{2}-\pi^{0} \Psi^{u_{\alpha}}\right|^{2}\left\|\Phi_{*}^{2}\right\|_{*}^{2}+4 \alpha^{3}\left(\left|\eta_{1}-\eta_{2}\right|^{2}\left\|\Phi_{*}^{1}\right\|_{*}^{2}+\left|\eta_{3}-\eta_{2}\right|^{2}\left\|\Phi_{*}^{3}\right\|_{*}^{2}\right) \\
& \quad-c \alpha^{4} \log \alpha^{-1}\left(\left|\eta_{1}\right|^{2}+\left|\eta_{2}\right|^{2}+\left|\eta_{3}\right|^{2}+\left|\pi^{0} \Psi^{u_{\alpha}}\right|^{2}\right)+\frac{1}{4}\left\|\Delta_{*}^{u_{\alpha}}\right\|_{*}^{2} \\
& \quad+\left|\kappa_{1}\right|^{2}\left\|\Phi_{\sharp}^{u_{\alpha}}\right\|_{\sharp}^{2}+\left(\Sigma_{0}-e_{0}\right)\left\|\Delta_{\sharp}^{\perp}\right\|^{2}+\frac{1}{4} M\left[\Delta_{\sharp}^{\perp}\right]-c \alpha^{4}\left|\kappa_{1}\right|^{2} \\
& \quad-2 \operatorname{Re}\left(\kappa_{1} \pi^{0} \Psi^{u_{\alpha}}\right)\left\|\Phi_{\sharp}^{u_{\alpha}}\right\|_{\sharp}^{2}+\mathcal{O}\left(\alpha^{5} \log \alpha^{-1}\right) .
\end{aligned}
$$

We first estimate

$$
\begin{aligned}
& \left|\kappa_{1}\right|^{2}\left\|\Phi_{\sharp}^{u_{\alpha}}\right\|_{\sharp}^{2}-2 \operatorname{Re}\left(\overline{\kappa_{1}} \pi^{0} \Psi^{u_{\alpha}}\right)\left\|\Phi_{\sharp}^{u_{\alpha}}\right\|_{\sharp}^{2}-c \alpha^{4}\left|\kappa_{1}\right|^{2} \\
& \geq-\left\|\Phi_{\sharp}^{u_{\alpha}}\right\|_{\sharp}^{2}\left|\pi^{0} \Psi^{u_{\alpha}}\right|^{2}+\frac{\left|\overline{\kappa_{1}}-\pi^{0} \Psi^{u_{\alpha}}\right|^{2}}{2}\left\|\Phi_{\sharp}^{u_{\alpha}}\right\|_{\sharp}^{2}+\mathcal{O}\left(\alpha^{4}\right) .
\end{aligned}
$$

Moreover, since $\left|\pi^{0} \Psi^{u_{\alpha}}\right| \leq 1$, we replace in (82) $-\alpha^{2}\left\|\Phi_{*}^{2}\right\|_{*}^{2}\left|\pi^{0} \Psi^{u_{\alpha}}\right|^{2}$ by $-\alpha^{2}\left\|\Phi_{*}^{2}\right\|_{*}^{2}$ and in (83) $-\left\|\Phi_{\sharp}^{u_{\alpha}}\right\|_{\sharp}^{2}\left|\pi^{0} \Psi^{u_{\alpha}}\right|^{2}$ by $-\left\|\Phi_{\sharp}^{u_{\alpha}}\right\|_{\sharp}^{2}$. In addition, using the inequalities

$\left|\eta_{2}-\eta_{j}\right|^{2} \geq \frac{1}{2}\left|\eta_{j}-\pi^{0} \Psi^{u_{\alpha}}\right|^{2}-\left|\eta_{2}-\pi^{0} \Psi^{u_{\alpha}}\right|^{2} \quad$ and $\quad\left|\eta_{j}\right|^{2} \leq 2\left|\eta_{j}-\pi^{0} \Psi^{u_{\alpha}}\right|^{2}+2$

for $j=1,2,3$ yields that for some $c>0$,

$$
\begin{aligned}
& \left\langle H \Psi^{\mathrm{GS}}, \Psi^{\mathrm{GS}}\right\rangle \\
& \geq-e_{0}\left\|\Psi^{u_{\alpha}}\right\|^{2}-\alpha^{2}\left\|\Phi_{*}^{2}\right\|_{*}^{2}+\alpha^{3}\left|\eta_{2}\right|^{2}\left(2\left\|A^{-} \Phi_{*}^{2}\right\|^{2}\right. \\
& \left.-4\left\|\Phi_{*}^{1}\right\|_{*}^{2}-4\left\|\Phi_{*}^{3}\right\|_{*}^{2}\right)-\left\|\Phi_{\sharp}^{u_{\alpha}}\right\|_{\sharp}^{2}
\end{aligned}
$$




$$
\begin{aligned}
& +c \alpha^{2}\left|\eta_{2}-\pi^{0} \Psi^{u_{\alpha}}\right|^{2}\left\|\Phi_{*}^{2}\right\|_{*}^{2}+c \alpha^{3}\left(\left|\eta_{1}-\pi^{0} \Psi^{u_{\alpha}}\right|^{2}+\left|\eta_{3}-\pi^{0} \Psi^{u_{\alpha}}\right|^{2}\right) \\
& +c \alpha^{3}\left|\kappa_{1}-\pi^{0} \Psi^{u_{\alpha}}\right|^{2}+\frac{1}{4}\left\|\Delta_{*}^{u_{\alpha}}\right\|_{*}^{2}+\left(\Sigma_{0}-e_{0}\right)\left\|\Delta_{\sharp}^{\perp}\right\|^{2} \\
& +\frac{1}{4} M\left[\Delta_{\sharp}^{\perp}\right]+\mathcal{O}\left(\alpha^{4} \log \alpha^{-1}\right) .
\end{aligned}
$$

Comparing this expression with (43) of Theorem 5.1 gives

$$
\max \left\{\left|\eta_{1}\right|,\left|\eta_{2}\right|, \mid \eta_{3}\right\} \leq 2
$$

and

$$
\Sigma=\Sigma_{0}-e_{0}-\left\|\Phi_{\sharp}^{u_{\alpha}}\right\|_{\sharp}^{2}+\mathcal{O}\left(\alpha^{4} \log \alpha^{-1}\right) .
$$

Finally, by Lemma C.5, we can replace $\left\|\Phi_{\sharp}^{u_{\alpha}}\right\|_{\sharp}$ by $\left\|\Phi_{*}^{u_{\alpha}}\right\|_{*}$ in the above equality, which proves (44) with an error term $\mathcal{O}\left(\alpha^{4} \log \alpha^{-1}\right)$.

- Step 2: We now show that the error term does not contain any $\log \alpha^{-1}$ term.

From (84) we obtain

$$
\left\|\Delta_{*}^{u_{\alpha}}\right\|_{*}^{2}=\mathcal{O}\left(\alpha^{4} \log \alpha^{-1}\right) \quad \text { and } \quad\left\|H_{f}^{\frac{1}{2}} \Delta_{\sharp}^{\perp}\right\|^{2}=\mathcal{O}\left(\alpha^{4} \log \alpha^{-1}\right) .
$$

According to Lemma 7.5, this implies

$$
\left\|N_{f}^{\frac{1}{2}} \Delta_{*}^{u_{\alpha}}\right\|^{2}=\mathcal{O}\left(\alpha^{\frac{33}{16}}\right) \text { and }\left\|N_{f}^{\frac{1}{2}} \Delta_{\sharp}^{\perp}\right\|^{2}=\mathcal{O}\left(\alpha^{\frac{33}{16}}\right) .
$$

Thus, we have

$$
\begin{aligned}
& \left\|N_{f}^{\frac{1}{2}} \Psi^{u_{\alpha}}\right\|^{2} \\
& \quad \leq 4\left|\eta_{1}\right|^{2} \alpha^{3}\left\|N^{\frac{1}{2}} \Phi_{*}^{1}\right\|^{2}+\left|\eta_{2}\right|^{2} \alpha^{2}\left\|N^{\frac{1}{2}} \Phi_{*}^{2}\right\|^{2}+4\left|\eta_{3}\right|^{2} \alpha^{3}\left\|N^{\frac{1}{2}} \Phi_{*}^{3}\right\|^{2}+\left\|N^{\frac{1}{2}} \Delta_{*}^{u_{\alpha}}\right\|^{2} \\
& \quad=\mathcal{O}\left(\alpha^{2}\right),
\end{aligned}
$$

which implies that in Lemma 7.1 we can replace the term $c \alpha^{4} \log \alpha^{-1}\left(\left|\eta_{1}\right|^{2}+\right.$ $\left.\left|\eta_{2}\right|^{2}+\left|\eta_{3}\right|^{2}+\left|\pi^{0} \Psi^{u_{\alpha}}\right|^{2}\right)$ with $c \alpha^{4}\left(\left|\eta_{1}\right|^{2}+\left|\eta_{2}\right|^{2}+\left|\eta_{3}\right|^{2}+\left|\pi^{0} \Psi^{u_{\alpha}}\right|^{2}\right)$ and consequently in (84) and (86), the term $\mathcal{O}\left(\alpha^{4} \log \alpha^{-1}\right)$ can be replaced by $\mathcal{O}\left(\alpha^{4}\right)$. This proves (44). The estimates (45)-(48) follow from (44) and (84) with $\mathcal{O}\left(\alpha^{4} \log \alpha^{-1}\right)$ replaced with $\mathcal{O}\left(\alpha^{4}\right)$. We thus arrive at the proof of Theorem 5.2.

\section{Proof of Theorem 6.1}

In this section, we prove the upper bound on the binding energy up to the order $\alpha^{5} \log \alpha^{-1}$ provided in Theorem 6.1.

We have

$$
\left\langle H \Psi^{\mathrm{GS}}, \Psi^{\mathrm{GS}}\right\rangle \geq\left(\Sigma_{0}-e_{0}\right)\left\|\Psi^{u_{\alpha}}\right\|^{2}+2 \operatorname{Re}\left\langle H \Psi^{\perp}, u_{\alpha} \Psi^{u_{\alpha}}\right\rangle+\left\langle H \Psi^{\perp}, \Psi^{\perp}\right\rangle .
$$

The estimates for the last two terms in (88) are given in Propositions 6.1 and 6.2. We will bound below this expression by considering separately the terms involving the parameters $\kappa_{1}, \kappa_{2, i}$, and $\kappa_{3}$. 
- We first estimate the second term on the right-hand side of (54) together with the seventh term on the right-hand side of (55). We have

$$
\begin{aligned}
& -4 \alpha \operatorname{Re}\left\langle\nabla u_{\alpha} \cdot P_{f} \Phi_{*}^{2}, \pi^{2} \Psi_{2}^{\perp}\right\rangle+4 \alpha^{\frac{1}{2}} \operatorname{Re}\left\langle\pi^{2} \Psi_{2}^{\perp}, A^{+} . P_{f} \Phi_{*}^{u_{\alpha}}\right\rangle \\
& =-4 \alpha \operatorname{Re}\left\langle\pi^{2} \Psi_{2}^{\perp},\left(\sum_{i=1}^{3} P_{f}^{i} \Phi_{*}^{2}-2 A^{+} . P_{f}\left(H_{f}+P_{f}^{2}\right)^{-1}\left(A^{+}\right)^{i} \Omega_{f}\right) \frac{\partial u_{\alpha}}{\partial x_{i}}\right\rangle \\
& =-4 \alpha \sum_{i=1}^{3} \operatorname{Re}\left\langle\pi^{2} \Psi_{2}^{\perp},\left(H_{f}+P_{f}^{2}\right)^{-1} W_{i} \frac{\partial u_{\alpha}}{\partial x_{i}}\right\rangle_{*} .
\end{aligned}
$$

Using the $\langle\cdot, \cdot\rangle_{\sharp}$-orthogonality of $\pi^{2} \Psi_{2}^{\perp}$ and $\left(H_{f}+P_{f}^{2}\right)^{-1} W_{i} \frac{\partial u_{\alpha}}{\partial x_{i}}$, the last expression can be estimated as

$$
-4 \alpha \sum_{i=1}^{3} \operatorname{Re}\left\langle\pi^{2} \Psi_{2}^{\perp},\left(h_{\alpha}+e_{0}\right)\left(H_{f}+P_{f}^{2}\right)^{-1} W_{i} \frac{\partial u_{\alpha}}{\partial x_{i}}\right\rangle .
$$

By the Schwarz inequality, this term is bounded below by

$$
-\epsilon \alpha^{2}\left\|\pi^{2} \Psi_{2}^{\perp}\right\|^{2}-c \sum_{i=1}^{3}\left\|\left(h_{\alpha}+e_{0}\right) \frac{\partial u_{\alpha}}{\partial x_{i}}\right\|^{2}=-\epsilon \alpha^{2}\left\|\pi^{2} \Psi_{2}^{\perp}\right\|^{2}-\mathcal{O}\left(\alpha^{6}\right) .
$$

- Next, we collect all the terms involving $\kappa_{1}$ in (54) and (55). This yields

$$
\begin{aligned}
& -2 \operatorname{Re} \overline{\kappa_{1}} \pi^{0} \Psi^{u_{\alpha}}\left\|\Phi_{\sharp}^{u_{\alpha}}\right\|_{\sharp}^{2}+\left|\kappa_{1}\right|^{2}\left\|\Phi_{\sharp}^{u_{\alpha}}\right\|_{\sharp}^{2}-\left|\kappa_{1}-1\right| c \alpha^{4} \\
& \quad \geq-\left|\pi^{0} \Psi^{u_{\alpha}}\right|^{2}\left\|\Phi_{\sharp}^{u_{\alpha}}\right\|_{\sharp}^{2}+\left|\kappa_{1}-\pi^{0} \Psi^{u_{\alpha}}\right|^{2}\left\|\Phi_{\sharp}^{u_{\alpha}}\right\|_{\sharp}^{2}-\left|\kappa_{1}-1\right| c \alpha^{4}
\end{aligned}
$$

Notice that from Theorem 5.2 we have $\left|\pi^{0} \Psi^{u_{\alpha}}\right|^{2}=1+\mathcal{O}\left(\alpha^{2}\right)$; moreover, we have $\left|\pi^{0} \Psi^{u_{\alpha}}\right|=1+\mathcal{O}\left(\alpha^{2}\right)$. This yields

$$
\begin{aligned}
& -2 \operatorname{Re} \overline{\kappa_{1}} \pi^{0} \Psi^{u_{\alpha}}\left\|\Phi_{\sharp}^{u_{\alpha}}\right\|_{\sharp}^{2}+\left|\kappa_{1}\right|^{2}\left\|\Phi_{\sharp}^{u_{\alpha}}\right\|_{\sharp}^{2}-\left|\kappa_{1}-1\right| c \alpha^{4} \\
& \quad \geq-\left\|\Phi_{\sharp}^{u_{\alpha}}\right\|_{\sharp}^{2}+\left|\kappa_{1}-1\right|^{2} c^{\prime} \alpha^{3}-\left|\kappa_{1}-1\right| c \alpha^{4}+\mathcal{O}\left(\alpha^{5}\right) \geq-\left\|\Phi_{\sharp}^{u_{\alpha}}\right\|_{\sharp}^{2}+\mathcal{O}\left(\alpha^{5}\right) .
\end{aligned}
$$

- We now collect and estimate the terms in (54) and (55) involving $\kappa_{2, i}$. We get

$$
\begin{aligned}
& -\frac{1}{3} \alpha^{4} \operatorname{Re} \sum_{i=1}^{3} \kappa_{2, i}\left\langle\left(H_{f}+P_{f}^{2}\right)^{-1} W_{i}, P_{f}^{i} \Phi_{*}^{2}\right\rangle+\frac{\alpha^{4}}{12} \sum_{i=1}^{3}\left|\kappa_{2, i}\right|^{2}\left\|\left(H_{f}+P_{f}^{2}\right)^{-1} W_{i}\right\|_{*}^{2} \\
& \quad+\frac{2}{3} \alpha^{4} \operatorname{Re} \sum_{i=1}^{3} \kappa_{2, i}\left\langle\left(H_{f}+P_{f}^{2}\right)^{-1} W_{i}, P_{f} \cdot A^{+}\left(H_{f}+P_{f}^{2}\right)^{-1}\left(A^{+}\right)^{i} \Omega_{f}\right\rangle \\
& =-\frac{1}{3} \alpha^{4} \operatorname{Re} \sum_{i=1}^{3} \kappa_{2, i}\left\|\left(H_{f}+P_{f}^{2}\right)^{-1} W_{i}\right\|_{*}^{2}+\frac{\alpha^{4}}{12} \sum_{i=1}^{3}\left|\kappa_{2, i}\right|^{2}\left\|\left(H_{f}+P_{f}^{2}\right)^{-1} W_{i}\right\|_{*}^{2} \\
& \geq-\frac{\alpha^{4}}{3} \sum_{i=1}^{3}\left\|\left(H_{f}+P_{f}^{2}\right)^{-1} W_{i}\right\|_{*}^{2}
\end{aligned}
$$


- Collecting in (54) and (55) the terms containing $\kappa_{3}$ yields

$$
\begin{aligned}
& \frac{\left|\kappa_{3}+1\right|^{2}}{2} \alpha^{2}\left\|\Phi_{*}^{2}\right\|_{*}^{2}\left\|\Phi_{\sharp}^{u_{\alpha}}\right\|^{2}-\epsilon \alpha^{5}\left|\kappa_{3}\right|^{2} \\
& \quad \geq c_{1} \alpha^{5} \log \alpha^{-1}\left|\kappa_{3}+1\right|^{2}-\epsilon \alpha^{5}\left|\kappa_{3}\right|^{2} \geq-c_{2} \alpha^{5},
\end{aligned}
$$

where $c_{1}$ and $c_{2}$ are positive constants.

- The fifth term on the right-hand side of (54) and the first term on the right-hand side of (56) are estimated, for $\alpha$ small enough, as

$$
\left(1-c_{0} \alpha\right)\left\|\left(h_{\alpha}+e_{0}\right)^{\frac{1}{2}} \pi^{0}\left(\Psi_{1}^{\perp}\right)^{a}\right\|^{2}-\epsilon \alpha^{2}\left\|\left(\Psi_{1}^{\perp}\right)^{a}\right\|^{2} \geq\left(\frac{\delta}{2}-\epsilon \alpha^{2}\right)\left\|\left(\Psi_{1}^{\perp}\right)^{a}\right\|^{2} \geq 0,
$$

with $\delta=\frac{3}{32} \alpha^{2}$, and where we used that $\left(\Psi_{1}^{\perp}\right)^{a}$ is orthogonal to $u_{\alpha}$.

- Substituting the above estimates in (88) yields

$$
\begin{aligned}
& \left\langle H \Psi^{\mathrm{GS}}, \Psi^{\mathrm{GS}}\right\rangle \\
& \geq\left(\Sigma_{0}-e_{0}\right)\left\|\Psi^{u_{\alpha}}\right\|^{2}+\left(\Sigma_{0}-e_{0}\right)\left\|\Psi^{\perp}\right\|^{2}-\left\|\Phi_{\sharp}^{u_{\alpha}}\right\|_{\sharp}^{2} \\
& \quad-\alpha^{4} \sum_{i=1}^{3}\left\|\left(H_{f}+P_{f}^{2}\right)^{-1} W_{i}\right\|_{*}^{2} \\
& \quad-\frac{2}{3} \alpha^{4} \operatorname{Re} \sum_{i=1}^{3}\left\langle\left(H_{f}+P_{f}^{2}\right)^{-\frac{1}{2}}\left(A^{-}\right)^{i} \Phi_{*}^{2},\left(H_{f}+P_{f}^{2}\right)^{-\frac{1}{2}}\left(A^{+}\right)^{i} \Omega_{f}\right\rangle \\
& \quad-4 \alpha\left\|\left(h_{\alpha}+e_{0}\right)^{-\frac{1}{2}} Q_{\alpha}^{\perp} P A^{-} \Phi_{*}^{u_{\alpha}}\right\|^{2}+2 \alpha\left\|A^{-} \Phi_{*}^{u_{\alpha}}\right\|^{2}+o\left(\alpha^{5} \log \alpha^{-1}\right),
\end{aligned}
$$

where $Q_{\alpha}^{\perp}$ is the projection onto the orthogonal complement to the ground state $u_{\alpha}$ of the Schrödinger operator $h_{\alpha}=-\Delta-\frac{\alpha}{|x|}$.

To complete the proof of Theorem 6.1 we first note that

$$
\left\|\Psi^{u_{\alpha}}\right\|^{2}+\left\|\Psi^{\perp}\right\|^{2}=\left\|\Psi^{\mathrm{GS}}\right\|^{2} .
$$

Moreover, according to Lemma C.5

$$
-\left\|\Phi_{\sharp}^{u_{\alpha}}\right\|_{\sharp}^{2}=-\left\|\Phi_{*}^{u_{\alpha}}\right\|_{*}^{2}+\frac{1}{3 \pi}\left\|\left(h_{1}+\frac{1}{4}\right)^{\frac{1}{2}} \nabla u_{1}\right\|^{2} \alpha^{5} \log \alpha^{-1}+o\left(\alpha^{5} \log \alpha^{-1}\right),
$$

and

$$
\left\|\Phi_{*}^{u_{\alpha}}\right\|_{*}^{2}=\frac{\alpha^{3}}{2 \pi} \int_{0}^{\infty} \frac{\chi_{\Lambda}(t)}{1+t} \mathrm{~d} t=e^{(1)} \alpha^{3} .
$$

In addition, we have the following identities $(i=1,2,3)$ :

$$
\begin{aligned}
& \left\|\left(H_{f}+P_{f}^{2}\right)^{-1} W_{i}\right\|_{*}^{2}=\|\left(H_{f}+P_{f}^{2}\right)^{-\frac{1}{2}} \\
& \quad \times\left(2 A^{+} \cdot P_{f}\left(H_{f}+P_{f}^{2}\right)^{-1}\left(A^{+}\right)^{i}-P_{f}^{i}\left(H_{f}+P_{f}^{2}\right)^{-1} A^{+} \cdot A^{+}\right) \Omega_{f} \|^{2},
\end{aligned}
$$


and

$$
\begin{aligned}
& -\frac{2}{3} \alpha^{4} \operatorname{Re} \sum_{i=1}^{3}\left\langle\left(H_{f}+P_{f}^{2}\right)^{-\frac{1}{2}} A^{-} \Phi_{*}^{2},\left(H_{f}+P_{f}^{2}\right)^{-\frac{1}{2}}\left(A^{+}\right)^{i} \Omega_{f}\right\rangle \\
& \quad=-\frac{2}{3} \alpha^{4} \operatorname{Re} \sum_{i=1}^{3}\left\langle A^{-}\left(H_{f}+P_{f}^{2}\right)^{-1} A^{+} . A^{+} \Omega_{f},\left(H_{f}+P_{f}^{2}\right)^{-1}\left(A^{+}\right)^{i} \Omega_{f}\right\rangle .
\end{aligned}
$$

We also have

$$
-4 \alpha\left\|\left(h_{\alpha}+e_{0}\right)^{-\frac{1}{2}} Q_{\alpha}^{\perp} P A^{-} \Phi_{*}^{u_{\alpha}}\right\|^{2}=-4 \alpha^{4} a_{0}^{2}\left\|\left(-\Delta-\frac{1}{|x|}+\frac{1}{4}\right)^{-\frac{1}{2}} Q_{1}^{\perp} \Delta u_{1}\right\|^{2},
$$

with

$$
a_{0}=\int \frac{k_{1}^{2}+k_{2}^{2}}{4 \pi^{2}|k|^{3}} \frac{2}{|k|^{2}+|k|} \chi_{\Lambda}(|k|) \mathrm{d} k_{1} \mathrm{~d} k_{2} \mathrm{~d} k_{3},
$$

and

$$
2 \alpha\left\|A^{-} \Phi_{*}^{u_{\alpha}}\right\|^{2}=\frac{2}{3} \alpha^{4} \sum_{i=1}^{3}\left\|A^{-}\left(H_{f}+P_{f}^{2}\right)^{-1}\left(A^{+}\right)^{i} \Omega_{f}\right\|^{2} .
$$

Substituting (98)-(104) into (97) finishes the proof of Theorem 6.1 and thus the proof of the upper bound in Theorem 2.1.

\section{Proof of Theorem 2.1: Lower Bound up to $o\left(\alpha^{5} \log \alpha^{-1}\right)$ for the Binding Energy}

In this section, we prove a lower bound for $\Sigma_{0}-\Sigma$ in Theorem 2.1 which coincides with the upper bound given in (97). To this end, it suffices to compute

$$
\frac{\left\langle\left(H-\Sigma_{0}+e_{0}\right) \widetilde{\Phi}^{\text {trial }}, \widetilde{\Phi}^{\text {trial }}\right\rangle}{\left\|\widetilde{\Phi}^{\text {trial }}\right\|^{2}},
$$

with the following trial function:

$$
\widetilde{\Phi}^{\text {trial }}=u_{\alpha} \Psi_{0}+\widetilde{\Psi}_{0}^{\perp},
$$

where $\Psi_{0}$ is ground state of the operator $T(0)$ (defined in (7)), with the normalization $\pi^{0} \Psi_{0}=\Omega_{f}, u_{\alpha}$ is the normalized ground state of $h_{\alpha}=-\Delta-\frac{\alpha}{|x|}$, and $\widetilde{\Psi}_{0}^{\perp}$ is defined by

$$
\begin{aligned}
& \pi^{0} \widetilde{\Psi}_{0}^{\perp}=2 \alpha^{\frac{1}{2}}\left(h_{\alpha}+e_{0}\right)^{-1} Q_{\alpha}^{\perp} P \cdot A^{-} \Phi_{*}^{u_{\alpha}}, \pi^{1} \widetilde{\Psi}_{0}^{\perp}=\Phi_{\sharp}^{u_{\alpha}}, \\
& \pi^{2} \widetilde{\Psi}_{0}^{\perp}=\alpha \Phi_{*}^{2} \pi^{0} \widetilde{\Psi}_{0}^{\perp}+\sum_{i=1}^{3} 2 \alpha\left(H_{f}+P_{f}^{2}\right)^{-1} W_{i} \frac{\partial u_{\alpha}}{\partial x_{i}}, \\
& \pi^{3} \widetilde{\Psi}_{0}^{\perp}=-\alpha\left(H_{f}+P_{f}^{2}\right)^{-1} A^{+} \cdot A^{+} \Phi_{\sharp}^{u_{\alpha}} .
\end{aligned}
$$


Where $\Phi_{*}^{u_{\alpha}}, \Phi_{\sharp}^{u_{\alpha}}, \Phi_{*}^{2}$ and $W_{i}$ are defined as in Sects. 5 and 6. Technical Lemmata used in this proof are given in Appendix C.

We compute

$$
\left\langle H \widetilde{\Phi}^{\text {trial }}, \widetilde{\Phi}^{\text {trial }}\right\rangle=\left\langle H u_{\alpha} \Psi_{0}, u_{\alpha} \Psi_{0}\right\rangle+2 \operatorname{Re}\left\langle H u_{\alpha} \Psi_{0}, \widetilde{\Psi}_{0}^{\perp}\right\rangle+\left\langle H \widetilde{\Psi}_{0}^{\perp}, \widetilde{\Psi}_{0}^{\perp}\right\rangle,
$$

and we recall

$$
\begin{aligned}
H= & h_{\alpha}+\left(H_{f}+P_{f}^{2}\right)-2 \operatorname{Re} P \cdot P_{f}-2 \alpha^{\frac{1}{2}} P \cdot A(0)+2 \alpha^{\frac{1}{2}} P_{f} \cdot A(0) \\
& +2 \alpha A^{+} \cdot A^{-}+2 \alpha\left(A^{-}\right)^{2} .
\end{aligned}
$$

- For the first term in (106), a straightforward computation shows

$$
\left\langle H u_{\alpha} \Psi_{0}, u_{\alpha} \Psi_{0}\right\rangle=\left(\Sigma_{0}-e_{0}\right)\left\|u_{\alpha}\right\|^{2}\left\|\Psi_{0}\right\|^{2} .
$$

- We estimate the second term on the right-hand side of (106) by computing each term that occurs in the decomposition (107).

$\diamond$ Using the symmetry of $u_{\alpha}$, the only non-zero terms in 2 Re $\left\langle H u_{\alpha} \Psi_{0}, \widetilde{\Psi}_{0}^{\perp}\right\rangle$ are given by

$$
\begin{aligned}
& 2 \operatorname{Re}\left\langle H u_{\alpha} \Psi_{0}, \widetilde{\Psi}_{0}^{\perp}\right\rangle \\
& \quad=-4 \operatorname{Re}\left\langle P . P_{f} u_{\alpha} \Psi_{0}, \widetilde{\Psi}_{0}^{\perp}\right\rangle-4 \operatorname{Re}\left\langle P . A^{+} u_{\alpha} \Psi_{0}, \widetilde{\Psi}_{0}^{\perp}\right\rangle-4 \operatorname{Re}\left\langle P . A^{-} u_{\alpha} \Psi_{0}, \widetilde{\Psi}_{0}^{\perp}\right\rangle .
\end{aligned}
$$

$\diamond$ The first term on the right-hand side of (109) is estimated with similar arguments as in Lemma 7.2, and using $\left\|\Delta_{*}^{u_{\alpha}}\right\|^{2}=\mathcal{O}\left(\alpha^{3}\right)$ (Lemma C.1) and $\left\|\Delta_{*}^{u_{\alpha}}\right\|_{*}^{2}=\mathcal{O}\left(\alpha^{4}\right)([7$, Theorem 3.2]). We obtain

$$
-4 \operatorname{Re}\left\langle P . P_{f} u_{\alpha} \Psi_{0}, \widetilde{\Psi}_{0}^{\perp}\right\rangle=-\frac{2}{3} \alpha^{4} \sum_{i=1}^{3}\left\langle P_{f}^{i} \Phi_{*}^{2},\left(H_{f}+P_{f}^{2}\right) W_{i}\right\rangle+\mathcal{O}\left(\alpha^{5} \sqrt{\log \alpha^{-1}}\right) .
$$

$\diamond$ The second and third terms on the right-hand side of (109) are estimated as in Lemma 7.3, and using again $\left\|\Delta_{*}^{u_{\alpha}}\right\|^{2}=\mathcal{O}\left(\alpha^{2}\right)$ and $\left\|\Delta_{*}^{u_{\alpha}}\right\|_{*}^{2}=$ $\mathcal{O}\left(\alpha^{4}\right)$. This yields

$$
-4 \operatorname{Re}\left\langle P . A^{+} u_{\alpha} \Psi_{0}, \widetilde{\Psi}_{0}^{\perp}\right\rangle=-2\left\|\Phi_{\sharp}^{u_{\alpha}}\right\|_{\sharp}^{2}+o\left(\alpha^{5} \log \alpha^{-1}\right),
$$

and

$$
\begin{aligned}
& -4 \operatorname{Re}\left\langle P . A^{-} u_{\alpha} \Psi_{0}, \widetilde{\Psi}_{0}^{\perp}\right\rangle \\
& \quad=-\frac{2}{3} \alpha^{4} \sum_{i=1}^{3}\left\langle\left(A^{-}\right)^{i} \Phi_{*}^{2},\left(H_{f}+P_{f}^{2}\right)^{-1}\left(A^{+}\right)^{i} \Omega_{f}\right\rangle+\mathcal{O}\left(\alpha^{5} \sqrt{\log \alpha^{-1}}\right) .
\end{aligned}
$$

- Next, we estimate the third term on the right-hand side of (106). For that sake, we also use the decomposition (107) for $H$. 
$\diamond$ For the term involving $h_{\alpha}$, using $\left\|\left(h_{\alpha}+e_{0}\right) \pi^{0} \widetilde{\Psi}_{0}^{\perp}\right\|=\mathcal{O}\left(\alpha^{3}\right)$ (since $\left.\left\|P . A^{-} \Phi_{*}^{u_{\alpha}}\right\|=\mathcal{O}\left(\alpha^{\frac{5}{2}}\right)\right)$, and $\left\|\left(h_{\alpha}+e_{0}\right) \frac{\partial u_{\alpha}}{\partial x_{i}}\right\|=\mathcal{O}\left(\alpha^{3}\right)$, we directly obtain

$$
\begin{aligned}
\left\langle h_{\alpha} \widetilde{\Psi}_{0}^{\perp}, \widetilde{\Psi}_{0}^{\perp}\right\rangle= & \left\langle\left(h_{\alpha}+e_{0}\right) \widetilde{\Psi}_{0}^{\perp}, \widetilde{\Psi}_{0}^{\perp}\right\rangle-e_{0}\left\|\widetilde{\Psi}_{0}^{\perp}\right\|^{2} \\
= & 4 \alpha\left\|\left(h_{\alpha}+e_{0}\right)^{-\frac{1}{2}} Q_{\alpha}^{\perp} P . A^{-} \Phi_{*}^{u_{\alpha}}\right\|^{2} \\
& +\left\langle\left(h_{\alpha}+e_{0}\right) \Phi_{\sharp}^{u_{\alpha}}, \Phi_{\sharp}^{u_{\alpha}}\right\rangle-e_{0}\left\|\widetilde{\Psi}_{0}^{\perp}\right\|^{2}+\mathcal{O}\left(\alpha^{5}\right) .
\end{aligned}
$$

$\diamond$ For the term with $H_{f}+P_{f}^{2}$, we use the estimate (222) of Lemma C.6, and the $\langle\cdot, \cdot\rangle_{*}$-orthogonality (see (206) of Lemma C.2) of the two vectors $\alpha \Phi_{*}^{2} \pi^{0} g$ and $\sum_{i=1}^{3} 2 \alpha\left(H_{f}+P_{f}^{2}\right)^{-1} W_{i} \frac{\partial u_{\alpha}}{\partial x_{i}}$ that occur in $\pi^{2} \widetilde{\Psi}_{0}^{\perp}$. We therefore obtain

$$
\begin{aligned}
\left\langle\left(H_{f}+P_{f}^{2}\right) \widetilde{\Psi}_{0}^{\perp}, \widetilde{\Psi}_{0}^{\perp}\right\rangle= & \left\langle\left(H_{f}+P_{f}^{2}\right) \Phi_{\sharp}^{u_{\alpha}}, \Phi_{\sharp}^{u_{\alpha}}\right\rangle+\alpha^{2}\left\|\Phi_{*}^{2} \pi^{0} \widetilde{\Psi}_{0}^{\perp}\right\|_{*}^{2} \\
& +\left\|\sum_{i=1}^{3} 2 \alpha\left(H_{f}+P_{f}^{2}\right)^{-1} W_{i} \frac{\partial u_{\alpha}}{\partial x_{i}}\right\|_{*}^{2} \\
& +\left\|\Phi_{*}^{2}\right\|_{*}^{2}\left\|\Phi_{\sharp}^{u_{\alpha}}\right\|^{2}+o\left(\alpha^{5} \log \alpha^{-1}\right) .
\end{aligned}
$$

$\diamond \quad$ Using the symmetry of $u_{\alpha}$, all terms in $\operatorname{Re}\left\langle P . P_{f} \widetilde{\Psi}_{0}^{\perp}, \widetilde{\Psi}_{0}^{\perp}\right\rangle$ are zero, except the expression $\operatorname{Re}\left\langle P . P_{f} \pi^{2} \widetilde{\Psi}_{0}^{\perp}, \pi^{2} \widetilde{\Psi}_{0}^{\perp}\right\rangle$, which is estimated as follows:

$$
\begin{aligned}
& \operatorname{Re}\left\langle P . P_{f} \pi^{2} \widetilde{\Psi}_{0}^{\perp}, \pi^{2} \widetilde{\Psi}_{0}^{\perp}\right\rangle \\
& =2 \operatorname{Re}\left\langle P . P_{f} \alpha \Phi_{*}^{2} \pi^{0} \widetilde{\Psi}_{0}^{\perp}, \sum_{i=1}^{3} 2 \alpha\left(H_{f}+P_{f}^{2}\right)^{-1} W_{i} \frac{\partial u_{\alpha}}{\partial x_{i}}\right\rangle=\mathcal{O}\left(\alpha^{5}\right),
\end{aligned}
$$

where we used Lemma C.2 in the first equality to prove that only the crossed term remains. Therefore, we obtain

$$
-2 \operatorname{Re}\left\langle P . P_{f} \widetilde{\Psi}_{0}^{\perp}, \widetilde{\Psi}_{0}^{\perp}\right\rangle=\mathcal{O}\left(\alpha^{5}\right) .
$$

$\diamond$ The terms involving $-2 \alpha^{\frac{1}{2}} \operatorname{Re}\left\langle P . A(0) \widetilde{\Psi}_{0}^{\perp}, \widetilde{\Psi}_{0}^{\perp}\right\rangle$ is estimated as in the proof of Lemma B.6. This yields

$$
\begin{aligned}
- & 2 \alpha^{\frac{1}{2}}\left\langle P \cdot A(0) \widetilde{\Psi}_{0}^{\perp}, \widetilde{\Psi}_{0}^{\perp}\right\rangle=-4 \operatorname{Re} \alpha^{\frac{1}{2}}\left\langle P \cdot A^{+} \widetilde{\Psi}_{0}^{\perp}, \widetilde{\Psi}_{0}^{\perp}\right\rangle \\
& =-4 \operatorname{Re}\left\langle P \cdot A^{+} \pi^{0} \widetilde{\Psi}_{0}^{\perp}, \pi^{1} \widetilde{\Psi}_{0}^{\perp}\right\rangle+\mathcal{O}\left(\alpha^{5} \sqrt{\log \alpha^{-1}}\right) \\
& =-8 \alpha\left\|\left(h_{\alpha}+e_{0}\right)^{-\frac{1}{2}} Q_{\alpha}^{\perp} P \cdot A^{-} \Phi_{*}^{u_{\alpha}}\right\|^{2}+\mathcal{O}\left(\alpha^{5} \sqrt{\log \alpha^{-1}}\right) .
\end{aligned}
$$

$\diamond$ For $2 \alpha^{\frac{1}{2}} \operatorname{Re}\left\langle P_{f} \cdot A(0) \widetilde{\Psi}_{0}^{\perp}, \widetilde{\Psi}_{0}^{\perp}\right\rangle$, we proceed as in the proof of Lemma B.7, and obtain

$$
\begin{aligned}
& 2 \alpha^{\frac{1}{2}}\left\langle P_{f} \cdot A(0) \widetilde{\Psi}_{0}^{\perp}, \widetilde{\Psi}_{0}^{\perp}\right\rangle \\
& \quad=4 \operatorname{Re} \alpha^{\frac{1}{2}}\left\langle P_{f} \cdot A^{-} \widetilde{\Psi}_{0}^{\perp}, \widetilde{\Psi}_{0}^{\perp}\right\rangle \\
& \quad=4 \alpha^{\frac{1}{2}}\left\langle P_{f} \cdot A^{-} \alpha \sum_{i=1}^{3} 2\left(H_{f}+P_{f}^{2}\right)^{-1} W_{i} \frac{\partial u_{\alpha}}{\partial x_{i}}, \Phi_{*}^{u_{\alpha}}\right\rangle+\mathcal{O}\left(\alpha^{5} \sqrt{\log \alpha^{-1}}\right) .
\end{aligned}
$$


$\diamond$ Using the symmetry of $u_{\alpha}$ and $\pi^{0} \widetilde{\Psi}_{0}^{\perp}$, the term $2 \alpha \operatorname{Re}\left\langle A^{-} \cdot A^{-} \widetilde{\Psi}_{0}^{\perp}, \widetilde{\Psi}_{0}^{\perp}\right\rangle$ is estimated as follows:

$$
\begin{aligned}
& 2 \alpha \operatorname{Re}\left\langle A^{-} \cdot A^{-} \widetilde{\Psi}_{0}^{\perp}, \widetilde{\Psi}_{0}^{\perp}\right\rangle \\
&=2 \alpha \operatorname{Re}\left\langle A^{-} \cdot A^{-}\left(\alpha \Phi_{*}^{2} \pi^{0} \widetilde{\Psi}_{0}^{\perp}+\sum_{i=1}^{3} 2 \alpha\left(H_{f}+P_{f}^{2}\right)^{-1} W_{i} \frac{\partial u_{\alpha}}{\partial x_{i}}\right), \pi^{0} \widetilde{\Psi}_{0}^{\perp}\right\rangle \\
&+2 \alpha \operatorname{Re}\left\langle A^{-} \cdot A^{-}\left(-\alpha\left(H_{f}+P_{f}^{2}\right)^{-1} A^{+} \cdot A^{+} \Phi_{\sharp}^{u_{\alpha}}\right), \Phi_{\sharp}^{u_{\alpha}}\right\rangle \\
&=-2 \alpha^{2}\left\|\pi^{0} \widetilde{\Psi}_{0}^{\perp}\right\|^{2}\left\|\Phi_{*}^{2}\right\|_{*}^{2}-2 \alpha^{2}\left\|\left(H_{f}+P_{f}^{2}\right)^{-1} A^{+} \cdot A^{+} \Phi_{\sharp}^{u_{\alpha}}\right\|^{2} \\
&=-2 \alpha^{2}\left\|\pi^{0} \widetilde{\Psi}_{0}^{\perp}\right\|^{2}\left\|\Phi_{*}^{2}\right\|_{*}^{2}-2 \alpha^{2}\left\|\Phi_{*}^{2}\right\|_{*}^{2}\left\|\Phi_{\sharp}^{u_{\alpha}}\right\|^{2}+o\left(\alpha^{5} \log \alpha^{-1}\right),
\end{aligned}
$$

where in the last inequality we used (222) of Lemma C.6.

$\diamond$ Finally, a straightforward computation yields

$$
2 \alpha \operatorname{Re}\left\langle A^{-} . A^{+} \widetilde{\Psi}_{0}^{\perp}, \widetilde{\Psi}_{0}^{\perp}\right\rangle=2 \alpha\left\|A^{-} \Phi_{\sharp}^{u_{\alpha}}\right\|^{2}+\mathcal{O}\left(\alpha^{5}\right)=2 \alpha\left\|A^{-} \Phi_{*}^{u_{\alpha}}\right\|^{2}+\mathcal{O}\left(\alpha^{5}\right),
$$

where in the last equality, we used Lemma C.5.

- Before collecting (109)-(119), we show that gathering some terms yield simpler expressions. Namely, we have

$$
\begin{aligned}
& -\frac{2}{3} \alpha^{4} \sum_{i=1}^{3}\left\langle P_{f}^{i} \Phi_{*}^{2},\left(H_{f}+P_{f}^{2}\right) W_{i}\right\rangle \\
& \quad+4 \alpha^{\frac{1}{2}}\left\langle P_{f} \cdot A^{-} \alpha \sum_{i=1}^{3} 2\left(H_{f}+P_{f}^{2}\right)^{-1} W_{i} \frac{\partial u_{\alpha}}{\partial x_{i}}, \Phi_{*}^{u_{\alpha}}\right\rangle \\
& \quad+\left\|\sum_{i=1}^{3} 2 \alpha\left(H_{f}+P_{f}^{2}\right)^{-1} W_{i} \frac{\partial u_{\alpha}}{\partial x_{i}}\right\|_{*}^{2}=-\frac{1}{3} \alpha^{4} \sum_{i=1}^{3}\left\|\left(H_{f}+P_{f}^{2}\right)^{-1} W_{i}\right\|_{*}^{2} \cdot
\end{aligned}
$$

We also have, using $-\alpha^{2}\left\|\Phi_{*}^{2}\right\|_{*}^{2}=\Sigma_{0}+\mathcal{O}\left(\alpha^{3}\right)$ (see, e.g., [7])

$$
\begin{aligned}
& \left(\Sigma_{0}-e_{0}\right)\left\|\Psi_{0}\right\|^{2}-e_{0}\left\|\widetilde{\Psi}_{0}^{\perp}\right\|^{2}-\alpha^{2}\left\|\Phi_{*}^{2}\right\|_{*}^{2}\left\|\pi^{0} \widetilde{\Psi}_{0}^{\perp}\right\|^{2}-\alpha^{2}\left\|\Phi_{*}^{2}\right\|_{*}^{2}\left\|\Phi_{\sharp}^{u_{\alpha}}\right\|^{2} \\
& \quad=\left(\Sigma_{0}-e_{0}\right)\left(\left\|\Psi_{0}\right\|^{2}+\left\|\widetilde{\Psi}_{0}^{\perp}\right\|^{2}\right)+\mathcal{O}\left(\alpha^{5}\right) .
\end{aligned}
$$

Therefore, collecting (109)-(119), and using the two equalities (120)-(121), we obtain

$$
\begin{aligned}
& \left\langle H\left(u_{\alpha} \Psi_{0}+\widetilde{\Psi}_{0}^{\perp}\right), u_{\alpha} \Psi_{0}+\widetilde{\Psi}_{0}^{\perp}\right\rangle \\
& =\left(\Sigma_{0}-e_{0}\right)\left(\left\|\Psi_{0}\right\|^{2}+\left\|\widetilde{\Psi}_{0}^{\perp}\right\|^{2}\right)-\frac{1}{3} \alpha^{4} \sum_{i=1}^{3}\left\|\left(H_{f}+P_{f}^{2}\right)^{-1} W_{i}\right\|_{*}^{2} \\
& \quad-\frac{2}{3} \alpha^{4} \sum_{i=1}^{3}\left\langle\left(A^{-}\right)^{i} \Phi_{*}^{2},\left(H_{f}+P_{f}^{2}\right)^{-1}\left(A^{+}\right)^{i} \Omega_{f}\right\rangle-\left\|\Phi_{\sharp}^{u_{\alpha}}\right\|_{\sharp}^{2} \\
& \quad-4 \alpha\left\|\left(h_{\alpha}+e_{0}\right)^{-\frac{1}{2}} Q_{\alpha}^{\perp} P . A^{-} \Phi_{*}^{u_{\alpha}}\right\|^{2}+2 \alpha\left\|A^{-} \Phi_{*}^{u_{\alpha}}\right\|^{2}+o\left(\alpha^{5} \log \alpha^{-1}\right) .
\end{aligned}
$$


With the definition $e^{(1)}, e^{(2)}$, and $e^{(3)}$, of Theorem 2.1 this expression can be rewritten as

$$
\begin{aligned}
& \left\langle\left(H-\Sigma_{0}+e_{0}\right) \widetilde{\Phi}^{\text {trial }}, \widetilde{\Phi}^{\text {trial }}\right\rangle \\
& \quad=e^{(1)} \alpha^{3}+e^{(2)} \alpha^{4}+e^{(3)} \alpha^{5} \log \alpha^{-1}+o\left(\alpha^{5} \log \alpha^{-1}\right) .
\end{aligned}
$$

Using Lemma C.1 yields $\left\|\Psi_{0}\right\|^{2}=1+\mathcal{O}\left(\alpha^{2}\right)$, which implies, due to the orthogonality of $\widetilde{\Psi}_{0}^{\perp}$ and $u_{\alpha}$ in $L^{2}\left(\mathbb{R}^{3}, \mathrm{~d} x\right)$,

$$
\left\|\widetilde{\Phi}^{\text {trial }}\right\|^{2}=\left\|u_{\alpha}\right\|^{2}\left\|\Psi_{0}\right\|^{2}+\left\|\widetilde{\Psi}_{0}^{\perp}\right\|^{2}=1+\mathcal{O}\left(\alpha^{2}\right) .
$$

Therefore, together with (122), this gives

$$
\begin{aligned}
& \frac{\left\langle\left(H-\Sigma_{0}+e_{0}\right) \widetilde{\Phi}^{\text {trial }}, \widetilde{\Phi}^{\text {trial }}\right\rangle}{\left\|\widetilde{\Phi}^{\text {trial }}\right\|^{2}} \\
& \quad=e^{(1)} \alpha^{3}+e^{(2)} \alpha^{4}+e^{(3)} \alpha^{5} \log \alpha^{-1}+o\left(\alpha^{5} \log \alpha^{-1}\right) .
\end{aligned}
$$

which concludes the proof of the lower bound in Theorem 2.1.

\section{Acknowledgements}

J.-M. B. thanks V. Bach for fruitful discussions. The authors gratefully acknowledge financial support from the following institutions: The European Union through the IHP network Analysis and Quantum HPRN-CT-200200277 (J.-M. B., T. C., and S. V.), the French Ministry of Research through the project ANR HAM-MARK No. ANR-09-BLAN-0098-01 (J.-M. B.), and the DFG grant WE 1964/2 (S. V.). The work of T.C. was supported by NSF grant DMS-0704031/DMS-0940145.

\section{Appendix A. Proof of Proposition 6.1}

In this Appendix, we provide proofs of results that have a high level of technicality. To begin with, we establish Proposition 6.1.

Lemma A.1. The following holds:

$$
\begin{aligned}
& -4 \operatorname{Re}\left\langle P . P_{f} u_{\alpha} \Psi^{u_{\alpha}}, \Psi^{\perp}\right\rangle \geq-\frac{4}{3} \alpha^{2} \operatorname{Re} \sum_{i=1}^{3} \overline{\kappa_{2, i}}\left\|\nabla u_{\alpha}\right\|^{2}\left\langle\left(H_{f}+P_{f}^{2}\right)^{-1} P_{f}^{i} \Phi_{*}^{2}, W_{i}\right\rangle \\
& -4 \alpha \operatorname{Re}\left\langle\nabla u_{\alpha} \cdot P_{f} \Phi_{*}^{2}, \pi^{2} \Psi_{2}^{\perp}\right\rangle-\epsilon\left\|H_{f}^{\frac{1}{2}} \Psi_{2}^{\perp}\right\|^{2}-\epsilon \alpha^{5}\left|\kappa_{3}\right|^{2}+\mathcal{O}\left(\alpha^{5}\right)
\end{aligned}
$$

Proof. For $n \neq 2,3$, with the estimates from the proof of Lemma 7.2 and using that due to Theorem 5.2 we have

$$
\left\|H_{f}^{\frac{1}{2}} \Delta_{*}^{u_{\alpha}}\right\|^{2}=\mathcal{O}\left(\alpha^{4}\right),\left|\eta_{1}\right|=\mathcal{O}(1), \quad \text { and } \quad\left|\kappa_{1}\right|=\mathcal{O}(1),
$$

and since $\pi^{1} \Delta_{\sharp}^{\perp}=\pi^{1} \Psi_{2}^{\perp}$ and $\pi^{n \geq 4} \Delta_{\sharp}^{\perp}=\pi^{n \geq 4} \Psi_{2}^{\perp}$, we obtain

$$
\sum_{n \neq 2,3}-4 \operatorname{Re}\left\langle P . P_{f} u_{\alpha} \pi^{n} \Psi^{u_{\alpha}}, \pi^{n} \Psi^{\perp}\right\rangle \geq-\epsilon\left\|H_{f}^{\frac{1}{2}} \Psi_{2}^{\perp}\right\|^{2}+\mathcal{O}\left(\alpha^{5}\right) .
$$


For $n=2$,

$$
\begin{gathered}
-4 \operatorname{Re}\left\langle\nabla u_{\alpha} \cdot\left(\alpha \eta_{2} P_{f} \Phi_{*}^{2}+P_{f} \pi^{2} \Delta_{*}^{u_{\alpha}}\right), \pi^{2} \Psi^{\perp}\right\rangle \geq-4 \operatorname{Re}\left\langle\nabla u_{\alpha} . \alpha \eta_{2} P_{f} \Phi_{*}^{2}, \pi^{2} \Psi_{1}^{\perp}\right\rangle \\
-4 \operatorname{Re}\left\langle\nabla u_{\alpha} \cdot \alpha \eta_{2} P_{f} \Phi_{*}^{2}, \pi^{2} \Psi_{2}^{\perp}\right\rangle-c \alpha\left\|H_{f}^{\frac{1}{2}} \pi^{2} \Delta_{*}^{u_{\alpha}}\right\|^{2}-c \alpha\left\|H_{f}^{\frac{1}{2}} \pi^{2} \Psi^{\perp}\right\|^{2} .
\end{gathered}
$$

Using Theorem 5.2, the last two terms on the right-hand side of (125) can be estimated by $\mathcal{O}\left(\alpha^{5}\right)$. For the first term on the right-hand side of (125), using from Lemma C.2 that $\left\langle P_{f}^{i} \Phi_{*}^{2}, \Phi_{*}^{2}\right\rangle=0$, from Theorem 5.2 that $\eta_{2}=1+\mathcal{O}(\alpha)$, and from Lemma 6.1 that $\kappa_{2, i}=\mathcal{O}(1)$, holds

$$
\begin{aligned}
& -4 \alpha \operatorname{Re}\left\langle\nabla u_{\alpha} \cdot \eta_{2} P_{f} \Phi_{*}^{2}, \pi^{2} \Psi_{1}^{\perp}\right\rangle \\
& =-4 \operatorname{Re} \alpha\left\langle\nabla u_{\alpha} \cdot \eta_{2} P_{f} \Phi_{*}^{2}, \alpha \kappa_{2} \Phi_{*}^{2} \pi^{0} \Psi^{\perp}\right\rangle \\
& -4 \operatorname{Re} \alpha\left\langle\nabla u_{\alpha} \cdot \eta_{2} P_{f} \Phi_{*}^{2}, \sum_{i} \alpha \kappa_{2, i}\left(H_{f}+P_{f}^{2}\right)^{-1} W_{i} \frac{\partial u_{\alpha}}{\partial x_{i}}\right\rangle \\
& =-\frac{4}{3} \operatorname{Re} \alpha^{2}\left\|\nabla u_{\alpha}\right\|^{2} \sum_{i} \overline{\kappa_{2, i}}\left\langle\left(H_{f}+P_{f}^{2}\right)^{-1} P_{f}^{i} \Phi_{*}^{2}, W_{i}\right\rangle \\
& =-\frac{1}{3} \alpha^{4} \operatorname{Re} \sum_{i=1}^{3} \overline{\kappa_{2, i}}\left\langle\left(H_{f}+P_{f}^{2}\right)^{-1} P_{f}^{i} \Phi_{*}^{2}, W_{i}\right\rangle .
\end{aligned}
$$

We also used that $\left\langle\frac{\partial u_{\alpha}}{\partial x_{i}}, \frac{\partial u_{\alpha}}{\partial x_{j}}\right\rangle=0$ for $i \neq j$ and $\left\|\frac{\partial u_{\alpha}}{\partial x_{i}}\right\|=\left\|\frac{\partial u_{\alpha}}{\partial x_{j}}\right\|$ for all $i$ and $j$.

Finally, the second term on the right-hand side of (125) gives the second term on the right-hand side of (123) plus $\mathcal{O}\left(\alpha^{5}\right)$, using from Theorem 5.2 that $\left|\eta_{2}-1\right|^{2}=\mathcal{O}\left(\alpha^{2}\right)$ and $\left\|H_{f}^{\frac{1}{2}} \pi^{2} \Psi_{2}^{\perp}\right\|=\mathcal{O}\left(\alpha^{2}\right)$.

To complete the proof, we shall estimate now the term for $n=3$,

$$
\begin{aligned}
& 4 \operatorname{Re}\left\langle P . P_{f} u_{\alpha} \pi^{3} \Psi^{u_{\alpha}}, \pi^{3} \Psi^{\perp}\right\rangle=4 \operatorname{Re} \alpha^{\frac{3}{2}} 2 \eta_{3}\left\langle P . P_{f} u_{\alpha} \Phi_{*}^{3}, \pi^{3} \Psi_{1}^{\perp}\right\rangle \\
& \quad+4 \operatorname{Re} \alpha^{\frac{3}{2}} 2 \eta_{3}\left\langle P . P_{f} u_{\alpha} \Phi_{*}^{3}, \pi^{3} \Psi_{2}^{\perp}\right\rangle+4 \operatorname{Re}\left\langle P . P_{f} u_{\alpha} \pi^{3} \Delta_{*}^{u_{\alpha}}, \pi^{3} \Psi^{\perp}\right\rangle .
\end{aligned}
$$

The inequalities $\left\|H_{f}^{\frac{1}{2}} \Delta_{*}^{u_{\alpha}}\right\| \leq c \alpha^{2}$ and $\left\|H_{f}^{\frac{1}{2}} \pi^{3} \Psi^{\perp}\right\| \leq c \alpha^{2}$ (see Theorem 5.2) imply that the last term on the right-hand side of $(127)$ is $\mathcal{O}\left(\alpha^{5}\right)$. For the second term on the right-hand side of (127) holds

$$
\operatorname{Re} \alpha^{\frac{3}{2}} \eta_{3}\left\langle P . P_{f} u_{\alpha} \Phi_{*}^{3}, \pi^{3} \Psi_{2}^{\perp}\right\rangle \geq-\epsilon\left\|H_{f}^{\frac{1}{2}} \pi^{3} \Psi_{2}^{\perp}\right\|^{2}+\mathcal{O}\left(\alpha^{5}\right)
$$

since from Theorem 5.2 we have $\eta_{3}=\mathcal{O}(1)$.

Finally to estimate the first term on the right-hand side of (127), we note that

$$
\left|k_{1}\right|^{-\frac{1}{6}}\left|k_{2}\right|^{-\frac{1}{6}}\left|k_{3}\right|^{-\frac{1}{6}} \Phi_{*}^{3}\left(k_{1}, k_{2}, k_{3}\right) \in L^{2}\left(\mathbb{R}^{9}, \mathbb{C}^{6}\right),
$$

and from Lemma C.6,

$$
\left\|\left|k_{1}\right|^{\frac{1}{6}}\left|k_{2}\right|^{\frac{1}{6}}\left|k_{3}\right|^{\frac{1}{6}}\left(H_{f}+P_{f}^{2}\right)^{-1} A^{+} \cdot A^{+} \Phi_{\sharp}^{u_{\alpha}}\right\|^{2}=\mathcal{O}\left(\alpha^{3}\right) .
$$

This implies, using again $\left|\eta_{3}\right|=\mathcal{O}(1)$, and the explicit expression of $\pi^{3} \Psi_{1}^{\perp}$ $\left|\alpha^{\frac{3}{2}} 2 \eta_{3}\left\langle P . P_{f} u_{\alpha} \Phi_{*}^{3}, \pi^{3} \Psi_{1}^{\perp}\right\rangle\right| \leq c \alpha^{\frac{5}{2}}\left|\kappa_{3}\right| \alpha^{\frac{3}{2}}\left|\eta_{3}\right|\left\|P u_{\alpha}\right\| \leq \epsilon \alpha^{5}\left|\kappa_{3}\right|^{2}+\mathcal{O}\left(\alpha^{5}\right)$.

Collecting (124)-(129) concludes the proof. 
Lemma A.2. The following estimate holds:

$$
\begin{aligned}
& -4 \sqrt{\alpha} \operatorname{Re}\left\langle P . A^{+} u_{\alpha} \Psi^{u_{\alpha}}, \Psi^{\perp}\right\rangle \\
& =-2 \operatorname{Re} \overline{\kappa_{1}} \pi^{0} \Psi^{u_{\alpha}}\left\|\Phi_{\sharp}^{u_{\alpha}}\right\|_{\sharp}^{2}-\frac{1}{4} M\left[\Psi_{2}^{\perp}\right]-\alpha^{5}\left|\kappa_{3}\right|^{2}+\mathcal{O}\left(\alpha^{5}\right),
\end{aligned}
$$

Proof. Obviously

$$
\begin{aligned}
& -4 \operatorname{Re} \alpha^{\frac{1}{2}}\left\langle P . A^{+} u_{\alpha} \Psi^{u_{\alpha}}, \Psi^{\perp}\right\rangle \\
& \quad=-4 \operatorname{Re} \alpha^{\frac{1}{2}}\left\langle P . A^{+} u_{\alpha}\left(\pi^{0} \Psi^{u_{\alpha}}+2 \eta_{1} \alpha^{\frac{3}{2}} \Phi_{*}^{1}+\eta_{2} \alpha \Phi_{*}^{2}+2 \eta_{3} \alpha^{\frac{3}{2}} \Phi_{*}^{3}+\Delta_{*}^{u_{\alpha}}\right), \Psi^{\perp}\right\rangle .
\end{aligned}
$$

$\diamond$ Step 1. From (131), let us first estimate the term

$$
-4 \operatorname{Re} \alpha^{\frac{1}{2}}\left\langle P . A^{+} u_{\alpha} \Delta_{*}^{u_{\alpha}}, \Psi^{\perp}\right\rangle=-4 \alpha^{\frac{1}{2}} \operatorname{Re} \sum_{n=0}^{\infty}\left\langle P . A^{+} \pi^{n} u_{\alpha} \Delta_{*}^{u_{\alpha}}, \pi^{n+1} \Psi^{\perp}\right\rangle .
$$

For $n=0$, the corresponding term vanishes since $\pi^{0} \Delta_{*}^{u_{\alpha}}=0$.

For $n>2$, we can use (62) where the term $\mathcal{O}\left(\alpha^{5} \log \alpha^{-1}\right)$ can be replaced with $\mathcal{O}\left(\alpha^{5}\right)$ because we know from Theorem 5.2 that $\left\|\Delta_{*}^{u_{\alpha}}\right\|^{2}=\mathcal{O}\left(\alpha^{\frac{33}{16}}\right)$.

For $n=1$, we have

$$
\begin{aligned}
& \left|4 \alpha^{\frac{1}{2}} \operatorname{Re}\left\langle P . A^{+} \pi^{1} u_{\alpha} \Delta_{*}^{u_{\alpha}}, \pi^{2} \Psi_{1}^{\perp}+\pi^{2} \Psi_{2}^{\perp}\right\rangle\right| \leq c \alpha^{3}\left\|\pi^{1} \Delta_{*}^{u_{\alpha}}\right\|^{2}+\epsilon\left\|H_{f}^{\frac{1}{2}} \pi^{2} \Psi_{2}^{\perp}\right\|^{2} \\
& \quad+\left|4 \alpha^{\frac{1}{2}}\left\langle\nabla u_{\alpha} \pi^{1} \Delta_{*}^{u_{\alpha}}, A^{-}\left(\alpha \kappa_{2} \Phi_{*}^{2} \pi^{0} \Psi_{1}^{\perp}+\alpha \sum_{i=1}^{3} \kappa_{2, i}\left(H_{f}+P_{f}^{2}\right)^{-1} W_{i} \frac{\partial u_{\alpha}}{\partial x_{i}}\right)\right\rangle\right| .
\end{aligned}
$$

To estimate the last term on the right-hand side we note that $H_{f}^{-\frac{1}{2}} A^{-} \Phi_{*}^{2} \in L^{2}$ and $H_{f}^{-\frac{1}{2}} A^{-}\left(H_{f}+P_{f}^{2}\right)^{-1} W_{i} \in L^{2}$ which thus gives for this term the bound

$$
c \alpha\left\|H_{f}^{\frac{1}{2}} \Delta_{*}^{u_{\alpha}}\right\|^{2}+\epsilon \alpha^{4}\left|\kappa_{2}\right|^{2}\left\|\pi^{0} \Psi_{1}^{\perp}\right\|^{2}+\epsilon \alpha^{6} \sum_{i}\left|\kappa_{2, i}\right|^{2}=\mathcal{O}\left(\alpha^{5}\right),
$$

using Theorem 5.2 and Lemma 6.1. The inequalities (133) and (134) imply

$$
\left|\operatorname{Re} \alpha^{\frac{1}{2}}\left\langle P . A^{+} \pi^{1} u_{\alpha} \Delta_{*}^{u_{\alpha}}, \pi^{2} \Psi_{1}^{\perp}+\pi^{2} \Psi_{2}^{\perp}\right\rangle\right| \leq \epsilon\left\|H_{f}^{\frac{1}{2}} \pi^{2} \Psi_{2}^{\perp}\right\|^{2}+\mathcal{O}\left(\alpha^{5}\right) .
$$

To complete the estimate of the term $4 \alpha^{\frac{1}{2}} \operatorname{Re}\left\langle P . A^{+} u_{\alpha} \Delta_{*}^{u_{\alpha}}, \Psi^{\perp}\right\rangle$ we have to estimate the term for $n=2$ in (132), namely $4 \operatorname{Re} \alpha^{\frac{1}{2}}\left\langle P . A^{+} u_{\alpha} \pi^{2} \Delta_{*}^{u_{\alpha}}, \pi^{3} \Psi_{1}^{\perp}+\right.$ $\left.\pi^{3} \Psi \frac{\perp}{2}\right\rangle$. Obviously,

$$
\left|\operatorname{Re} \alpha^{\frac{1}{2}}\left\langle P . A^{+} u_{\alpha} \pi^{2} \Delta_{*}^{u_{\alpha}}, \pi^{3} \Psi_{2}^{\perp}\right\rangle\right| \leq \epsilon\left\|H_{f}^{\frac{1}{2}} \Psi_{2}^{\perp}\right\|^{2}+\mathcal{O}\left(\alpha^{5}\right) .
$$

For the term involving $\pi^{3} \Psi_{1}^{\perp}$ we have

$$
\begin{aligned}
& \left|\operatorname{Re} \alpha^{\frac{1}{2}}\left\langle P u_{\alpha} \pi^{2} \Delta_{*}^{u_{\alpha}}, \alpha \kappa_{3} A^{-}\left(H_{f}+P_{f}^{2}\right)^{-1} A^{+} \cdot A^{+} \Phi_{\sharp}^{u_{\alpha}}\right\rangle\right| \\
& \quad \leq c \alpha^{3-\frac{1}{16}}\left\|\pi^{2} \Delta_{*}^{u_{\alpha}}\right\|^{2}+\epsilon\left|\kappa_{3}\right|^{2} \alpha^{2+\frac{1}{16}}\left\|\Phi_{\sharp}^{u_{\alpha}}\right\|^{2}=\left|\kappa_{3}\right|^{2} \alpha^{5}+\mathcal{O}\left(\alpha^{5}\right),
\end{aligned}
$$

using Theorem 5.2 and Lemma C.6. Collecting (132)-(137) yields

$$
\left|\operatorname{Re} \alpha^{\frac{1}{2}}\left\langle P . A^{+} u_{\alpha} \Delta_{*}^{u_{\alpha}}, g\right\rangle\right| \leq \epsilon\left\|H_{f}^{\frac{1}{2}} \Psi_{2}^{\perp}\right\|^{2}+\alpha^{5}\left|\kappa_{3}\right|^{2}+\mathcal{O}\left(\alpha^{5}\right) .
$$


$\diamond$ Step 2. We next estimate in (131) the term $-4 \operatorname{Re} \alpha^{\frac{1}{2}}\left\langle P . A^{+} u_{\alpha}\left(\pi^{0} \Psi^{u_{\alpha}}+\right.\right.$ $\left.\left.\alpha^{\frac{3}{2}} 2 \eta_{1} \Phi_{*}^{1}+\alpha \eta_{2} \Phi_{*}^{2}+\alpha^{\frac{3}{2}} 2 \eta_{3} \Phi_{*}^{3}\right), \Psi^{\perp}\right\rangle$. First using (62) yields

$$
-4 \operatorname{Re} \alpha^{\frac{1}{2}}\left\langle P . A^{+} u_{\alpha} \pi^{0} \Psi^{u_{\alpha}}, \Psi^{\perp}\right\rangle=-2 \operatorname{Re}\left(\overline{\kappa_{1}} \pi^{0} \Psi^{u_{\alpha}}\right)\left\|\Phi_{\sharp}^{u_{\alpha}}\right\|_{\sharp}^{2} .
$$

We also have, using Theorem 5.2

$$
\begin{aligned}
& \left|\alpha^{\frac{1}{2}}\left\langle P u_{\alpha}\left(\alpha^{\frac{3}{2}} 2 \eta_{1} \Phi_{*}^{1}+\alpha^{\frac{3}{2}} 2 \eta_{3} \Phi_{*}^{3}\right), A^{-} \Psi^{\perp}\right\rangle\right| \\
& \quad \leq \alpha\left\|H_{f}^{\frac{1}{2}} \pi^{2} \Psi^{\perp}\right\|^{2}+\alpha\left\|H_{f}^{\frac{1}{2}} \pi^{4} \Psi^{\perp}\right\|^{2}+\mathcal{O}\left(\alpha^{5}\right)=\mathcal{O}\left(\alpha^{5}\right),
\end{aligned}
$$

and

$$
\begin{aligned}
& \left|\alpha^{\frac{1}{2}}\left\langle P u_{\alpha} \alpha \eta_{2} \Phi_{*}^{2}, A^{-}\left(\pi^{3} \Psi_{1}^{\perp}+\pi^{3} \Psi_{2}^{\perp}\right)\right\rangle\right| \\
& \quad \leq \epsilon\left\|H_{f}^{\frac{1}{2}} \pi^{3} \Psi_{2}^{\perp}\right\|^{2}+\left|\alpha^{\frac{3}{2}}\left\langle P u_{\alpha} \eta_{2}\left|k_{1}\right|^{-\frac{1}{4}}\left|k_{2}\right|^{-\frac{1}{4}} \Phi_{*}^{2},\left|k_{1}\right|^{\frac{1}{4}}\left|k_{2}\right|^{\frac{1}{4}} A^{-} \pi^{3} \Psi_{1}^{\perp}\right\rangle\right|+\mathcal{O}\left(\alpha^{5}\right) \\
& \quad \leq \epsilon\left\|H_{f}^{\frac{1}{2}} \pi^{3} \Psi_{2}^{\perp}\right\|^{2}+\epsilon\left|\kappa_{3}\right|^{2} \alpha^{5}+\mathcal{O}\left(\alpha^{5}\right) .
\end{aligned}
$$

Here we used $\left|k_{1}\right|^{-\frac{1}{4}}\left|k_{2}\right|^{-\frac{1}{4}} \Phi_{*}^{2} \in L^{2}$ and $\|\left|k_{1}\right|^{\frac{1}{4}}\left|k_{2}\right|^{\frac{1}{4}} A^{-}\left(H_{f}+P_{f}^{2}\right)^{-1} A^{+} . A^{+}$ $\Phi_{\sharp}^{u_{\alpha}} \|^{2}=\mathcal{O}\left(\alpha^{3}\right)$ (see Lemma C.6).

Collecting (138)-(141) yields

$$
\begin{aligned}
& -4 \operatorname{Re} \alpha^{\frac{1}{2}}\left\langle P \cdot A^{+} u_{\alpha} \Psi^{u_{\alpha}}, \Psi^{\perp}\right\rangle \\
& \quad \geq-2 \operatorname{Re} \overline{\kappa_{1}} \pi^{0} \Psi^{u_{\alpha}}\left\|\Phi_{\sharp}^{u_{\alpha}}\right\|_{\sharp}^{2}-\epsilon\left\|H_{f}^{\frac{1}{2}} \Psi_{2}^{\perp}\right\|^{2}-\epsilon \alpha^{5}\left|\kappa_{3}\right|^{2}+\mathcal{O}\left(\alpha^{5}\right) .
\end{aligned}
$$

\section{Lemma A.3.}

$$
\begin{aligned}
-4 \sqrt{\alpha} & \operatorname{Re}\left\langle P . A^{-} u_{\alpha} \Psi^{u_{\alpha}}, \Psi^{\perp}\right\rangle \\
\geq & -\frac{2}{3} \alpha^{4} \operatorname{Re} \sum_{i=1}^{3}\left\langle\left(H_{f}+P_{f}^{2}\right)^{-\frac{1}{2}}\left(A^{-}\right)^{i} \Phi_{*}^{2},\left(H_{f}+P_{f}^{2}\right)^{-\frac{1}{2}}\left(A^{+}\right)^{i} \Omega_{f}\right\rangle \\
& \quad-\frac{1}{4} M\left[\Psi_{2}^{\perp}\right]-\epsilon \alpha^{2}\left\|\left(\Psi_{1}^{\perp}\right)^{a}\right\|^{2}-\epsilon \alpha^{5} \log \alpha^{-1}\left(\alpha\left|\kappa_{3}\right|^{2}+1\right)-\left|\kappa_{1}-1\right| c \alpha^{4}+\mathcal{O}\left(\alpha^{5}\right),
\end{aligned}
$$

where $\left(\Psi_{1}^{\perp}\right)^{a}(x):=\left(\pi^{0} \Psi_{1}^{\perp}(x)-\pi^{0} \Psi_{1}^{\perp}(-x)\right) / 2$ is the odd part of $\pi^{0} \Psi_{1}^{\perp}$.

Proof. Since from Lemma C.2 we have $\nabla u_{\alpha} \cdot A^{-} \Phi_{*}^{1}=0$, we have

$$
\begin{aligned}
& 4 \operatorname{Re} \alpha^{\frac{1}{2}}\left\langle P . A^{-} u_{\alpha} \Psi^{u_{\alpha}}, \Psi^{\perp}\right\rangle=4 \alpha^{\frac{3}{2}} \operatorname{Re} \eta_{2}\left\langle A^{-} \Phi_{*}^{2} \cdot P u_{\alpha}, \pi^{1} \Psi^{\perp}\right\rangle \\
& \quad+4 \alpha^{2} \operatorname{Re} 2 \eta_{3}\left\langle A^{-} \Phi_{*}^{3} \cdot P u_{\alpha}, \pi^{2} \Psi^{\perp}\right\rangle+4 \alpha^{\frac{1}{2}}\left\langle A^{-} \Delta_{*}^{u_{\alpha}} \cdot P u_{\alpha}, \Psi^{\perp}\right\rangle .
\end{aligned}
$$

For the first term on the right-hand side of (144) we have

$$
\begin{aligned}
& 4 \alpha^{\frac{3}{2}} \operatorname{Re} \eta_{2}\left\langle A^{-} \Phi_{*}^{2} \cdot P u_{\alpha}, \pi^{1} \Psi_{2}^{\perp}+\kappa_{1} \Phi_{\sharp}^{u_{\alpha}}\right\rangle \\
& \quad=4 \alpha^{\frac{3}{2}} \operatorname{Re} \eta_{2}\left\langle H_{f}^{-\frac{1}{2}} A^{-} \Phi_{*}^{2} \cdot P u_{\alpha}, H_{f}^{\frac{1}{2}} \pi^{1} \Psi_{2}^{\perp}\right\rangle+4 \alpha^{\frac{3}{2}} \operatorname{Re} \eta_{2}\left\langle A^{-} \Phi_{*}^{2} \cdot P u_{\alpha}, \kappa_{1} \Phi_{\sharp}^{u_{\alpha}}\right\rangle .
\end{aligned}
$$

The first term on the right-hand side of (145) is bounded from below by $-\epsilon\left\|H_{f}^{\frac{1}{2}} \pi^{1} \Psi_{2}^{\perp}\right\|^{2}+\mathcal{O}\left(\alpha^{5}\right)$. 
Applying Lemma C.5, we can replace $\Phi_{\sharp}^{u_{\alpha}}$ in the second term of the right-hand side of (145) by $\Phi_{*}^{u_{\alpha}}$, at the expense of $\mathcal{O}\left(\alpha^{5}\right)$. More precisely,

$$
\begin{aligned}
& \left|\alpha^{\frac{3}{2}}\left\langle\eta_{2} A^{-} \Phi_{*}^{2} \cdot P u_{\alpha}, \kappa_{1}\left(\Phi_{\sharp}^{u_{\alpha}}-\Phi_{*}^{u_{\alpha}}\right)\right\rangle\right| \\
& \quad \leq c \alpha^{5}\left|\eta_{2}\right|^{2}\left\|\left(H_{f}+P_{f}^{2}\right)^{-\frac{1}{2}} A^{-} \Phi_{*}^{2}\right\|^{2}+\left|\kappa_{1}\right|^{2}\left\|\Phi_{\sharp}^{u_{\alpha}}-\Phi_{*}^{u_{\alpha}}\right\|_{*}^{2}=\mathcal{O}\left(\alpha^{5}\right) .
\end{aligned}
$$

Moreover,

$$
\begin{aligned}
& 4 \alpha^{\frac{3}{2}} \operatorname{Re} \eta_{2} \overline{\kappa_{1}}\left\langle A^{-} \Phi_{*}^{2} \cdot P u_{\alpha}, \Phi_{*}^{u_{\alpha}}\right\rangle \\
& \quad=\frac{8}{3} \alpha^{2}\left\|\nabla u_{\alpha}\right\|^{2} \operatorname{Re} \eta_{2} \overline{\kappa_{1}} \sum_{i=1}^{3}\left\langle\left(A^{-}\right)^{i} \Phi_{*}^{2},\left(H_{f}+P_{f}^{2}\right)^{-1}\left(A^{+}\right)^{i} \Omega_{f}\right\rangle \\
& \quad=\frac{2}{3} \alpha^{4} \operatorname{Re} \eta_{2} \overline{\kappa_{1}} \sum_{i=1}^{3}\left\langle\left(A^{-}\right)^{i} \Phi_{*}^{2},\left(H_{f}+P_{f}^{2}\right)^{-1}\left(A^{+}\right)^{i} \Omega_{f}\right\rangle \\
& \quad \geq \frac{2}{3} \alpha^{4} \operatorname{Re} \sum_{i=1}^{3}\left\langle\left(A^{-}\right)^{i} \Phi_{*}^{2},\left(H_{f}+P_{f}^{2}\right)^{-1}\left(A^{+}\right)^{i} \Omega_{f}\right\rangle-\left|\kappa_{1}-1\right| c \alpha^{4}
\end{aligned}
$$

where we used $\kappa_{1}=\mathcal{O}(1)$ (Lemma 6.1) and $\eta_{2}=1+\mathcal{O}(\alpha)$ (Theorem 5.2). Note that the right-hand side of (146) is well defined since $\left(H_{f}+P_{f}^{2}\right)^{-\frac{1}{2}} A^{+} \Omega_{f}$ $\in \mathfrak{F}$ and $\left(H_{f}+P_{f}^{2}\right)^{-\frac{1}{2}} A^{-} \Phi_{*}^{2} \in \mathfrak{F}$.

Collecting the estimates for the first and the second terms in the righthand side of (145), we arrive at

$$
\begin{aligned}
& -4 \alpha^{\frac{3}{2}} \operatorname{Re} \eta_{2}\left\langle A^{-} \Phi_{*}^{2} \cdot P u_{\alpha}, \pi^{1} \Psi^{\perp}\right\rangle \\
& \geq-\frac{8}{3} \alpha^{2}\left\|\nabla u_{\alpha}\right\|^{2} \operatorname{Re} \overline{\kappa_{1}}\left\langle\left(A^{-}\right)^{i} \Phi_{*}^{2},\left(H_{f}+P_{f}^{2}\right)^{-1} A^{+} \Omega_{f}\right\rangle \\
& \quad-\epsilon\left\|H_{f}^{\frac{1}{2}} \pi^{1} \Psi_{2}^{\perp}\right\|^{2}+\mathcal{O}\left(\alpha^{5}\right) .
\end{aligned}
$$

Here we used also $\eta_{2}=1+\mathcal{O}(\alpha)$.

As the next step, we return to (144) and estimate the second term on the right-hand side as

$$
\begin{aligned}
& 4 \alpha^{2} \operatorname{Re} 2 \eta_{3}\left\langle A^{-} \Phi_{*}^{3} \cdot P u_{\alpha}, \pi^{2} \Psi^{\perp}\right\rangle \\
& \quad=8 \alpha^{2} \operatorname{Re} \eta_{3}\left\langle H_{f}^{-\frac{1}{2}} A^{-} \Phi_{*}^{3} \cdot P u_{\alpha}, H_{f}^{\frac{1}{2}} \pi^{2} \Psi^{\perp}\right\rangle=\mathcal{O}\left(\alpha^{5}\right),
\end{aligned}
$$

where we used $H_{f}^{-\frac{1}{2}} A^{-} \Phi_{*}^{3} \in L^{2}$ and $\left\|\pi^{2} H_{f}^{\frac{1}{2}} \Delta_{\sharp}^{\perp}\right\|^{2}=\left\|\pi^{2} H_{f}^{\frac{1}{2}} \Psi^{\perp}\right\|^{2}=\mathcal{O}\left(\alpha^{4}\right)$ from Theorem 5.2. For the last term on the right-hand side of (144), we have

$$
\begin{aligned}
4 \alpha^{\frac{1}{2}} & \operatorname{Re}\left\langle A^{-} \Delta_{*}^{u_{\alpha}} \cdot \nabla u_{\alpha}, \Psi^{\perp}\right\rangle \\
= & 4 \alpha^{\frac{1}{2}} \operatorname{Re}\left\langle A^{-} \Delta_{*}^{u_{\alpha}} \cdot \nabla u_{\alpha}, \pi^{0} \Psi_{1}^{\perp}\right\rangle+4 \alpha^{\frac{1}{2}} \operatorname{Re} \overline{\kappa_{1}}\left\langle A^{-} \Delta_{*}^{u_{\alpha}} \cdot \nabla u_{\alpha}, \Phi_{\sharp}^{u_{\alpha}}\right\rangle \\
& +4 \alpha^{\frac{1}{2}} \operatorname{Re}\left\langle A^{-} \Delta_{*}^{u_{\alpha}} \cdot \nabla u_{\alpha}, \pi^{1} \Psi_{2}^{\perp}\right\rangle+4 \alpha^{\frac{1}{2}} \operatorname{Re}\left\langle A^{-} \Delta_{*}^{u_{\alpha}} \cdot \nabla u_{\alpha}, \pi^{2} \Psi^{\perp}\right\rangle \\
& +4 \alpha^{\frac{1}{2}} \operatorname{Re}\left\langle A^{-} \Delta_{*}^{u_{\alpha}} \cdot \nabla u_{\alpha}, \pi^{3} \Psi^{\perp}\right\rangle+4 \alpha^{\frac{1}{2}} \operatorname{Re}\left\langle A^{-} \Delta_{*}^{u_{\alpha}} \cdot \nabla u_{\alpha}, \pi^{n \geq 4} \Psi^{\perp}\right\rangle .
\end{aligned}
$$


We write the function $\pi^{0} \Psi_{1}^{\perp}=\left(\Psi_{1}^{\perp}\right)^{s}+\left(\Psi_{1}^{\perp}\right)^{a}$ where $\left(\Psi_{1}^{\perp}\right)^{s}$ (respectively $\left(\Psi_{1}^{\perp}\right)^{a}$ ) denotes the even (respectively odd) part of $\pi^{0} \Psi_{1}^{\perp}$. Obviously, we have

$$
\begin{aligned}
& \left|\alpha^{\frac{1}{2}} \operatorname{Re}\left\langle A^{-} \Delta_{*}^{u_{\alpha}} \cdot \nabla u_{\alpha}, \pi^{0} \Psi_{1}^{\perp}\right\rangle\right| \\
& \quad \leq c \alpha\left\|H_{f}^{\frac{1}{2}} R\right\|^{2}+\epsilon \alpha^{2}\left\|\left(\Psi_{1}^{\perp}\right)^{a}\right\|^{2}=\epsilon \alpha^{2}\left\|\left(\Psi_{1}^{\perp}\right)^{a}\right\|^{2}+\mathcal{O}\left(\alpha^{5}\right) .
\end{aligned}
$$

The constant $\epsilon$ can be chosen small for large $c$.

For the second term on the right-hand side of (149), we have

$$
\begin{aligned}
& \left|\alpha^{\frac{1}{2}} \overline{\kappa_{1}}\left\langle A^{-} \Delta_{*}^{u_{\alpha}} \cdot \nabla u_{\alpha}, \Phi_{\sharp}^{u_{\alpha}}\right\rangle\right| \\
& \quad \leq \epsilon \alpha^{5} \log \alpha^{-1}\left|\kappa_{1}\right|^{2}+c \alpha\left\|H_{f}^{\frac{1}{2}} \Delta_{*}^{u_{\alpha}}\right\|^{2}=\epsilon \alpha^{5} \log \alpha^{-1}+\mathcal{O}\left(\alpha^{5}\right) .
\end{aligned}
$$

For the third term on the right-hand side of (149), we have, since $\delta=\frac{3}{32} \alpha^{2}$

$$
\begin{aligned}
& \left|4 \alpha^{\frac{1}{2}}\left\langle A^{-} \Delta_{*}^{u_{\alpha}} \cdot \nabla u_{\alpha}, \pi^{1} \Psi_{2}^{\perp}\right\rangle\right| \\
& \quad \leq \frac{\delta}{8}\left\|\pi^{1} \Psi_{2}^{\perp}\right\|^{2}+c \alpha\left\|H_{f}^{\frac{1}{2}} \Delta_{*}^{u_{\alpha}}\right\|^{2}=\frac{\delta}{8}\left\|\pi^{1} \Psi_{2}^{\perp}\right\|^{2}+\mathcal{O}\left(\alpha^{5}\right) .
\end{aligned}
$$

Similarly,

$$
\left|4 \alpha^{\frac{1}{2}}\left\langle A^{-} \Delta_{*}^{u_{\alpha}} \cdot \nabla u_{\alpha}, \pi^{n \geq 4} \Psi^{\perp}\right\rangle\right| \leq \frac{\delta}{8}\left\|\pi^{n \geq 4} \Psi_{2}^{\perp}\right\|^{2}+\mathcal{O}\left(\alpha^{5}\right) .
$$

To complete the estimate of the last term in (149), we have to estimate two terms: $-4 \operatorname{Re} \alpha^{\frac{1}{2}}\left\langle A^{-} \Delta_{*}^{u_{\alpha}} \cdot P u_{\alpha}, \pi^{2} \Psi^{\perp}\right\rangle$ and $-4 \operatorname{Re} \alpha^{\frac{1}{2}}\left\langle A^{-} \Delta_{*}^{u_{\alpha}} \cdot P u_{\alpha}, \pi^{3} \Psi^{\perp}\right\rangle$. For the first one we have

$$
\begin{aligned}
& \left|\operatorname{Re} \alpha^{\frac{1}{2}}\left\langle A^{-} \Delta_{*}^{u_{\alpha}} \cdot P u_{\alpha}, \pi^{2} \Psi^{\perp}\right\rangle\right| \leq c \alpha\left\|H_{f}^{\frac{1}{2}} \Delta_{*}^{u_{\alpha}}\right\|^{2}+\epsilon \alpha^{2}\left\|\pi^{2} \Psi_{2}^{\perp}\right\|^{2} \\
& +\epsilon \alpha^{4}\left|\kappa_{2}\right|^{2}\left\|\pi^{0} \Psi^{\perp}\right\|^{2}+\epsilon \alpha^{6} \sum_{i=1}^{3}\left|\kappa_{2, i}\right|^{2}=\epsilon \alpha^{2}\left\|\pi^{2} \Psi_{2}^{\perp}\right\|^{2}+\mathcal{O}\left(\alpha^{5}\right) .
\end{aligned}
$$

Similarly,

$$
\begin{aligned}
& \left|\operatorname{Re} \alpha^{\frac{1}{2}}\left\langle A^{-} \Delta_{*}^{u_{\alpha}} \cdot P u_{\alpha}, \pi^{3} \Psi^{\perp}\right\rangle\right| \\
& \quad \leq c \alpha\left\|H_{f}^{\frac{1}{2}} \Delta_{*}^{u_{\alpha}}\right\|^{2}+\epsilon \alpha^{2}\left\|\pi^{3} \Psi_{2}^{\perp}\right\|^{2}+\epsilon \alpha^{6} \log \alpha^{-1}\left|\kappa_{3}\right|^{2}+\mathcal{O}\left(\alpha^{5}\right) .
\end{aligned}
$$

Collecting the estimates (149)-(154) yields

$$
\begin{aligned}
& \left|4 \operatorname{Re} \alpha^{\frac{1}{2}}\left\langle A^{-} \Delta_{*}^{u_{\alpha}} \cdot P u_{\alpha}, \Psi^{\perp}\right\rangle\right| \\
& \quad \leq \frac{\delta}{8}\left\|\Psi_{2}^{\perp}\right\|^{2}+\epsilon\left\|H_{f}^{\frac{1}{2}} \Psi_{2}^{\perp}\right\|^{2}+\epsilon \alpha^{5} \log \alpha^{-1}\left(1+\alpha\left|\kappa_{3}\right|^{2}\right)+\mathcal{O}\left(\alpha^{5}\right) .
\end{aligned}
$$

Collecting (138), (142), (147), (148) and (155) concludes the proof.

\section{A.1. Concluding the Proof of Proposition 6.1}

We can now prove the estimate on $\operatorname{Re}\left\langle H g, u_{\alpha} \Psi^{u_{\alpha}}\right\rangle$ asserted in Proposition 6.1.

Using the orthogonality (39) of $u_{\alpha}$ and $\Psi^{\perp}$, yields

$$
2 \operatorname{Re}\left\langle H \Psi^{\perp}, \Psi^{u_{\alpha}}\right\rangle=-4 \operatorname{Re}\left\langle P . P_{f} u_{\alpha} \Psi^{u_{\alpha}}, \Psi^{\perp}\right\rangle-4 \sqrt{\alpha} \operatorname{Re}\left\langle P . A(0) u_{\alpha} \Psi^{u_{\alpha}}, \Psi^{\perp}\right\rangle .
$$

Together with Lemmata A.1-A.3, this concludes the proof of Proposition 6.1. 


\section{Appendix B. Proof of Proposition 6.2}

In this section, we present the proof of Proposition 6.2.

To begin with, we establish the estimate

Proposition B.1. We have

$$
\begin{aligned}
& \left\langle H \Psi^{\perp}, \Psi^{\perp}\right\rangle \\
& \geq\left\langle H \Psi_{1}^{\perp}, \Psi_{1}^{\perp}\right\rangle+\left\langle H \Psi_{2}^{\perp}, \Psi_{2}^{\perp}\right\rangle+2 \alpha\left(\left\|A^{-} \Psi^{\perp}\right\|^{2}-\left\|A^{-} \Psi_{1}^{\perp}\right\|^{2}-\left\|A^{-} \Psi_{2}^{\perp}\right\|^{2}\right) \\
& \quad-4 \operatorname{Re}\left\langle P . P_{f} \Psi_{2}^{\perp}, \Psi_{1}^{\perp}\right\rangle-4 \alpha^{\frac{1}{2}} \operatorname{Re}\left\langle P . A(0) \Psi_{2}^{\perp}, \Psi_{1}^{\perp}\right\rangle+4 \operatorname{Re}\left\langle P_{f} \cdot A(0) \Psi_{2}^{\perp}, \Psi_{1}^{\perp}\right\rangle \\
& \quad-\epsilon M\left[\Psi_{2}^{\perp}\right]-c \alpha^{6} \log \alpha^{-1}\left|\kappa_{3}\right|^{2}-c_{0} \alpha\left\|\left(h_{\alpha}+e_{0}\right)^{\frac{1}{2}} \pi^{0} \Psi_{1}^{\perp}\right\|^{2}+\mathcal{O}\left(\alpha^{5}\right) . \quad(156)
\end{aligned}
$$

Proof. Recall that

$$
H=P^{2}-\frac{\alpha}{|x|}+T(0)-2 \operatorname{Re} P .\left(P_{f}+\alpha^{\frac{1}{2}} A(0)\right),
$$

and

$$
T(0)=:\left(P_{f}+\alpha^{\frac{1}{2}} A(0)\right)^{2}:+H_{f} .
$$

Due to the orthogonality

$$
\left\langle\pi^{n} \Psi_{1}^{\perp}, \pi^{n} \Psi_{2}^{\perp}\right\rangle_{\sharp}=0, \quad n=0,1, \ldots,
$$

and (157), (158), we obtain

$$
\begin{aligned}
&\left\langle\left(H+e_{0}\right) \Psi^{\perp}, \Psi^{\perp}\right\rangle \\
&=\left\langle\left(H+e_{0}\right) \Psi_{2}^{\perp}, \Psi_{2}^{\perp}\right\rangle+\left\langle\left(H+e_{0}\right) \Psi_{1}^{\perp}, \Psi_{1}^{\perp}\right\rangle \\
&+\sum_{n=0}^{3} 2 \alpha \operatorname{Re}\left\langle A^{-} \cdot A^{-} \pi^{n+2} \Psi_{2}^{\perp}, \pi^{n} \Psi_{1}^{\perp}\right\rangle \\
&+2 \alpha \operatorname{Re}\left\langle A^{-} \cdot A^{-} \pi^{3} \Psi_{1}^{\perp}, \pi^{1} \Psi_{2}^{\perp}\right\rangle+2 \alpha\left(\left\|A^{-} \Psi^{\perp}\right\|^{2}-\left\|A^{-} \Psi_{1}^{\perp}\right\|^{2}-\left\|A^{-} \Psi_{2}^{\perp}\right\|^{2}\right) \\
&-4 \operatorname{Re}\left\langle P . P_{f} \Psi_{2}^{\perp}, \Psi_{1}^{\perp}\right\rangle-4 \alpha^{\frac{1}{2}} \operatorname{Re}\left\langle P \cdot A(0) \Psi_{2}^{\perp}, \Psi_{1}^{\perp}\right\rangle+4 \alpha^{\frac{1}{2}} \operatorname{Re}\left\langle P_{f} \cdot A(0) \Psi_{2}^{\perp}, \Psi_{1}^{\perp}\right\rangle .
\end{aligned}
$$

We have

$$
\begin{aligned}
2 \alpha \operatorname{Re}\left\langle A^{-} \cdot A^{-} \pi^{5} \Psi_{2}^{\perp}, \pi^{3} \Psi_{1}^{\perp}\right\rangle & \geq-\epsilon\left\|H_{f}^{\frac{1}{2}} \pi^{5} \Psi_{2}^{\perp}\right\|^{2}-c \alpha^{2}\left\|\pi^{3} \Psi_{1}^{\perp}\right\|^{2} \\
& \geq-\epsilon\left\|H_{f}^{\frac{1}{2}} \pi^{5} \Psi_{2}^{\perp}\right\|^{2}-c \alpha^{7} \log \alpha^{-1}\left|\kappa_{3}\right|^{2} .
\end{aligned}
$$

Similarly,

$$
\begin{aligned}
& 2 \alpha \operatorname{Re}\left\langle A^{-} . A^{-} \pi^{4} \Psi_{2}^{\perp}, \pi^{2} \Psi_{1}^{\perp}\right\rangle \\
& \quad \geq-\epsilon\left\|H_{f}^{\frac{1}{2}} \pi^{4} \Psi_{2}^{\perp}\right\|^{2}-c \alpha^{4}\left|\kappa_{2}\right|^{2}\left\|\pi^{0} \Psi_{1}^{\perp}\right\|^{2}-\sum_{i=1}^{3} c \alpha^{6}\left|\kappa_{2, i}\right|^{2} \\
& \quad \geq-\epsilon\left\|H_{f}^{\frac{1}{2}} \pi^{4} \Psi_{2}^{\perp}\right\|^{2}+\mathcal{O}\left(\alpha^{5}\right) .
\end{aligned}
$$

To estimate the term $2 \alpha \operatorname{Re}\left\langle A^{-} . A^{-} \pi^{3} \Psi_{2}^{\perp}, \pi^{1} \Psi_{1}^{\perp}\right\rangle$ we rewrite it as $2 \alpha \operatorname{Re}$ $\left\langle\pi^{3} \Psi_{2}^{\perp}, A^{+} . A^{+} \kappa_{1} \Phi_{\sharp}^{u_{\alpha}}\right\rangle$ and use that $\left\langle\pi^{3} \Psi_{2}^{\perp},\left(H_{f}+P_{f}^{2}\right)^{-1} A^{+} \cdot A^{+} \Phi_{\sharp}^{u_{\alpha}}\right\rangle_{\sharp}=0$. 
This yields, using Lemma C.6

$$
\begin{aligned}
& \alpha \operatorname{Re}\left\langle A^{-} . A^{-} \pi^{3} \Psi_{2}^{\perp}, \pi^{1} \Psi_{1}^{\perp}\right\rangle \\
& \quad=-\alpha \operatorname{Re}\left\langle\pi^{3} \Psi_{2}^{\perp},\left(h_{\alpha}+e_{0}\right)\left(H_{f}+P_{f}^{2}\right)^{-1} A^{+} \cdot A^{+} \kappa_{1} \Phi_{\sharp}^{u_{\alpha}}\right\rangle \\
& \quad \geq-\epsilon \alpha^{2}\left\|\pi^{3} \Psi_{2}^{\perp}\right\|^{2}+c \alpha^{7} \log \alpha^{-1} .
\end{aligned}
$$

Similarly, if $\left\|\pi^{0} \Psi_{1}^{\perp}\right\|>\alpha^{\frac{3}{2}}$,

$$
\begin{aligned}
2 \alpha \operatorname{Re} & \left\langle A^{-} . A^{-} \pi^{2} \Psi_{2}^{\perp}, \pi^{0} \Psi_{1}^{\perp}\right\rangle \\
= & -2 \alpha \operatorname{Re}\left\langle\pi^{2} \Psi_{2}^{\perp},\left(h_{\alpha}+e_{0}\right)\left(H_{f}+P_{f}^{2}\right)^{-1} A^{+} \cdot A^{+} \pi^{0} \Psi_{1}^{\perp}\right\rangle \\
\geq & -c \alpha\left\|\left(h_{\alpha}+e_{0}\right)^{\frac{1}{2}} \pi^{2} \Psi_{2}^{\perp}\right\|^{2}-c \alpha\left\|\left(h_{\alpha}+e_{0}\right)^{\frac{1}{2}}\left(H_{f}+P_{f}^{2}\right)^{-1} A^{+} . A^{+} \pi^{0} \Psi_{1}^{\perp}\right\|^{2} \\
\geq & -c \alpha\left\|P \pi^{2} \Psi_{2}^{\perp}\right\|^{2}+c \alpha\left\||x|^{-\frac{1}{2}} \pi^{2} \Psi_{2}^{\perp}\right\|^{2} \\
& -c \alpha e_{0}\left\|\pi^{2} \Psi_{2}^{\perp}\right\|^{2}-c_{0} \alpha\left\|\left(h_{\alpha}+e_{0}\right)^{\frac{1}{2}} \pi^{0} \Psi_{1}^{\perp}\right\|^{2} \\
\geq & -c \alpha\left\|P \pi^{2} \Psi_{2}^{\perp}\right\|^{2}-\epsilon \alpha^{2}\left\|\pi^{2} \Psi_{2}^{\perp}\right\|^{2}-c_{0} \alpha\left\|\left(h_{\alpha}+e_{0}\right)^{\frac{1}{2}} \pi^{0} \Psi_{1}^{\perp}\right\|^{2} .
\end{aligned}
$$

If $\left\|\pi^{0} \Psi_{1}^{\perp}\right\| \leq \alpha^{\frac{3}{2}}$, we have instead

$$
\begin{aligned}
2 \alpha \operatorname{Re}\left\langle A^{-} . A^{-} \pi^{2} \Psi_{2}^{\perp}, \pi^{0} \Psi_{1}^{\perp}\right\rangle & \geq-\epsilon\left\|H_{f}^{\frac{1}{2}} \pi^{2} \Psi_{2}^{\perp}\right\|^{2}-c \alpha^{2}\left\|\pi^{0} \Psi_{1}^{\perp}\right\|^{2} \\
& \geq-\epsilon\left\|H_{f}^{\frac{1}{2}} \pi^{2} \Psi_{2}^{\perp}\right\|^{2}+\mathcal{O}\left(\alpha^{5}\right) .
\end{aligned}
$$

Finally, using Lemma C.7 yields

$$
2 \alpha \operatorname{Re}\left\langle A^{-} . A^{-} \pi^{3} \Psi_{1}^{\perp}, \pi^{1} \Psi_{2}^{\perp}\right\rangle \geq-\epsilon\left\|H_{f}^{\frac{1}{2}} \Psi_{2}^{\perp}\right\|^{2}+\mathcal{O}\left(\alpha^{5}\right) .
$$

Collecting (159)-(164) concludes the proof of the proposition.

In the rest of this section, we estimate further terms in (156).

\section{B.1. Estimate of Crossed Terms Involving $\Psi_{1}^{\perp}$ and $\Psi_{2}^{\perp}$}

\section{Lemma B.1.}

$$
2 \alpha\left(\left\|A^{-} \Psi^{\perp}\right\|^{2}-\left\|A^{-} \Psi_{1}^{\perp}\right\|^{2}-\left\|A^{-} \Psi_{2}^{\perp}\right\|^{2}\right) \geq-\epsilon M\left[\Psi_{2}^{\perp}\right]+\mathcal{O}\left(\alpha^{5}\right) .
$$

Proof. Obviously, the left-hand side of (165) is equal to

$$
\begin{aligned}
4 \alpha \operatorname{Re} & \left\langle A^{-} \Psi_{1}^{\perp}, A^{-} \Psi_{2}^{\perp}\right\rangle \geq-c \alpha \sum_{n}\left\|H_{f}^{\frac{1}{2}} \pi^{n} \Psi_{1}^{\perp}\right\|\left\|H_{f}^{\frac{1}{2}} \pi^{n} \Psi_{2}^{\perp}\right\| \\
\geq & -\epsilon\left\|H_{f}^{\frac{1}{2}} \pi^{1} \Psi_{2}^{\perp}\right\|^{2}-c \alpha^{2}\left|\kappa_{1}\right|^{2}\left\|H_{f}^{\frac{1}{2}} \Phi_{\sharp}^{u_{\alpha}}\right\|^{2} \\
& -\sum_{n \neq 1} c \alpha\left(3\left\|H_{f}^{\frac{1}{2}} \pi^{n} \Psi_{2}^{\perp}\right\|^{2}+2\left\|H_{f}^{\frac{1}{2}} \pi^{n} \Psi^{\perp}\right\|^{2}\right) \\
\geq & -\epsilon\left\|H_{f}^{\frac{1}{2}} \Psi_{2}^{\perp}\right\|^{2}+\mathcal{O}\left(\alpha^{5}\right),
\end{aligned}
$$

where in the last inequality we used (210) of Lemma C.4, and (45) of Theorem 5.2.

\section{Lemma B.2.}

$$
\left|\left\langle P . P_{f} \Psi_{2}^{\perp}, \Psi_{1}^{\perp}\right\rangle\right| \leq \epsilon M\left[\Psi_{2}^{\perp}\right]+c \alpha^{7} \log \alpha^{-1}\left|\kappa_{3}\right|^{2}+\mathcal{O}\left(\alpha^{5}\right)
$$


Proof. We have

$$
\begin{aligned}
& \left\langle P . P_{f} \Psi_{2}^{\perp}, \Psi_{1}^{\perp}\right\rangle \\
& \quad=\left\langle P_{f} \pi^{1} \Psi_{2}^{\perp}, P \pi^{1} \Psi_{1}^{\perp}\right\rangle+\left\langle P_{f} \pi^{2} \Psi_{2}^{\perp}, P \pi^{2} \Psi_{1}^{\perp}\right\rangle+\left\langle P_{f} \pi^{3} \Psi_{2}^{\perp}, P \pi^{3} \Psi_{1}^{\perp}\right\rangle .
\end{aligned}
$$

Obviously, using Lemma C.4 and the equality $\kappa_{1}=\mathcal{O}(1)$ from Lemma 6.1, yields

$$
\begin{aligned}
& \left\langle P_{f} \pi^{1} \Psi_{2}^{\perp}, P \pi^{1} \Psi_{1}^{\perp}\right\rangle \mid \\
& \quad \leq \epsilon\left\|H_{f}^{\frac{1}{2}} \Psi_{2}^{\perp}\right\|^{2}+\left|\kappa_{1}\right|^{2}\left\|P\left|P_{f}\right|^{\frac{1}{2}} \Phi_{\sharp}^{u_{\alpha}}\right\|^{2} \leq \epsilon\left\|H_{f}^{\frac{1}{2}} \Psi_{2}^{\perp}\right\|^{2}+\mathcal{O}\left(\alpha^{5}\right) .
\end{aligned}
$$

We also have, by definition of $\pi^{2} \Psi_{1}^{\perp}$ and using the estimates $\kappa_{2, i}=\mathcal{O}(1)$ from Lemma 6.1,

$$
\left.\left\langle P_{f} \pi^{2} \Psi_{2}^{\perp}, P \pi^{2} \Psi_{1}^{\perp}\right\rangle\left|\leq \epsilon\left\|H_{f}^{\frac{1}{2}} \Psi_{2}^{\perp}\right\|^{2}+c\right| \kappa_{2}\right|^{2} \alpha^{2}\left\|P \pi^{0} \Psi_{1}^{\perp}\right\|^{2}+\mathcal{O}\left(\alpha^{6}\right) .
$$

We next bound the second term on the right-hand side of (169). Notice that by definition of $\kappa_{2}$, this term is nonzero only if $\left\|\pi^{0} \Psi_{1}^{\perp}\right\|^{2}>\alpha^{3}$, which implies, with Lemma 6.1, that $\left\|P \pi^{0} \Psi_{1}^{\perp}\right\|^{2} \leq c \alpha\left\|\pi^{0} \Psi_{1}^{\perp}\right\|^{2}$. The inequality (169) can thus be rewritten as

$$
\begin{aligned}
& \left|\left\langle P . P_{f} \pi^{2} \Psi_{2}^{\perp}, \pi^{2} \Psi_{1}^{\perp}\right\rangle\right| \\
& \quad \leq \epsilon\left\|H_{f}^{\frac{1}{2}} \Psi_{2}^{\perp}\right\|^{2}+c\left|\kappa_{2}\right|^{2} \alpha^{3}\left\|\pi^{0} \Psi_{1}^{\perp}\right\|^{2}+\mathcal{O}\left(\alpha^{6}\right) \leq \epsilon\left\|H_{f}^{\frac{1}{2}} \Psi_{2}^{\perp}\right\|^{2}+\mathcal{O}\left(\alpha^{5}\right),
\end{aligned}
$$

using in the last inequality that $\left|\kappa_{2}\right|\left\|\pi^{0} \Psi_{1}^{\perp}\right\|=\mathcal{O}(\alpha)$ (see Lemma 6.1).

For the second term on the right-hand side of (167), using (212) from Lemma C.4 yields

$$
\left\langle\pi^{3} P_{f} \Psi_{2}^{\perp}, \pi^{3} P \Psi_{1}^{\perp}\right\rangle \leq \epsilon\left\|\pi^{3} H_{f}^{\frac{1}{2}} \Psi_{2}^{\perp}\right\|^{2}+c \alpha^{7} \log \alpha^{-1}\left|\kappa_{3}\right|^{2} .
$$

The inequalities (167), (170) and (171) prove the lemma.

\section{Lemma B.3.}

$$
-4 \alpha^{\frac{1}{2}} \operatorname{Re}\left\langle P \cdot A^{+} \Psi_{2}^{\perp}, \Psi_{1}^{\perp}\right\rangle \geq-\epsilon M\left[\Psi_{2}^{\perp}\right]+\mathcal{O}\left(\alpha^{5}\right) .
$$

Proof. Since $\pi^{n>3} \Psi_{1}^{\perp}=0, \pi^{0} \Psi_{2}^{\perp}=0$ and

$$
\left\|H_{f}^{\frac{1}{2}} \pi^{n \neq 1} \Psi_{1}^{\perp}\right\|^{2} \leq 2\left\|H_{f}^{\frac{1}{2}} \pi^{n \neq 1} \Psi^{\perp}\right\|^{2}+2\left\|H_{f}^{\frac{1}{2}} \pi^{n \neq 1} \Psi_{2}^{\perp}\right\|^{2} \leq c M\left[\Psi_{2}^{\perp}\right]+\mathcal{O}\left(\alpha^{4}\right),
$$

(see (45) of Theorem 5.2) we obtain

$$
\begin{aligned}
& 4 \alpha^{\frac{1}{2}} \operatorname{Re}\left\langle P . A^{+} \Psi_{2}^{\perp}, \Psi_{1}^{\perp}\right\rangle \\
& \quad \leq \epsilon\left\|\pi^{n \leq 2} P \Psi_{2}^{\perp}\right\|^{2}+c \alpha\left\|\pi^{n \geq 2} H_{f}^{\frac{1}{2}} \Psi_{1}^{\perp}\right\|^{2} \leq \epsilon M\left[\Psi_{2}^{\perp}\right]+\mathcal{O}\left(\alpha^{5}\right) .
\end{aligned}
$$

\section{Lemma B.4.}

$$
-4 \alpha^{\frac{1}{2}} \operatorname{Re}\left\langle P . A^{-} \Psi_{2}^{\perp}, \Psi_{1}^{\perp}\right\rangle \geq-\epsilon M\left[\Psi_{2}^{\perp}\right]+\mathcal{O}\left(\alpha^{5}\right) .
$$


Proof.

$$
\begin{aligned}
& -4 \alpha^{\frac{1}{2}} \operatorname{Re}\left\langle P . A^{-} \Psi_{2}^{\perp}, \Psi_{1}^{\perp}\right\rangle \geq-\epsilon\left\|H_{f}^{\frac{1}{2}} \Psi_{2}^{\perp}\right\|^{2}-c \alpha\left\|P \Psi_{1}^{\perp}\right\|^{2} \\
& \geq-\epsilon\left\|H_{f}^{\frac{1}{2}} \Psi_{2}^{\perp}\right\|^{2}-c \alpha\left(\left\|\pi^{n=0,2,3,4} P \Psi^{\perp}\right\|^{2}+\left\|\pi^{n=0,2,3,4} P \Psi_{2}^{\perp}\right\|^{2}\right) \\
& -c \alpha\left|\kappa_{1}\right|^{2}\left\|P \Phi_{\sharp}^{u_{\alpha}}\right\|^{2} \\
& \geq-\epsilon\left\|H_{f}^{\frac{1}{2}} \Psi_{2}^{\perp}\right\|^{2}-\epsilon\left\|\pi^{n \leq 4} P \Psi_{2}^{\perp}\right\|^{2}+\mathcal{O}\left(\alpha^{6} \log \alpha^{-1}\right) \\
& \geq-\epsilon M\left[\Psi_{2}^{\perp}\right]+\mathcal{O}\left(\alpha^{6} \log \alpha^{-1}\right),
\end{aligned}
$$

using (212) of Lemma C.4.

\section{Lemma B.5.}

$4 \alpha^{\frac{1}{2}} \operatorname{Re}\left\langle P_{f} \cdot A(0) \Psi_{2}^{\perp}, \Psi_{1}^{\perp}\right\rangle \geq 4 \alpha^{\frac{1}{2}} \operatorname{Re}\left\langle\pi^{2} \Psi_{2}^{\perp}, P_{f} \cdot A^{+} \Phi_{*}^{u_{\alpha}}\right\rangle-\epsilon M\left[\Psi_{2}^{\perp}\right]+\mathcal{O}\left(\alpha^{5}\right)$.

Proof. We have

$$
\begin{aligned}
& 4 \alpha^{\frac{1}{2}} \operatorname{Re}\left\langle P_{f} \cdot A^{-} \Psi_{2}^{\perp}, \Psi_{1}^{\perp}\right\rangle \\
& \quad \geq-\epsilon\left\|H_{f}^{\frac{1}{2}} \Psi_{2}^{\perp}\right\|^{2}-c \alpha\left\|\pi^{n \geq 2} P_{f} \Psi_{1}^{\perp}\right\|^{2}+4 \alpha^{\frac{1}{2}} \operatorname{Re}\left\langle P_{f} \cdot A^{-} \pi^{2} \Psi_{2}^{\perp}, \kappa_{1} \Phi_{\sharp}^{u_{\alpha}}\right\rangle \\
& \geq-\epsilon\left\|H_{f}^{\frac{1}{2}} \Psi_{2}^{\perp}\right\|^{2}-c \alpha\left\|\pi^{n=2,3} H_{f}^{\frac{1}{2}} \Psi^{\perp}\right\|^{2}+4 \alpha^{\frac{1}{2}} \operatorname{Re}\left\langle P_{f} \cdot A^{-} \pi^{2} \Psi_{2}^{\perp}, \kappa_{1} \Phi_{\sharp}^{u_{\alpha}}\right\rangle \\
& \geq-\epsilon M\left[\Psi_{2}^{\perp}\right]+4 \alpha^{\frac{1}{2}} \operatorname{Re}\left\langle P_{f} \cdot A^{-} \pi^{2} \Psi_{2}^{\perp}, \kappa_{1} \Phi_{\sharp}^{u_{\alpha}}\right\rangle+\mathcal{O}\left(\alpha^{5}\right) .
\end{aligned}
$$

We estimate the second term on the right-hand side as follows:

$$
\begin{aligned}
& 4 \alpha^{\frac{1}{2}} \operatorname{Re}\left\langle P_{f} \cdot A^{-} \pi^{2} \Psi_{2}^{\perp}, \kappa_{1} \Phi_{\sharp}^{u_{\alpha}}\right\rangle \\
& \quad \geq 4 \alpha^{\frac{1}{2}} \operatorname{Re}\left\langle P_{f} \cdot A^{-} \pi^{2} \Psi_{2}^{\perp}, \Phi_{\sharp}^{u_{\alpha}}\right\rangle-\epsilon\left\|H_{f}^{\frac{1}{2}} \pi^{2} \Psi_{2}^{\perp}\right\|^{2}-c \alpha|\kappa-1|^{2}\left\|P_{f} \Phi_{\sharp}^{u_{\alpha}}\right\|^{2} \\
& \quad \geq 4 \alpha^{\frac{1}{2}} \operatorname{Re}\left\langle P_{f} \cdot A^{-} \pi^{2} \Psi_{2}^{\perp}, \Phi_{*}^{u_{\alpha}}\right\rangle-\epsilon\left\|H_{f}^{\frac{1}{2}} \pi^{2} \Psi_{2}^{\perp}\right\|^{2}+\mathcal{O}\left(\alpha^{5}\right),
\end{aligned}
$$

where we used $\left|\kappa_{1}-1\right|^{2}=\mathcal{O}(\alpha)$ from Lemma 6.1, $\left\|\Phi_{\sharp}^{u_{\alpha}}\right\|_{*}^{2}=\mathcal{O}\left(\alpha^{3}\right)$ from Lemma C.4, and $\left\|P_{f}\left(\Phi_{\sharp}^{u_{\alpha}}-\Phi_{*}^{u_{\alpha}}\right)\right\|^{2}=\mathcal{O}\left(\alpha^{4}\right)$ from Lemma C.5.

We also have, using $P_{f} \cdot A^{+}=A^{+} . P_{f}$,

$$
\begin{gathered}
4 \alpha^{\frac{1}{2}} \operatorname{Re}\left\langle P_{f} \cdot A^{+} \Psi_{2}^{\perp}, \Psi_{1}^{\perp}\right\rangle=4 \alpha^{\frac{1}{2}} \operatorname{Re}\left\langle\pi^{n \leq 2} P_{f} \Psi_{2}^{\perp}, A^{-} \pi^{n \geq 2} \Psi_{1}^{\perp}\right\rangle \\
\geq-\epsilon\left\|H_{f}^{\frac{1}{2}} \Psi_{2}^{\perp}\right\|^{2}-c \alpha\left\|H_{f}^{\frac{1}{2}} \pi^{n \geq 2} \Psi_{1}^{\perp}\right\|^{2} \geq-\epsilon M\left[\Psi_{2}^{\perp}\right]+\mathcal{O}\left(\alpha^{5}\right),
\end{gathered}
$$

where $\left\|H_{f}^{\frac{1}{2}} \pi^{n \geq 2} \Psi_{1}^{\perp}\right\|$ has been estimated as $\left\|P \Psi_{1}^{\perp}\right\|$ in the proof of Lemma B.4.

\section{B.2. Estimates of the Term $\left\langle\left(H+e_{0}\right) \Psi_{1}^{\perp}, \Psi_{1}^{\perp}\right\rangle$}

Due to (157) and (158), one finds

$$
\begin{aligned}
& \left\langle\left(H+e_{0}\right) \Psi_{1}^{\perp}, \Psi_{1}^{\perp}\right\rangle=\left\langle\Psi_{1}^{\perp}, \Psi_{1}^{\perp}\right\rangle_{\sharp}-2 \operatorname{Re}\left\langle P .\left(P_{f}+\alpha^{\frac{1}{2}} A(0)\right) \Psi_{1}^{\perp}, \Psi_{1}^{\perp}\right\rangle \\
& \quad+2 \alpha^{\frac{1}{2}} \operatorname{Re}\left\langle P_{f} \cdot A(0) \Psi_{1}^{\perp}, \Psi_{1}^{\perp}\right\rangle+2 \alpha\left\|A^{-} \Psi_{1}^{\perp}\right\|^{2}+2 \alpha \operatorname{Re}\left\langle A^{-} \cdot A^{-} \Psi_{1}^{\perp}, \Psi_{1}^{\perp}\right\rangle
\end{aligned}
$$

We estimate the terms in (172) below. 
Lemma B.6. We have

$$
\begin{aligned}
& -2 \operatorname{Re}\left\langle P .\left(P_{f}+\alpha^{\frac{1}{2}} A(0)\right) \Psi_{1}^{\perp}, \Psi_{1}^{\perp}\right\rangle \\
& \quad \geq-4 \alpha^{\frac{1}{2}} \operatorname{Re}\left\langle A^{-} \Phi_{\sharp}^{u_{\alpha}}, P \pi^{0}\left(\Psi_{1}^{\perp}\right)^{s}\right\rangle-\epsilon M\left[\Psi_{2}^{\perp}\right]-\left|\kappa_{1}-1\right| c \alpha^{4}+\mathcal{O}\left(\alpha^{5}\right),
\end{aligned}
$$

where $\pi^{0}\left(\Psi_{1}^{\perp}\right)^{s}=\pi^{0} \Psi_{1}^{\perp}-\pi^{0}\left(\Psi_{1}^{\perp}\right)^{a}$ is the even part of $\pi^{0} \Psi_{1}^{\perp}$.

Proof. Using $\left\langle\Phi_{*}^{2}, P_{f}^{i} \Phi_{*}^{2}\right\rangle=0$ (see Lemma C.2), the symmetry of $u_{\alpha}$, and $\left\langle P . P_{f} \Phi_{\sharp}^{u_{\alpha}}, \Phi_{\sharp}^{u_{\alpha}}\right\rangle=0$, we obtain

$$
\begin{aligned}
\left|2\left\langle P . P_{f} \Psi_{1}^{\perp}, \Psi_{1}^{\perp}\right\rangle\right| & =\left|2\left\langle P_{f} \alpha \kappa_{2} \Phi_{*}^{2} \pi^{0} \Psi_{1}^{\perp}, P \alpha \sum_{i=1}^{3} \kappa_{2, i}\left(H_{f}+P_{f}^{2}\right)^{-1} W_{i} \frac{\partial u_{\alpha}}{\partial x_{i}}\right\rangle\right| \\
& \leq c \alpha^{3}\left|\kappa_{2}\right|^{2}\left\|\pi^{0} \Psi_{1}^{\perp}\right\|^{2}+c \alpha^{5} \sum_{i=1}^{3}\left|\kappa_{2, i}\right|^{2}=\mathcal{O}\left(\alpha^{5}\right),
\end{aligned}
$$

where in the last inequality we used Lemma 6.1.

We also have, using again the symmetry of $u_{\alpha}$,

$$
\begin{aligned}
&-2 \alpha^{\frac{1}{2}} \operatorname{Re}\left\langle P \cdot A(0) \Psi_{1}^{\perp}, \Psi_{1}^{\perp}\right\rangle=-4 \alpha^{\frac{1}{2}} \operatorname{Re}\left\langle P \cdot A^{-} \Psi_{1}^{\perp}, \Psi_{1}^{\perp}\right\rangle \\
&=-4 \alpha^{\frac{1}{2}} \operatorname{Re}\left\langle A^{-} \kappa_{1} \Phi_{\sharp}^{u_{\alpha}}, P \pi^{0}\left(\Psi_{1}^{\perp}\right)^{s}\right\rangle-4 \alpha^{\frac{1}{2}} \operatorname{Re}\left\langle A^{-} \kappa_{2} \alpha \Phi_{*}^{2} \pi^{0} \Psi_{1}^{\perp}, P \kappa_{1} \Phi_{\sharp}^{u_{\alpha}}\right\rangle \\
&-4 \alpha^{\frac{1}{2}} \operatorname{Re}\left\langle A^{-} \pi^{3} \Psi_{1}^{\perp}, P \alpha \kappa_{2} \Phi_{*}^{2} \pi^{0} \Psi_{1}^{\perp}\right\rangle \\
& \geq-4 \alpha^{\frac{1}{2}} \operatorname{Re}\left\langle A^{-} \kappa_{1} \Phi_{\sharp}^{u_{\alpha}}, P \pi^{0}\left(\Psi_{1}^{\perp}\right)^{s}\right\rangle-c \alpha\left\|H_{f}^{\frac{1}{2}} \pi^{3} \Psi_{1}^{\perp}\right\|^{2}-c \alpha^{2}\left\|P \pi^{0} \Psi_{1}^{\perp}\right\|^{2}\left|\kappa_{2}\right|^{2} \\
&-4 \alpha^{\frac{1}{2}} \operatorname{Re}\left\langle|k|^{-\frac{1}{6}} A^{-} \kappa_{2} \alpha \Phi_{*}^{2} \pi^{0} \Psi_{1}^{\perp},|k|^{\frac{1}{6}} \kappa_{1} P \Phi_{\sharp}^{u_{\alpha}}\right\rangle \\
& \geq-4 \alpha^{\frac{1}{2}} \operatorname{Re}\left\langle A^{-} \kappa_{1} \Phi_{\sharp}^{u_{\alpha}}, P \pi^{0}\left(\Psi_{1}^{\perp}\right)^{s}\right\rangle-c \alpha\left\|H_{f}^{\frac{1}{2}} \pi^{3} \Psi^{\perp}\right\|^{2}-c \alpha\left\|H_{f}^{\frac{1}{2}} \pi^{3} \Psi_{2}^{\perp}\right\|^{2} \\
&-c \alpha^{5}-c \alpha^{\frac{3}{2}}\left|\kappa_{2}\right|\left\|\pi^{0} \Psi_{1}^{\perp}\right\|\left\||k|^{\frac{1}{6}} P \Phi_{\sharp}^{u_{\alpha}}\right\| \\
& \geq-4 \alpha^{\frac{1}{2}} \operatorname{Re}\left\langle A^{-} \kappa_{1} \Phi_{\sharp}^{u_{\alpha}}, P \pi^{0}\left(\Psi_{1}^{\perp}\right)^{s}\right\rangle-\epsilon M\left[\Psi_{2}^{\perp}\right]-c \alpha^{5},
\end{aligned}
$$

where we used Theorem 5.2 and Lemma C.4.

Moreover, because $\left\|A^{-} \Phi_{*}^{u_{\alpha}}\right\|=\mathcal{O}\left(\alpha^{\frac{3}{2}}\right)$ and $\left\|P \pi^{0} \Psi_{1}^{\perp}\right\|=\mathcal{O}\left(\alpha^{2}\right)$, we obtain

$$
\begin{aligned}
& -4 \operatorname{Re} \alpha^{\frac{1}{2}} \operatorname{Re}\left\langle A^{-} \kappa_{1} \Phi_{\sharp}^{u_{\alpha}}, P \pi^{0}\left(\Psi_{1}^{\perp}\right)^{s}\right\rangle \\
& \quad \geq-4 \alpha^{\frac{1}{2}} \operatorname{Re}\left\langle A^{-} \Phi_{\sharp}^{u_{\alpha}}, P \pi^{0}\left(\Psi_{1}^{\perp}\right)^{s}\right\rangle-\left|\kappa_{1}-1\right| c \alpha^{4} .
\end{aligned}
$$

This estimate, together with (173) and (174), proves the lemma.

Lemma B.7. We have

$$
\begin{aligned}
& 2 \alpha^{\frac{1}{2}} \operatorname{Re}\left\langle P_{f} \cdot A(0) \Psi_{1}^{\perp}, \Psi_{1}^{\perp}\right\rangle \\
& \geq \geq \frac{2}{3} \alpha^{4} \operatorname{Re} \sum_{i} \kappa_{2, i}\left\langle P_{f} \cdot A^{-}\left(H_{f}+P_{f}^{2}\right)^{-1} W_{i},\left(H_{f}+P_{f}^{2}\right)^{-1}\left(A^{+}\right)^{i} \Omega_{f}\right\rangle \\
& \quad-\left|\kappa_{1}-1\right| c \alpha^{4}+\mathcal{O}\left(\alpha^{5}\right) .
\end{aligned}
$$


Proof. The following holds:

$$
\begin{aligned}
& 2 \alpha^{\frac{1}{2}} \operatorname{Re}\left\langle P_{f} \cdot A(0) \Psi_{1}^{\perp}, \Psi_{1}^{\perp}\right\rangle \\
& =4 \alpha^{\frac{1}{2}} \operatorname{Re}\left\langle P_{f} \cdot A^{-} \pi^{3} \Psi_{1}^{\perp}, \pi^{2} \Psi_{1}^{\perp}\right\rangle+4 \alpha \operatorname{Re}\left\langle P_{f} \cdot A^{-} \pi^{2} \Psi_{1}^{\perp}, \kappa_{1} \Phi_{\sharp}^{u_{\alpha}}\right\rangle \\
& =4 \alpha^{\frac{3}{2}} \operatorname{Re} \sum_{i=1}^{3} \overline{\kappa_{2, i}}\left\langle\left|k_{1}\right|^{\frac{1}{6}}\left|k_{2}\right|^{\frac{1}{6}} A^{-} \pi^{3} \Psi_{1}^{\perp}, P_{f}\left|k_{1}\right|^{-\frac{1}{6}}\left|k_{2}\right|^{-\frac{1}{6}}\left(H_{f}+P_{f}^{2}\right)^{-1} W_{i} \frac{\partial u_{\alpha}}{\partial x_{i}}\right\rangle \\
& \quad+4 \alpha^{\frac{3}{2}} \operatorname{Re} \kappa_{2}\left\langle\left|k_{1}\right|^{\frac{1}{6}}\left|k_{2}\right|^{\frac{1}{6}} A^{-} \pi^{3} \Psi_{1}^{\perp},\left|k_{1}\right|^{-\frac{1}{6}}\left|k_{2}\right|^{-\frac{1}{6}} P_{f} \Phi_{*}^{2} \pi^{0} \Psi_{1}^{\perp}\right\rangle \\
& \quad+4 \alpha^{\frac{1}{2}} \operatorname{Re}\left\langle P_{f} \cdot A^{-} \pi^{2} \Psi_{1}^{\perp}, \kappa_{1} \Phi_{\sharp}^{u_{\alpha}}\right\rangle .
\end{aligned}
$$

Applying the Schwarz inequality and the estimates $\|\left|k_{1}\right|^{\frac{1}{6}}\left|k_{2}\right|^{\frac{1}{6}} A^{-} \pi^{3}$ $\Psi_{1}^{\perp} \|^{2}=\mathcal{O}\left(\alpha^{5}\right)\left(\right.$ Lemma C.6), $\kappa_{2, i}=\mathcal{O}(1)$ (Lemma 6.1), and $\left\|\nabla u_{\alpha}\right\|^{2}=\mathcal{O}\left(\alpha^{2}\right)$, we see that the first term on the right-hand side of (176) is $\mathcal{O}\left(\alpha^{5}\right)$. Applying also the estimate $\left|\kappa_{2}\right|\left\|\pi^{0} \Psi_{1}^{\perp}\right\|=\mathcal{O}(\alpha)$ (Lemma 6.1), we obtain that the second term on the right-hand side of (176) is also $\mathcal{O}\left(\alpha^{5}\right)$.

Finally, we estimate $4 \alpha^{\frac{1}{2}} \operatorname{Re}\left\langle P_{f} \cdot A^{-} \pi^{2} \Psi_{1}^{\perp}, \kappa_{1} \Phi_{\sharp}^{u_{\alpha}}\right\rangle$. The following inequality holds:

$$
4 \alpha^{\frac{1}{2}} \operatorname{Re}\left\langle P_{f} \cdot A^{-} \pi^{2} \Psi_{1}^{\perp}, \kappa_{1} \Phi_{\sharp}^{u_{\alpha}}\right\rangle\left|\geq 4 \alpha^{\frac{1}{2}} \operatorname{Re}\left\langle P_{f} \cdot A^{-} \pi^{2} \Psi_{1}^{\perp}, \Phi_{\sharp}^{u_{\alpha}}\right\rangle-\right| \kappa_{1}-1 \mid c \alpha^{4},
$$

whose proof is similar to the one of (175). Next we get

$$
\begin{gathered}
\left|4 \alpha^{\frac{1}{2}} \operatorname{Re}\left\langle P_{f} \cdot A^{-} \pi^{2} \Psi_{1}^{\perp}, \Phi_{\sharp}^{u_{\alpha}}\right\rangle-4 \alpha^{\frac{1}{2}} \operatorname{Re}\left\langle P_{f} \cdot A^{-} \pi^{2} \Psi_{1}^{\perp}, \Phi_{*}^{u_{\alpha}}\right\rangle\right| \\
\leq \alpha^{\frac{1}{2}}\left\|H_{f}^{\frac{1}{2}} \pi^{2} \Psi_{1}^{\perp}\right\|\left\|P_{f}\left(\Phi_{\sharp}^{u_{\alpha}}-\Phi_{*}^{u_{\alpha}}\right)\right\|=\mathcal{O}\left(\alpha^{6}|\log \alpha|^{\frac{1}{2}}\right),
\end{gathered}
$$

using $\left\|P_{f}\left(\Phi_{\sharp}^{u_{\alpha}}-\Phi_{*}^{u_{\alpha}}\right)\right\|=\mathcal{O}\left(\alpha^{\frac{7}{2}}|\log \alpha|^{\frac{1}{2}}\right)$ (see Lemma C.5) and $\left\|H_{f}^{\frac{1}{2}} \pi^{2} \Psi_{1}^{\perp}\right\|=$ $\mathcal{O}\left(\alpha^{2}\right)$. Moreover,

$$
\begin{aligned}
& 4 \alpha^{\frac{1}{2}} \operatorname{Re}\left\langle P_{f} \cdot A^{-} \pi^{2} \Psi_{1}^{\perp}, \Phi_{*}^{u_{\alpha}}\right\rangle \\
& =4 \alpha^{\frac{3}{2}} \operatorname{Re}\left\langle P_{f} \cdot A^{-}\left(\kappa_{2} \Phi_{*}^{2} \pi^{0} \Psi_{1}^{\perp}+\sum_{i} \kappa_{2, i}\left(H_{f}+P_{f}^{2}\right)^{-1} W_{i} \frac{\partial u_{\alpha}}{\partial x_{i}}\right), \Phi_{*}^{u_{\alpha}}\right\rangle \\
& =4 \alpha^{\frac{3}{2}} \operatorname{Re}\left\langle P_{f} \cdot A^{-} \sum_{i} \kappa_{2, i}\left(H_{f}+P_{f}^{2}\right)^{-1} W_{i} \frac{\partial u_{\alpha}}{\partial x_{i}}, \Phi_{*}^{u_{\alpha}}\right\rangle \\
& =\frac{2}{3} \alpha^{4} \operatorname{Re} \sum_{i} \kappa_{2, i}\left\langle P_{f} \cdot A^{-}\left(H_{f}+P_{f}^{2}\right)^{-1} W_{i},\left(H_{f}+P_{f}^{2}\right)^{-1}\left(A^{+}\right)^{i} \Omega_{f}\right\rangle
\end{aligned}
$$

where we used (207) of Lemma C.2 in the second equality.

Collecting (176)-(199) concludes the proof. 
Lemma B.8. We have

$$
\begin{aligned}
& \left\langle\Psi_{1}^{\perp}, \Psi_{1}^{\perp}\right\rangle_{\sharp}+2 \alpha \operatorname{Re}\left\langle A^{-} \cdot A^{-} \Psi_{1}^{\perp}, \Psi_{1}^{\perp}\right\rangle \geq \Sigma_{0}\left(\left\|\pi^{0} \Psi_{1}^{\perp}\right\|^{2}+\left\|\pi^{1} \Psi_{1}^{\perp}\right\|^{2}\right) \\
& +\frac{\alpha^{4}}{12} \sum_{i=1}^{3}\left|\kappa_{2, i}\right|^{2}\left\|\left(H_{f}+P_{f}^{2}\right)^{-1} W_{i}\right\|_{*}^{2}+\alpha^{2}\left|\kappa_{3}+1\right|^{2}\left\|\Phi_{*}^{2}\right\|_{*}^{2}\left\|\Phi_{\sharp}^{u_{\alpha}}\right\|^{2} \\
& \quad+\left\|\pi^{1} \Psi_{1}^{\perp}\right\|_{\sharp}^{2}+\left\|\pi^{0} \Psi_{1}^{\perp}\right\|_{\sharp}^{2}+o\left(\alpha^{5} \log \alpha^{-1}\right) .
\end{aligned}
$$

Proof. Obviously, we have

$$
\left\langle\Psi_{1}^{\perp}, \Psi_{1}^{\perp}\right\rangle_{\sharp}=\sum_{i=0}^{3}\left\langle\pi^{i} \Psi_{1}^{\perp}, \pi^{i} \Psi_{1}^{\perp}\right\rangle_{\sharp},
$$

and using Lemma C.2

$$
\left\langle\pi^{2} \Psi_{1}^{\perp}, \pi^{2} \Psi_{1}^{\perp}\right\rangle_{\sharp}=\alpha^{2}\left|\kappa_{2}\right|^{2}\left\|\Phi_{*}^{2} \pi^{0} \Psi_{1}^{\perp}\right\|_{\sharp}^{2}+\alpha^{2} \sum_{i=1}^{3}\left|\kappa_{2, i}\right|^{2}\left\|\left(H_{f}+P_{f}^{2}\right)^{-1} W_{i} \frac{\partial u_{\alpha}}{\partial x_{i}}\right\|_{\sharp}^{2},
$$

Moreover, from the inequality $\left\|\Phi_{*}^{2} \pi^{0} \Psi_{1}^{\perp}\right\|_{\sharp}^{2}>\left\|\Phi_{*}^{2}\right\|_{*}^{2}\left\|\pi^{0} \Psi_{1}^{\perp}\right\|^{2}$ we obtain

$$
\begin{aligned}
& \alpha^{2}\left|\kappa_{2}\right|^{2}\left\|\Phi_{*}^{2} \pi^{0} \Psi_{1}^{\perp}\right\|_{\sharp}^{2}+2 \alpha \operatorname{Re}\left\langle A^{-} \cdot A^{-} \pi^{2} \Psi_{1}^{\perp}, \pi^{0} \Psi_{1}^{\perp}\right\rangle \\
& \geq \alpha^{2}\left|\kappa_{2}\right|^{2}\left\|\Phi_{*}^{2}\right\|_{*}^{2}\left\|\pi^{0} \Psi_{1}^{\perp}\right\|^{2}+2 \alpha \operatorname{Re}\left\langle A^{-} . A^{-} \pi^{2} \Psi_{1}^{\perp}, \pi^{0} \Psi_{1}^{\perp}\right\rangle \\
& =\alpha^{2}\left|\kappa_{2}\right|^{2}\left\|\Phi_{*}^{2}\right\|_{*}^{2}\left\|\pi^{0} \Psi_{1}^{\perp}\right\|^{2}-2 \alpha^{2} \operatorname{Re} \kappa_{2}\left\|\Phi_{*}^{2}\right\|_{*}^{2}\left\|\pi^{0} \Psi_{1}^{\perp}\right\|^{2} \\
& \quad+2 \alpha \operatorname{Re} \sum_{i=1}^{3} \alpha\left\langle A^{-} \cdot A^{-} \kappa_{2, i}\left(H_{f}+P_{f}^{2}\right)^{-1} W_{i} \frac{\partial u_{\alpha}}{\partial x_{i}}, \pi^{0} \Psi_{1}^{\perp}\right\rangle \\
& \quad \geq \Sigma_{0}\left\|\pi^{0} \Psi_{1}^{\perp}\right\|^{2}+\left|\kappa_{2}-1\right|^{2} c \alpha^{2}\left\|\pi^{0} \Psi_{1}^{\perp}\right\|^{2}+\mathcal{O}\left(\alpha^{5}\right) \geq \Sigma_{0}\left\|\pi^{0} \Psi_{1}^{\perp}\right\|^{2}+\mathcal{O}\left(\alpha^{5}\right) .
\end{aligned}
$$

where we used $\Sigma_{0}=-\alpha^{2}\left\|\Phi_{*}^{2}\right\|_{*}^{2}+\mathcal{O}\left(\alpha^{3}\right)$ and $\left\langle A^{-} \cdot A^{-}\left(H_{f}+P_{f}^{2}\right)^{-1} W_{i}, \pi^{0} \Psi_{1}^{\perp}\right\rangle=$ $-\overline{\pi^{0} \Psi_{1}^{\perp}}\left\langle W_{i}, \Phi_{*}^{2}\right\rangle=0$.

Similarly, $\left\|\pi^{3} \Psi_{1}^{\perp}\right\|_{\sharp}>\left\|\pi^{3} \Psi_{1}^{\perp}\right\|_{*}$ yields

$$
\begin{aligned}
&\left\|\pi^{3} \Psi_{1}^{\perp}\right\|_{\sharp}^{2}+2 \alpha \operatorname{Re}\left\langle A^{-} \cdot A^{-} \pi^{3} \Psi_{1}^{\perp}, \pi^{1} \Psi_{1}^{\perp}\right\rangle \\
& \geq \alpha^{2}\left|\kappa_{3}\right|^{2}\left\|\left(H_{f}+P_{f}^{2}\right)^{-1} A^{+} \cdot A^{+} \Phi_{\sharp}^{u_{\alpha}}\right\|_{*}^{2} \\
&+2 \operatorname{Re} \alpha^{2} \kappa_{3}\left\langle A^{-} \cdot A^{-}\left(H_{f}+P_{f}^{2}\right)^{-1} A^{+} \cdot A^{+} \Phi_{\sharp}^{u_{\alpha}}, \kappa_{1} \Phi_{\sharp}^{u_{\alpha}}\right\rangle \\
& \geq-\alpha^{2}\left\|\left(H_{f}+P_{f}^{2}\right)^{-1} A^{+} \cdot A^{+} \kappa_{1} \Phi_{\sharp}^{u_{\alpha}}\right\|_{*}^{2} \\
&+\left|\kappa_{3}+1\right|^{2} \alpha^{2}\left\|\left(H_{f}+P_{f}^{2}\right)^{-1} A^{+} \cdot A^{+} \Phi_{\sharp}^{u_{\alpha}}\right\|_{*}^{2} \\
& \geq \Sigma_{0}\left\|\pi^{1} \Psi_{1}^{\perp}\right\|^{2}+\alpha^{2}\left|\kappa_{3}+1\right|^{2}\left\|\Phi_{*}^{2}\right\|_{*}^{2}\left\|\Phi_{\sharp}^{u_{\alpha}}\right\|^{2}+o\left(\alpha^{5} \log \alpha^{-1}\right),
\end{aligned}
$$

where in the last inequality, we used (222) from Lemma C.6, $\kappa_{1}=1+\mathcal{O}\left(\alpha^{\frac{1}{2}}\right)$ from Lemma $6.1,\left\|\Phi_{\sharp}^{u_{\alpha}}\right\|^{2}=\mathcal{O}\left(\alpha^{3} \log \alpha^{-1}\right)$ from Lemma C.4 and $-\alpha^{2}\left\|\Phi_{*}^{2}\right\|_{*}^{2}=$ $\Sigma_{0}+\mathcal{O}\left(\alpha^{3}\right)$. 
The second term on the right-hand side of (181) is estimated as

$$
\begin{aligned}
& \alpha^{2} \sum_{i=1}^{3}\left|\kappa_{2, i}\right|^{2}\left\|\left(H_{f}+P_{f}^{2}\right)^{-1} W_{i} \frac{\partial u_{\alpha}}{\partial x_{i}}\right\|_{\sharp}^{2} \geq \alpha^{2} \sum_{i=1}^{3}\left|\kappa_{2, i}\right|^{2}\left\|\left(H_{f}+P_{f}^{2}\right)^{-1} W_{i} \frac{\partial u_{\alpha}}{\partial x_{i}}\right\|_{*}^{2} \\
& =\alpha^{2} \frac{1}{3}\left\|\nabla u_{\alpha}\right\|^{2} \sum_{i=1}^{3}\left|\kappa_{2, i}\right|^{2}\left\|\left(H_{f}+P_{f}^{2}\right)^{-1} W_{i}\right\|_{*}^{2} \\
& =\frac{\alpha^{4}}{12} \sum_{i=1}^{3}\left|\kappa_{2, i}\right|^{2}\left\|\left(H_{f}+P_{f}^{2}\right)^{-1} W_{i}\right\|_{*}^{2} .
\end{aligned}
$$

Collecting (180)-(184) concludes the proof.

Proposition B.2. We have

$$
\begin{aligned}
\langle(H+ & \left.\left.e_{0}\right) \Psi_{1}^{\perp}, \Psi_{1}^{\perp}\right\rangle \\
\geq & -4 \alpha\left\|\left(h_{\alpha}+e_{0}\right)^{-\frac{1}{2}} Q_{\alpha}^{\perp} P . A^{-} \Phi_{*}^{u_{\alpha}}\right\|^{2}+\left\|\left(h_{\alpha}+e_{0}\right)^{\frac{1}{2}} \pi^{0}\left(\Psi_{1}^{\perp}\right)^{a}\right\|^{2} \\
& +c_{0} \alpha\left\|\left(h_{\alpha}+e_{0}\right)^{\frac{1}{2}} \pi^{0}\left(\Psi_{1}^{\perp}\right)^{s}\right\|^{2}+\Sigma_{0}\left(\left\|\pi^{0} \Psi_{1}^{\perp}\right\|^{2}+\left\|\pi^{1} \Psi_{1}^{\perp}\right\|^{2}\right) \\
& +\frac{\alpha^{4}}{12} \sum_{i=1}^{3}\left|\kappa_{2, i}\right|^{2}\left\|\left(H_{f}+P_{f}^{2}\right)^{-1} W_{i}\right\|_{*}^{2}+4 \alpha^{\frac{1}{2}} \operatorname{Re}\left\langle\pi^{2} \Psi_{1}^{\perp}, A^{+} . P_{f} \Phi_{\sharp}^{u_{\alpha}}\right\rangle \\
& +\left\|\kappa_{1} \Phi_{\sharp}^{u_{\alpha}}\right\|_{\sharp}^{2}+2 \alpha\left\|A^{-} \Phi_{*}^{u_{\alpha}}\right\|^{2}+\alpha^{2}\left|\kappa_{3}+1\right|^{2}\left\|\Phi_{*}^{2}\right\|_{*}^{2}\left\|\Phi_{\sharp}^{u_{\alpha}}\right\|^{2} \\
& -\epsilon M\left[\Psi_{2}^{\perp}\right]-\left|\kappa_{1}-1\right| c \alpha^{4}+o\left(\alpha^{5} \log \alpha^{-1}\right),
\end{aligned}
$$

where $c_{0}$ is the same positive constant as in Proposition B.1 and

- $Q_{\alpha}^{\perp}$ is the orthogonal projection onto $\operatorname{Span}\left(u_{\alpha}\right)^{\perp}$,

- $\left(\Psi_{1}^{\perp}\right)^{a}$ is the odd part of $\pi^{0} \Psi_{1}^{\perp}$.

Proof. Collecting Lemmata B.6, B.7, and B.8 yields

$$
\begin{aligned}
\langle(H & \left.\left.+e_{0}\right) \Psi_{1}^{\perp}, \Psi_{1}^{\perp}\right\rangle \geq-4 \alpha^{\frac{1}{2}} \operatorname{Re}\left\langle A^{-} \Phi_{\sharp}^{u_{\alpha}}, P \pi^{0}\left(\Psi_{1}^{\perp}\right)^{s}\right\rangle \\
& +4 \alpha \operatorname{Re}\left\langle\pi^{2} \Psi_{1}^{\perp}, A^{+} . P_{f} \Phi_{\sharp}^{u_{\alpha}}\right\rangle \\
& +\Sigma_{0}\left(\left\|\pi^{0} \Psi_{1}^{\perp}\right\|^{2}+\left\|\pi^{1} \Psi_{1}^{\perp}\right\|^{2}\right)+\alpha^{2}\left|\kappa_{3}+1\right|^{2}\left\|\Phi_{*}^{2}\right\|_{*}^{2}\left\|\Phi_{\sharp}^{u_{\alpha}}\right\|^{2} \\
& +\left\|\left(h_{\alpha}+e_{0}\right)^{\frac{1}{2}} \pi^{0} \Psi_{1}^{\perp}\right\|^{2}+\left\|\pi^{1} \Psi_{1}^{\perp}\right\|_{\sharp}^{2}+2 \alpha\left\|A^{-} \kappa_{1} \Phi_{\sharp}^{u_{\alpha}}\right\|^{2}-\left|\kappa_{1}-1\right| c \alpha^{4} \\
& +\frac{\alpha^{4}}{12} \sum_{i=1}^{3}\left|\kappa_{2, i}\right|^{2}\left\|\left(H_{f}+P_{f}^{2}\right)^{-1} W_{i}\right\|_{*}^{2}-\epsilon M\left[\Psi_{2}^{\perp}\right]+o\left(\alpha^{5} \log \alpha^{-1}\right) .
\end{aligned}
$$

Obviously,

$\left\|\left(h_{\alpha}+e_{0}\right)^{\frac{1}{2}} \pi^{0} \Psi_{1}^{\perp}\right\|^{2}=\left\|\left(h_{\alpha}+e_{0}\right)^{\frac{1}{2}} \pi^{0}\left(\Psi_{1}^{\perp}\right)^{s}\right\|^{2}+\left\|\left(h_{\alpha}+e_{0}\right)^{\frac{1}{2}} \pi^{0}\left(\Psi_{1}^{\perp}\right)^{a}\right\|^{2}$.

As before, we write $\pi^{0} \Psi_{1}^{\perp}$ as the sum of its odd part $\left(\Psi_{1}^{\perp}\right)^{a}$ and its even part $\left(\Psi_{1}^{\perp}\right)^{s}$. Since $\pi^{0} \Psi_{1}^{\perp}$ is orthogonal to $u_{\alpha}$ by definition of $\Psi^{\perp}$, and $\left(\Psi_{1}^{\perp}\right)^{a}$ is orthogonal to $u_{\alpha}$ by symmetry of $u_{\alpha}$, we also have $\left(\Psi_{1}^{\perp}\right)^{s}$ orthogonal to $u_{\alpha}$. Therefore, one can replace $\pi^{0}\left(\Psi_{1}^{\perp}\right)^{s}$ by $Q_{\alpha}^{\perp} \pi^{0}\left(\Psi_{1}^{\perp}\right)^{s}$ in (186) and (187). Thus, 
as the next step, given a constant $c_{0}>0$, we minimize

$$
\begin{aligned}
& \left(1-c_{0} \alpha\right)\left\|\left(h_{\alpha}+e_{0}\right)^{\frac{1}{2}} Q_{\alpha}^{\perp} \pi^{0}\left(\Psi_{1}^{\perp}\right)^{s}\right\|^{2}-4 \alpha^{\frac{1}{2}} \operatorname{Re}\left\langle Q_{\alpha}^{\perp} P \cdot A^{-} \Phi_{\sharp}^{u_{\alpha}}, Q_{\alpha}^{\perp} \pi^{0}\left(\Psi_{1}^{\perp}\right)^{s}\right\rangle \\
& \geq-\frac{4 \alpha}{1-c_{0} \alpha}\left\|\left(h_{\alpha}+e_{0}\right)^{-\frac{1}{2}} Q_{\alpha}^{\perp} P . A^{-} \Phi_{\sharp}^{u_{\alpha}}\right\|^{2} .
\end{aligned}
$$

Obviously,

$$
\begin{aligned}
& -\frac{4 \alpha}{1-c_{0} \alpha}\left\|\left(h_{\alpha}+e_{0}\right)^{-\frac{1}{2}} Q_{\alpha}^{\perp} P \cdot A^{-} \Phi_{\sharp}^{u_{\alpha}}\right\|^{2} \\
& \geq-\frac{4(1+\alpha) \alpha}{1-c_{0} \alpha}\left\|\left(h_{\alpha}+e_{0}\right)^{-\frac{1}{2}} Q_{\alpha}^{\perp} P \cdot A^{-} \Phi_{*}^{u_{\alpha}}\right\|^{2} \\
& \quad-\frac{4\left(1+\alpha^{-1}\right) \alpha}{1-c_{0} \alpha}\left\|\left(h_{\alpha}+e_{0}\right)^{-\frac{1}{2}} Q_{\alpha}^{\perp} P \cdot A^{-}\left(\Phi_{\sharp}^{u_{\alpha}}-\Phi_{*}^{u_{\alpha}}\right)\right\|^{2} .
\end{aligned}
$$

There exist $\gamma_{1}$ and $\gamma_{2}$ positive, independent of $\alpha$, such that

$$
Q_{\alpha}^{\perp}\left(h_{\alpha}+e_{0}\right)^{-1} Q_{\alpha}^{\perp} \leq\left(\gamma_{1} P^{2}+\gamma_{2} \alpha^{2}\right)^{-1},
$$

and thus $P Q_{\alpha}^{\perp}\left(h_{\alpha}+e_{0}\right)^{-1} Q_{\alpha}^{\perp} P$ is a bounded operator. In addition, since $\left\|A^{-}\left(\Phi_{\sharp}^{u_{\alpha}}-\Phi_{*}^{u_{\alpha}}\right)\right\|^{2}=\mathcal{O}\left(\alpha^{7} \log \alpha^{-1}\right)$ (Lemma C.4), this shows that

$$
\frac{4\left(1+\alpha^{-1}\right) \alpha}{1-c_{0} \alpha}\left\|\left(h_{\alpha}+e_{0}\right)^{-\frac{1}{2}} Q_{\alpha}^{\perp} P . A^{-}\left(\Phi_{\sharp}^{u_{\alpha}}-\Phi_{*}^{u_{\alpha}}\right)\right\|^{2}=\mathcal{O}\left(\alpha^{6} \log \alpha^{-1}\right) .
$$

In addition, using $\left\|A^{-} \Phi_{*}^{u_{\alpha}}\right\| \leq\left\|A^{-}\left(\Phi_{*}^{u_{\alpha}}-\Phi_{\sharp}^{u_{\alpha}}\right)\right\|+\left\|A^{-} \Phi_{\sharp}^{u_{\alpha}}\right\| \leq c \alpha^{\frac{3}{2}}$ (Lemma C.4 and Lemma C.5), and the fact that $P Q_{\alpha}^{\perp}\left(h_{\alpha}+e_{0}\right)^{-1} Q_{\alpha}^{\perp} P$ is bounded, yields

$$
\begin{aligned}
- & \frac{(1+\alpha) \alpha}{1-c_{0} \alpha}\left\|\left(h_{\alpha}+e_{0}\right)^{-\frac{1}{2}} Q_{\alpha}^{\perp} P \cdot A^{-} \Phi_{*}^{u_{\alpha}}\right\|^{2} \\
& =-4\left\|\left(h_{\alpha}+e_{0}\right)^{-\frac{1}{2}} Q_{\alpha}^{\perp} P . A^{-} \Phi_{*}^{u_{\alpha}}\right\|^{2}+\mathcal{O}\left(\alpha^{5}\right) .
\end{aligned}
$$

Collecting (189)-(191), one gets

$$
\begin{aligned}
& -\frac{4 \alpha}{1-c_{0} \alpha}\left\|\left(h_{\alpha}+e_{0}\right)^{-\frac{1}{2}} Q_{\alpha}^{\perp} P \cdot A^{-} \Phi_{\sharp}^{u_{\alpha}}\right\|^{2} \\
& \quad \geq-4\left\|\left(h_{\alpha}+e_{0}\right)^{-\frac{1}{2}} Q_{\alpha}^{\perp} P . A^{-} \Phi_{*}^{u_{\alpha}}\right\|^{2}+\mathcal{O}\left(\alpha^{5}\right) .
\end{aligned}
$$

Finally, using $\left\|A^{-} \Phi_{*}^{u_{\alpha}}\right\|^{2}=\mathcal{O}\left(\alpha^{3}\right)$, we obtain

$$
2 \alpha\left|\kappa_{1}\right|^{2}\left\|A^{-} \Phi_{*}^{u_{\alpha}}\right\|^{2} \geq 2 \alpha\left\|A^{-} \Phi_{*}^{u_{\alpha}}\right\|^{2}-c\left|\kappa_{1}-1\right| \alpha^{4} .
$$

Substituting (187), (188), (192) and (193) into (186) concludes the proof.

We can now collect the above results to prove Proposition 6.2. 


\section{B.3. Concluding the Proof of Proposition 6.2}

Collecting the results of Proposition B.1, Lemmata B.1-B.5 and Proposition B.2 yields directly the following bound:

$$
\begin{aligned}
&\left\langle H \Psi^{\perp}, \Psi^{\perp}\right\rangle \\
& \geq\left\langle H \Psi_{2}^{\perp}, \Psi_{2}^{\perp}\right\rangle-\epsilon M\left[\Psi_{2}^{\perp}\right]-c\left|\kappa_{2}\right|^{2} \alpha^{4}\left\|\pi^{0} \Psi_{1}^{\perp}\right\|^{2}-c \alpha^{6} \log \alpha^{-1}\left|\kappa_{3}\right|^{2}-e_{0}\left\|\Psi_{1}^{\perp}\right\|^{2} \\
&-4 \alpha\left\|\left(h_{\alpha}+e_{0}\right)^{-\frac{1}{2}} Q_{\alpha}^{\perp} P . A^{-} \Phi_{*}^{u_{\alpha}}\right\|^{2}+\left(1-c_{0} \alpha\right)\left\|\left(h_{\alpha}+e_{0}\right)^{\frac{1}{2}} \pi^{0}\left(\Psi_{1}^{\perp}\right)^{a}\right\|^{2} \\
&+\Sigma_{0}\left(\left\|\pi^{0} \Psi_{1}^{\perp}\right\|^{2}+\left\|\pi^{1} \Psi_{1}^{\perp}\right\|^{2}\right)+\frac{\alpha^{4}}{12} \sum_{i=1}^{3}\left|\kappa_{2, i}\right|^{2}\left\|\left(H_{f}+P_{f}^{2}\right)^{-1} W_{i}\right\|_{*}^{2} \\
&+4 \alpha^{\frac{1}{2}} \operatorname{Re}\left\langle\pi^{2} \Psi_{2}^{\perp}, A^{+} . P_{f} \Phi_{*}^{u_{\alpha}}\right\rangle+4 \alpha^{\frac{1}{2}} \operatorname{Re}\left\langle\pi^{2} \Psi_{1}^{\perp}, A^{+} . P_{f} \Phi_{\sharp}^{u_{\alpha}}\right\rangle \\
&+\left|\kappa_{1}\right|^{2}\left\|\Phi_{\sharp}^{u_{\alpha}}\right\|_{\sharp}^{2}+2 \alpha\left\|A^{-} \Phi_{*}^{u_{\alpha}}\right\|^{2} \\
&+\alpha^{2}\left|\kappa_{3}+1\right|^{2}\left\|\Phi_{*}^{2}\right\|_{*}^{2}\left\|\Phi_{\sharp}^{u_{\alpha}}\right\|^{2}-\left|\kappa_{1}-1\right| c \alpha^{4}+o\left(\alpha^{5} \log \alpha^{-1}\right) .
\end{aligned}
$$

Comparing this expression with the statement of the Proposition, we see that it suffices to show that

$$
\begin{aligned}
-\epsilon & M\left[\Psi_{2}^{\perp}\right]-c\left|\kappa_{2}\right|^{2} \alpha^{4}\left\|\pi^{0} \Psi_{1}^{\perp}\right\|^{2}-c \alpha^{6} \log \alpha^{-1}\left|\kappa_{3}\right|^{2}-e_{0}\left\|\Psi_{1}^{\perp}\right\|^{2}+\left\langle H \Psi_{2}^{\perp}, \Psi_{2}^{\perp}\right\rangle \\
& +\Sigma_{0}\left(\left\|\pi^{0} \Psi_{1}^{\perp}\right\|^{2}+\left\|\pi^{1} \Psi_{1}^{\perp}\right\|^{2}\right)+\alpha^{2}\left|\kappa_{3}+1\right|^{2}\left\|\Phi_{*}^{2}\right\|_{*}^{2}\left\|\Phi_{\sharp}^{u_{\alpha}}\right\|^{2} \\
\geq & \left(\Sigma_{0}-e_{0}\right)\left\|\Psi^{\perp}\right\|^{2}+(1-\epsilon) M\left[\Psi_{2}^{\perp}\right]+\frac{\left|\kappa_{3}+1\right|^{2}}{2} \alpha^{2}\left\|\Phi_{*}^{2}\right\|_{*}^{2}\left\|\Phi_{\sharp}^{u_{\alpha}}\right\|^{2}+\mathcal{O}\left(\alpha^{5}\right) .
\end{aligned}
$$

Using from Corollary 4.2 that $\left\langle H \Psi_{2}^{\perp}, \Psi_{2}^{\perp}\right\rangle \geq\left(\Sigma_{0}-e_{0}\right)\left\|\Psi_{2}^{\perp}\right\|^{2}+M\left[\Psi_{2}^{\perp}\right]$, we first estimate the following terms in (195):

$$
\begin{aligned}
\Sigma_{0} & \left(\left\|\pi^{0} \Psi_{1}^{\perp}\right\|^{2}+\left\|\pi^{1} \Psi_{1}^{\perp}\right\|^{2}\right)-e_{0}\left\|\Psi_{1}^{\perp}\right\|^{2}+\left\langle H \Psi_{2}^{\perp}, \Psi_{2}^{\perp}\right\rangle-\epsilon M\left[\Psi_{2}^{\perp}\right] \\
\geq & (1-\epsilon) M\left[\Psi_{2}^{\perp}\right]+\left(\Sigma_{0}-e_{0}\right)\left(\left\|\Psi_{1}^{\perp}\right\|^{2}+\left\|\Psi_{2}^{\perp}\right\|^{2}\right)-\Sigma_{0}\left\|\pi^{n \geq 2} \Psi_{1}^{\perp}\right\|^{2} \\
\geq & (1-\epsilon) M\left[\Psi_{2}^{\perp}\right]+\left(\Sigma_{0}-e_{0}\right)\left\|\Psi^{\perp}\right\|^{2} \\
& -\left(\Sigma_{0}-e_{0}\right)\left(\left\|\Psi^{\perp}\right\|^{2}-\left\|\Psi_{1}^{\perp}\right\|^{2}-\left\|\Psi_{2}^{\perp}\right\|^{2}\right)-\Sigma_{0}\left\|\pi^{n \geq 2} \Psi_{1}^{\perp}\right\|^{2} .
\end{aligned}
$$

We have obviously

$$
\begin{aligned}
& \left\|\Psi^{\perp}\right\|^{2}-\left\|\Psi_{1}^{\perp}\right\|^{2}-\left\|\Psi_{2}^{\perp}\right\|^{2} \\
& \quad=2 \operatorname{Re}\left(\left\langle\pi^{1} \Psi_{1}^{\perp}, \pi^{1} \Psi_{2}^{\perp}\right\rangle+\left\langle\pi^{2} \Psi_{1}^{\perp}, \pi^{2} \Psi_{2}^{\perp}\right\rangle+\left\langle\pi^{3} \Psi_{1}^{\perp}, \pi^{3} \Psi_{2}^{\perp}\right\rangle\right) .
\end{aligned}
$$

Since $\left|\Sigma_{0}-e_{0}\right| \leq c \alpha^{2}$, by definition of $\pi^{3} \Psi_{1}^{\perp}$ and Lemma C.6, we obtain

$$
\begin{aligned}
& \left|\left(\Sigma_{0}-e_{0}\right) 2 \operatorname{Re}\left\langle\pi^{3} \Psi_{1}^{\perp}, \pi^{3} \Psi_{2}^{\perp}\right\rangle\right| \\
& \quad \leq \epsilon \alpha^{2}\left\|\pi^{3} \Psi_{2}^{\perp}\right\|^{2}+c \alpha^{4}\left|\kappa_{3}\right|^{2}\left\|\left(H_{f}+P_{f}^{2}\right)^{-1} A^{+} . A^{+} \Phi_{\sharp}^{u_{\alpha}}\right\|^{2} \\
& \quad \leq \epsilon \alpha^{2}\left\|\pi^{3} \Psi_{2}^{\perp}\right\|^{2}+c\left|\kappa_{3}\right|^{2} \alpha^{7} \log \alpha^{-1} .
\end{aligned}
$$


Similarly, for the two-photon sector, we find

$$
\begin{aligned}
& \left|\left(\Sigma_{0}-e_{0}\right)\left\langle\pi^{2} \Psi_{1}^{\perp}, \pi^{2} \Psi_{2}^{\perp}\right\rangle\right| \\
& \quad \leq \epsilon \alpha^{2}\left\|\pi^{2} \Psi_{2}^{\perp}\right\|^{2}+c \alpha^{4}\left|\kappa_{2}\right|^{2}\left\|\pi^{0} \Psi_{1}^{\perp}\right\|^{2}+c \sum_{i=1}^{3}\left|\kappa_{2, i}\right|^{2} \alpha^{6} \\
& \quad=\epsilon \alpha^{2}\left\|\pi^{2} \Psi_{2}^{\perp}\right\|^{2}+\mathcal{O}\left(\alpha^{6}\right),
\end{aligned}
$$

where we used Lemma 6.1. For the term $\left\langle\pi^{1} \Psi_{1}^{\perp}, \pi^{1} \Psi_{2}^{\perp}\right\rangle$, one gets

$$
\begin{aligned}
& \left|\left(\Sigma_{0}-e_{0}\right)\left\langle\pi^{1} \Psi_{2}^{\perp}, \kappa_{1} \Phi_{\sharp}^{u_{\alpha}}\right\rangle\right|=\left|\left(\Sigma_{0}-e_{0}\right)\left(|k|^{\frac{1}{2}} \pi^{1} \Psi_{2}^{\perp},|k|^{-\frac{1}{2}} \kappa_{1} \Phi_{\sharp}^{u_{\alpha}}\right\rangle\right| \\
& \quad \leq \epsilon\left\|H_{f}^{\frac{1}{2}} \pi^{1} \Psi_{2}^{\perp}\right\|^{2}+c \alpha^{4}\left|\kappa_{1}\right|^{2}\left\||k|^{-\frac{1}{2}} \Phi_{\sharp}^{u_{\alpha}}\right\|^{2} \leq \epsilon\left\|H_{f}^{\frac{1}{2}} \pi^{1} \Psi_{2}^{\perp}\right\|^{2}+\mathcal{O}\left(\alpha^{5}\right),
\end{aligned}
$$

since $\left\||k|^{-\frac{1}{2}} \Phi_{\sharp}^{u_{\alpha}}\right\|^{2}=\mathcal{O}(\alpha)$ (Lemma C.4) and $\kappa_{1}=\mathcal{O}(1)$ (Lemma 6.1).

Collecting (197), (198), (199) and (200) yields

$$
\left|\Sigma_{0}-e_{0}\right|||\left|\Psi^{\perp}\left\|^{2}-\right\| \Psi_{1}^{\perp}\left\|^{2}-\right\| \Psi_{2}^{\perp} \|^{2}\right| \leq \epsilon M\left[\Psi_{2}^{\perp}\right]+c\left|\kappa_{3}\right|^{2} \alpha^{7} \log \alpha^{-1}+\mathcal{O}\left(\alpha^{5}\right) .
$$

Therefore, together with (196), one finds

$$
\begin{aligned}
& \Sigma_{0}\left(\left\|\pi^{0} \Psi_{1}^{\perp}\right\|^{2}+\left\|\pi^{1} \Psi_{1}^{\perp}\right\|^{2}\right)-e_{0}\left\|\Psi_{1}^{\perp}\right\|^{2}+\left\langle H \Psi_{2}^{\perp}, \Psi_{2}^{\perp}\right\rangle-\epsilon M\left[\Psi_{2}^{\perp}\right] \\
& \geq(1-2 \epsilon) M\left[\Psi_{2}^{\perp}\right]+\left(\Sigma_{0}-e_{0}\right)\left\|\Psi^{\perp}\right\|^{2} \\
& \quad-c\left|\kappa_{3}\right|^{2} \alpha^{7} \log \alpha^{-1}-\Sigma_{0}\left\|\pi^{n \geq 2} \Psi_{1}^{\perp}\right\|^{2}+\mathcal{O}\left(\alpha^{5}\right),
\end{aligned}
$$

By definition of $\Psi_{1}^{\perp}$ and using $\Sigma_{0}=\mathcal{O}\left(\alpha^{2}\right),\left|\kappa_{2}\right|\left\|\pi^{0} \Psi_{1}^{\perp}\right\|=\mathcal{O}(\alpha)$ (Lemma 6.1) and Inequality (223) of Lemma C.6, we straightforwardly obtain

$$
\Sigma_{0}\left\|\pi^{n \geq 2} \Psi_{1}^{\perp}\right\|^{2} \leq c \alpha^{6}+c\left|\kappa_{3}\right|^{2} \alpha^{7} \log \alpha^{-1} .
$$

Substituting this in (202) yields

$$
\begin{aligned}
& \Sigma_{0}\left(\left\|\pi^{0} \Psi_{1}^{\perp}\right\|^{2}+\left\|\pi^{1} \Psi_{1}^{\perp}\right\|^{2}\right)-e_{0}\left\|\Psi_{1}^{\perp}\right\|^{2}+\left\langle H \Psi_{2}^{\perp}, \Psi_{2}^{\perp}\right\rangle-\epsilon M\left[\Psi_{2}^{\perp}\right] \\
& \quad \geq(1-2 \epsilon) M\left[\Psi_{2}^{\perp}\right]+\left(\Sigma_{0}-e_{0}\right)\left\|\Psi^{\perp}\right\|^{2}-2 c\left|\kappa_{3}\right|^{2} \alpha^{7} \log \alpha^{-1}+\mathcal{O}\left(\alpha^{5}\right) .
\end{aligned}
$$

To conclude the proof of (195), and thus of the Proposition, we first note that according to Lemma 6.1 ,

$$
-c\left|\kappa_{2}\right|^{2} \alpha^{4}\left\|\pi^{0} \Psi_{1}^{\perp}\right\|^{2}=\mathcal{O}\left(\alpha^{6}\right) .
$$

Similarly, taking into account that $\left\|\Phi_{\sharp}^{u_{\alpha}}\right\|^{2}=c \alpha^{3} \log \alpha^{-1}$ (see (209) in Lemma C.4), we get for some $c_{2}>0$,

$$
\begin{aligned}
& \alpha^{2} \frac{\left|\kappa_{3}+1\right|^{2}}{2}\left\|\Phi_{*}^{2}\right\|_{*}^{2}\left\|\Phi_{\sharp}^{u_{\alpha}}\right\|^{2}-c \alpha^{6} \log \alpha^{-1}\left|\kappa_{3}\right|^{2}-2 c \alpha^{7} \log \alpha^{-1}\left|\kappa_{3}\right|^{2} \\
& \geq-c_{2} \alpha^{6} \log \alpha^{-1} .
\end{aligned}
$$

Collecting (203), (204) and (205) yields the bound (195), and thus concludes the proof of the Proposition 6.2. 


\section{Appendix C. Proof of Theorem 2.1: Auxiliary Lemmata}

The proof of Theorem 2.1 uses Lemmata C.2-C.7 below.

Lemma C.1. Let $\Psi_{0}$ be the ground state of $T(0)$, with $\left|\pi^{0} \Psi_{0}\right|=1$. Then we have

$$
\begin{array}{r}
\Psi_{0}=\Omega_{f}+2 \eta_{1} \alpha^{\frac{3}{2}} \Phi_{*}^{1}+\eta_{2} \alpha \Phi_{*}^{2}+2 \eta_{3} \alpha^{\frac{3}{2}} \Phi_{*}^{3}+\Delta_{*}^{0} \\
\text { with }\left\langle\Delta_{*}^{0}, \Phi_{*}^{i}\right\rangle_{*}=0(i=1,2,3), \pi^{0} \Delta_{*}^{0}=0
\end{array}
$$

and

$$
\left\|\Delta_{*}^{0}\right\|^{2}=\mathcal{O}\left(\alpha^{3}\right) .
$$

Proof. It follows from a similar argument as in [9, Proposition 5.1] that

$$
\left\|a_{\lambda}(k) \Delta_{*}^{0}\right\| \leq \frac{c \alpha}{|k|} .
$$

This yields

$$
\begin{aligned}
\left\|\Delta_{*}^{0}\right\|^{2} & \leq \int\left\|a_{\lambda}(k) \Delta_{*}^{0}\right\|^{2} \mathrm{~d} k \\
& \leq \int_{|k| \leq \alpha} \frac{c^{2} \alpha^{2}}{|k|^{2}} \mathrm{~d} k+\int_{|k|>\alpha} \frac{|k|\left\|a_{\lambda}(k) \Delta_{*}^{0}\right\|^{2} \chi_{\Lambda}(|k|)}{|k|} \mathrm{d} k \\
& \leq c^{2} \alpha^{3}+c^{\prime} \alpha^{-1}\left\|H_{f}^{\frac{1}{2}} \Delta_{*}^{0}\right\|^{2}=\mathcal{O}\left(\alpha^{3}\right),
\end{aligned}
$$

where in the last equality we used the estimate $\left\|\Delta_{*}^{0}\right\|_{*}^{2}=\left\|\left(H_{f}+P_{f}^{2}\right)^{\frac{1}{2}} \Delta_{*}^{0}\right\|^{2}=$ $\mathcal{O}\left(\alpha^{4}\right)$ proved in [7, Theorem 3.2].

Lemma C.2. We have

$$
P . A^{-} u_{\alpha} \Phi_{*}^{1}=0
$$

and

$$
\left\langle\Phi_{*}^{2}, \zeta\left(H_{f}, P_{f}^{2}\right) P_{f}^{i} \Phi_{*}^{2}\right\rangle=0, \quad \text { and }\left\langle\Phi_{*}^{2}, \zeta\left(H_{f}, P_{f}^{2}\right) W_{i}\right\rangle=0,
$$

for any function $\zeta$ for which the scalar products are defined. Similarly, we have

$$
\left\langle\Phi_{*}^{2} \pi^{0} \Psi_{1}^{\perp}, A^{+} . P_{f} \Phi_{*}^{u_{\alpha}}\right\rangle=0 .
$$

Proof. Straightforward computations using the symmetries of $A^{-} \Phi_{*}^{1}$ and $\Phi_{*}^{2}$.

Lemma C.3. We have

$$
P . A^{-} \Phi_{*}^{u_{\alpha}}=\sqrt{\alpha} a_{0} \Delta u_{\alpha}
$$

where

$$
a_{0}=\int \frac{k_{1}^{2}+k_{2}^{2}}{4 \pi^{2}|k|^{3}} \frac{2}{|k|^{2}+|k|} \chi_{\Lambda}(|k|) \mathrm{d} k_{1} \mathrm{~d} k_{2} \mathrm{~d} k_{3} .
$$

Proof. Straightforward computations using the symmetries of $A^{-} \Phi_{*}^{u_{\alpha}}$. 


\section{Lemma C.4.}

$$
\begin{gathered}
\forall \varphi \in \mathfrak{F}, \quad\left\langle P . P_{f} \pi^{1} \varphi u_{\alpha}, \Phi_{\sharp}^{u_{\alpha}}\right\rangle=0, \\
\left\|\Phi_{\sharp}^{u_{\alpha}}\right\|^{2}=\mathcal{O}\left(\alpha^{3} \log \alpha^{-1}\right), \\
\left\|\Phi_{\sharp}^{u_{\alpha}}\right\|_{*}^{2}=\mathcal{O}\left(\alpha^{3}\right), \\
\left\||k|^{-\frac{1}{2}} \Phi_{\sharp}^{u_{\alpha}}\right\|^{2}=\mathcal{O}(\alpha) . \\
\left\|P \Phi_{\sharp}^{u_{\alpha}}\right\|^{2}=\mathcal{O}\left(\alpha^{5} \log \alpha^{-1}\right), \\
\left\|P \Phi_{\sharp}^{u_{\alpha}}\right\|_{*}^{2}=\mathcal{O}\left(\alpha^{5}\right), \\
\left\||k|^{\frac{1}{6}} P \Phi_{\sharp}^{u_{\alpha}}\right\|^{2}=\mathcal{O}\left(\alpha^{5}\right) .
\end{gathered}
$$

Proof. The proof of (208) is as follows:

$$
\begin{aligned}
& \left\langle P . P_{f} \pi^{1} \varphi u_{\alpha}, \Phi_{\sharp}^{u_{\alpha}}\right\rangle \\
& =\int \sum_{i=1}^{3} k^{i} \frac{\partial u_{\alpha}}{\partial x_{i}} \varphi(k) \frac{1}{k^{2}+|k|+h_{\alpha}+e_{0}} \sum_{j=1}^{3} \sum_{\lambda=1,2} \frac{\epsilon_{\lambda}^{j}(k) \chi_{\Lambda}(|k|)}{2 \pi|k|^{\frac{1}{2}}} \frac{\overline{\partial u_{\alpha}}}{\partial x_{j}} \mathrm{~d} x \mathrm{~d} k \\
& =\sum_{i=1}^{3} \int \mathrm{d} k\left(\int \mathrm{d} x \frac{\partial u_{\alpha}}{\partial x_{i}} \frac{1}{k^{2}+|k|+h_{\alpha}+e_{0}} \frac{\partial u_{\alpha}}{\partial x_{i}}\right) \\
& \quad \times \sum_{\lambda=1,2} \frac{k^{i} \epsilon_{\lambda}^{i}(k) \chi_{\Lambda}(|k|)}{2 \pi|k|^{\frac{1}{2}}} \varphi(k) \mathrm{d} k=0
\end{aligned}
$$

using that the integral over $x$ is independent of the value of $i$, and since $k . \epsilon_{\lambda}(k)=0$.

To prove (211), we note that

$$
\begin{aligned}
\left\||k|^{-\frac{1}{2}} \Phi_{\sharp}^{u_{\alpha}}\right\|^{2} & \leq c \alpha \int\left|\frac{\chi_{\Lambda}(|k|)}{|k|\left(|k|+k^{2}+h_{\alpha}+e_{0}\right)} \nabla u_{\alpha}\right|^{2} \mathrm{~d} k \mathrm{~d} x \\
& \leq c \alpha^{3} \int \frac{\chi_{\Lambda}(|k|)^{2}}{|k|^{2}\left(|k|+\frac{3}{16} \alpha^{2}\right)^{2}} \mathrm{~d} k=\mathcal{O}(\alpha) .
\end{aligned}
$$

The proofs of (209) and (210) are similar, but simpler.

We next prove (212).

$$
\left\|P \Phi_{\sharp}^{u_{\alpha}}\right\|^{2}=c \alpha \sum_{i=1}^{3} \int\left\|P \frac{\chi_{\Lambda}(|k|)}{|k|^{\frac{1}{2}}\left(|k|+k^{2}+h_{\alpha}+e_{0}\right)} \frac{\partial u_{\alpha}}{\partial x_{i}}\right\|_{L^{2}\left(\mathbb{R}^{3}\right)}^{2} \mathrm{~d} k .
$$

The function $\partial u_{\alpha} / \partial x_{i}$ is odd. On the subspace of antisymmetric functions on $L^{2}\left(\mathbb{R}^{3}\right)$, one has that $-\left(1-\gamma_{0}\right) \Delta-\frac{\alpha}{|x|}>-e_{0}$ for some $\gamma_{0}>0$, which implies on this subspace that $h_{\alpha}+e_{0}>\gamma_{0} P^{2}$, and thus,

$$
P^{2}<\gamma_{0}^{-1}\left(h_{\alpha}+e_{0}\right) .
$$


The relation (217) yields, for all $k$

$$
\begin{aligned}
& \left\|P \frac{\chi_{\Lambda}(|k|)}{|k|^{\frac{1}{2}}\left(|k|+k^{2}+h_{\alpha}+e_{0}\right)} \frac{\partial u_{\alpha}}{\partial x_{i}}\right\|^{2} \\
& \quad \leq \gamma_{0}^{-1}\left\|\frac{\chi_{\Lambda}(|k|)}{|k|^{\frac{1}{2}}\left(|k|+k^{2}+h_{\alpha}+e_{0}\right)}\left(h_{\alpha}+e_{0}\right)^{\frac{1}{2}} \frac{\partial u_{\alpha}}{\partial x_{i}}\right\|^{2} \\
& \quad \leq \gamma_{0}^{-1} c \alpha^{4}\left(\frac{\chi_{\Lambda}(|k|)}{|k|^{\frac{1}{2}}\left(|k|+k^{2}+\frac{3}{16} \alpha^{2}\right)}\right)^{2} .
\end{aligned}
$$

Substituting (218) into (216) and integrating over $k$ proves (212).

The proof of (213) is similar.

Lemma C.5. We have

$$
\begin{aligned}
\left\|\Phi_{*}^{u_{\alpha}}\right\|_{*}^{2}-\left\|\Phi_{\sharp}^{u_{\alpha}}\right\|_{\sharp}^{2} & =\frac{1}{3 \pi}\left\|\left(h_{1}+\frac{1}{4}\right)^{\frac{1}{2}} \nabla u_{1}\right\|^{2} \alpha^{5} \log \alpha^{-1}+o\left(\alpha^{5} \log \alpha^{-1}\right), \\
\left\|\Phi_{*}^{u_{\alpha}}-\Phi_{\sharp}^{u_{\alpha}}\right\|_{*}^{2} & =\mathcal{O}\left(\alpha^{5}\right), \\
\left\|A^{-}\left(\Phi_{\sharp}^{u_{\alpha}}-\Phi_{*}^{u_{\alpha}}\right)\right\|^{2} & =\mathcal{O}\left(\alpha^{7} \log \alpha^{-1}\right) .
\end{aligned}
$$

Proof. We have

$$
\begin{aligned}
& \left\|\Phi_{*}^{u_{\alpha}}\right\|_{*}^{2}-\left\|\Phi_{\sharp}^{u_{\alpha}}\right\|_{\sharp}^{2} \\
& =\frac{\alpha^{5}}{(2 \pi)^{2}} \frac{4}{3}\left\|\frac{\chi_{\Lambda}(|k|)}{|k|^{\frac{1}{2}}\left(|k|+k^{2}\right)^{\frac{1}{2}}\left(|k|+k^{2}+\alpha^{2}\left(h_{1}+\frac{1}{4}\right)\right)^{\frac{1}{2}}}\left(h_{1}+\frac{1}{4}\right)^{\frac{1}{2}} \nabla u_{1}\right\|^{2},
\end{aligned}
$$

where the norm here is obviously taken on $L^{2}\left(\mathbb{R}^{3}, \mathrm{~d} x\right) \otimes L^{2}\left(\mathbb{R}^{3}, \mathrm{~d} k\right)$. Since $\left(h_{1}+\frac{1}{4}\right) \nabla u_{1} \in L^{2}$, it implies that for sufficiently large $c>0$ independent of $\alpha$,

$$
\left\|\chi\left(h_{1}>c\right)\left(h_{1}+\frac{1}{4}\right)^{\frac{1}{2}} \nabla u_{1}\right\|^{2}<\epsilon
$$

and

$$
\begin{aligned}
& \left\|\frac{\chi_{\Lambda}(|k|)}{|k|^{\frac{1}{2}}\left(|k|+k^{2}\right)^{\frac{1}{2}}\left(|k|+k^{2}+\alpha^{2}\left(h_{1}+\frac{1}{4}\right)\right)^{\frac{1}{2}}} \chi\left(h_{1}>c\right)\left(h_{1}+\frac{1}{4}\right)^{\frac{1}{2}} \nabla u_{1}\right\|^{2} \\
& \quad \leq \epsilon \int_{0}^{\infty} \frac{\chi_{\Lambda}^{2}(t)}{t+c \alpha^{2}} \mathrm{~d} t=\epsilon \log \alpha^{-1}+\mathcal{O}(1) .
\end{aligned}
$$


For the contribution of $\chi\left(h_{1} \leq c\right)\left(h_{1}+\frac{1}{4}\right)^{\frac{1}{2}} \nabla u_{1}$ in (219), the following inequalities hold:

$$
\begin{aligned}
& \left(\frac{1}{\pi} \log \alpha^{-1}+\mathcal{O}(1)\right) \frac{1}{3}\left\|\chi\left(h_{1}<c\right)\left(h_{1}+\frac{1}{4}\right)^{\frac{1}{2}} \nabla u_{1}\right\|^{2} \\
& =\frac{1}{(2 \pi)^{2}} \frac{1}{3} \\
& \left\|\frac{\chi_{\Lambda}(|k|)}{\left.|k|^{\frac{1}{2}}\left(|k|+k^{2}\right)^{\frac{1}{2}}\left(|k|+k^{2}+\left(c+\frac{1}{4}\right) \alpha^{2}\right)\right)^{\frac{1}{2}}} \chi\left(h_{1}<c\right)\left(h_{1}+\frac{1}{4}\right)^{\frac{1}{2}} \nabla u_{1}\right\|^{2} \\
& \leq \frac{1}{(2 \pi)^{2}} \frac{1}{3} \\
& \left\|\frac{\chi_{\Lambda}(|k|)}{\left.|k|^{\frac{1}{2}}\left(|k|+k^{2}\right)^{\frac{1}{2}}\left(|k|+k^{2}+\left(h_{1}+\frac{1}{4}\right) \alpha^{2}\right)\right)^{\frac{1}{2}}} \chi\left(h_{1}<c\right)\left(h_{1}+\frac{1}{4}\right)^{\frac{1}{2}} \nabla u_{1}\right\|^{2} \\
& \leq \frac{1}{(2 \pi)^{2}} \frac{1}{3}\left\|\frac{\chi_{\Lambda}(|k|)}{\left.|k|^{\frac{1}{2}}\left(|k|+k^{2}\right)^{\frac{1}{2}}\left(|k|+k^{2}+\frac{3}{16} \alpha^{2}\right)\right)^{\frac{1}{2}}} \chi\left(h_{1}<c\right)\left(h_{1}+\frac{1}{4}\right)^{\frac{1}{2}} \nabla u_{1}\right\|^{2} \\
& \leq\left(\frac{1}{\pi} \log \alpha^{-1}+\mathcal{O}(1)\right) \frac{1}{3}\left\|\chi\left(h_{1}<c\right)\left(h_{1}+\frac{1}{4}\right)^{\frac{1}{2}} \nabla u_{1}\right\|^{2} \text {. }
\end{aligned}
$$

The inequalities (220) and (221) prove the first equality of the Lemma.

The proofs of the last two equalities are similar but simpler.

\section{Lemma C.6.}

$$
\begin{aligned}
\left\|\left(H_{f}+P_{f}^{2}\right)^{-1} A^{+} \cdot A^{+} \Phi_{\sharp}^{u_{\alpha}}\right\|_{*}^{2}-\left\|\Phi_{*}^{2}\right\|_{*}^{2}\left\|\Phi_{\sharp}^{u_{\alpha}}\right\|^{2} & =o\left(\alpha^{3} \log \alpha^{-1}\right), \\
\left\|\left(H_{f}+P_{f}^{2}\right)^{-1} A^{+} \cdot A^{+} \Phi_{\sharp}^{u_{\alpha}}\right\|^{2} & =\mathcal{O}\left(\alpha^{3} \log \alpha^{-1}\right) \\
\left\|\left|k_{1}\right|^{\frac{1}{6}}\left|k_{2}\right|^{\frac{1}{6}}\left|k_{3}\right|^{\frac{1}{6}}\left(H_{f}+P_{f}^{2}\right)^{-1} A^{+} \cdot A^{+} \Phi_{\sharp}^{u_{\alpha}}\right\|^{2} & =\mathcal{O}\left(\alpha^{3}\right) \\
\left\|\left(h_{\alpha}+e_{0}\right)\left(H_{f}+P_{f}^{2}\right)^{-1} A^{+} \cdot A^{+} \Phi_{\sharp}^{u_{\alpha}}\right\|^{2} & =\mathcal{O}\left(\alpha^{7} \log \alpha^{-1}\right) .
\end{aligned}
$$

Proof. Denoting by $\sigma_{n}$ the set of all permutations of $\{1,2, \ldots, n\}$, we have

$$
\begin{aligned}
\left\|\left(H_{f}+P_{f}^{2}\right)^{-1} A^{+} \cdot A^{+} \Phi_{\sharp}^{u_{\alpha}}\right\|_{*}^{2} \\
=\frac{4 \alpha}{(2 \pi)^{3}} \| \frac{1}{\sqrt{6}} \sum_{(i, j, n) \in \sigma_{n}} \frac{\sum_{\lambda=1,2} \varepsilon_{\lambda}\left(k_{i}\right) \cdot \sum_{\nu=1,2} \varepsilon_{\nu}\left(k_{j}\right)}{\left(\sum_{p=1}^{3}\left|k_{p}\right|+\left(\sum_{p=1}^{3} k_{p}\right)^{2}\right)^{\frac{1}{2}}} \\
\quad \times \frac{\sum_{\kappa=1,2} \varepsilon_{\kappa}\left(k_{n}\right) \chi_{\Lambda}\left(\left|k_{1}\right|\right) \chi_{\Lambda}\left(\left|k_{2}\right|\right) \chi_{\Lambda}\left(\left|k_{3}\right|\right)}{\left|k_{i}\right|^{\frac{1}{2}}\left|k_{j}\right|^{\frac{1}{2}}\left|k_{n}\right|^{\frac{1}{2}}\left(\left|k_{n}\right|+k_{n}^{2}+\left(h_{\alpha}+e_{0}\right)\right)} \nabla u_{\alpha} \|^{2}
\end{aligned}
$$

If we pick two triples $(i, j, n)$ and $\left(i^{\prime}, j^{\prime}, n^{\prime}\right)$, such that $n \neq n^{\prime}$, then we get a product which is integrable at $k_{1}=k_{2}=k_{3}=0$, even without the term $\left(h_{\alpha}+e_{0}\right)$. The contribution of such terms is $c \alpha\left\|\nabla u_{\alpha}\right\|^{2}=\mathcal{O}\left(\alpha^{3}\right)$. Moreover, 
using the symmetry in $k_{1}, k_{2}, k_{3}$, the 12 remaining terms give the same contribution. This yields

$$
\begin{aligned}
& \left\|\left(H_{f}+P_{f}^{2}\right)^{-1} A^{+} \cdot A^{+} \Phi_{\sharp}^{u_{\alpha}}\right\|_{*}^{2}=\frac{8 \alpha}{(2 \pi)^{3}} \\
& \quad \times\left\|\frac{\sum_{\lambda} \varepsilon_{\lambda}\left(k_{3}\right) \cdot \sum_{\nu} \varepsilon_{\nu}\left(k_{2}\right) \sum_{\kappa} \varepsilon_{\kappa}\left(k_{1}\right) \chi_{\Lambda}\left(\left|k_{1}\right|\right) \chi_{\Lambda}\left(\left|k_{2}\right|\right) \chi_{\Lambda}\left(\left|k_{3}\right|\right)}{\left(\sum_{i=1}^{3}\left|k_{i}\right|+\left(\sum_{i=1}^{3} k_{i}\right)^{2}\right)^{\frac{1}{2}}\left|k_{3}\right|^{\frac{1}{2}}\left|k_{2}\right|^{\frac{1}{2}}\left|k_{1}\right|^{\frac{1}{2}}\left(\left|k_{1}\right|+k_{1}^{2}+\left(h_{\alpha}+e_{0}\right)\right)} \cdot \nabla u_{\alpha}\right\|^{2} \\
& \quad+\mathcal{O}\left(\alpha^{3}\right) .
\end{aligned}
$$

On the other hand, we have

$$
\begin{aligned}
& \left\|\Phi_{*}^{2}\right\|_{*}^{2}\left\|\Phi_{\#}^{u_{\alpha}}\right\|^{2}=\frac{8 \alpha}{(2 \pi)^{3}} \\
& \quad \times\left\|\frac{\sum_{\lambda} \varepsilon_{\lambda}\left(k_{3}\right) \cdot \sum_{\nu} \varepsilon_{\nu}\left(k_{2}\right) \sum_{\kappa} \varepsilon_{\kappa}\left(k_{1}\right) \chi_{\Lambda}\left(\left|k_{1}\right|\right) \chi_{\Lambda}\left(\left|k_{2}\right|\right) \chi_{\Lambda}\left(\left|k_{3}\right|\right)}{\left(\left|k_{2}\right|+\left|k_{3}\right|+\left(k_{2}+k_{3}\right)^{2}\right)^{\frac{1}{2}}\left|k_{3}\right|^{\frac{1}{2}}\left|k_{2}\right|^{\frac{1}{2}}\left|k_{1}\right|^{\frac{1}{2}}\left(\left|k_{1}\right|+k_{1}^{2}+\left(h_{\alpha}+e_{0}\right)\right)} \cdot \nabla u_{\alpha}\right\|^{2} .
\end{aligned}
$$

Therefore, we obtain

$$
\begin{aligned}
& \left\|\left(H_{f}+P_{f}^{2}\right)^{-1} A^{+} \cdot A^{+} \Phi_{\sharp}^{u_{\alpha}}\right\|_{*}^{2}-\left\|\Phi_{*}^{2}\right\|_{*}^{2}\left\|\Phi_{\sharp}^{u_{\alpha}}\right\|^{2}=-\frac{8 \alpha}{(2 \pi)^{3}} \\
& \quad \times \int\left(\frac{\left(\left|k_{1}\right|^{2}+2\left|k_{1}\right|\left|k_{3}\right|+2\left|k_{1}\right|\left|k_{2}\right|\right)}{\left|k_{2}\right|\left|k_{3}\right|\left(\left|k_{1}\right|+\left|k_{2}\right|+\left|k_{3}\right|+\left|k_{1}+k_{2}+k_{3}\right|^{2}\right)\left(\left|k_{2}\right|+\left|k_{3}\right|+\left|k_{2}+k_{3}\right|^{2}\right)}\right. \\
& \left.\quad \times \sum_{\lambda} \varepsilon_{\lambda}\left(k_{3}\right) \cdot \sum_{\nu} \varepsilon_{\nu}\left(k_{2}\right) \chi_{\Lambda}\left(\left|k_{1}\right|\right) \chi_{\Lambda}\left(\left|k_{2}\right|\right)^{2} \chi_{\Lambda}\left(\left|k_{3}\right|\right)^{2}\left|u\left(k_{1}, x\right)\right|^{2} \mathrm{~d} k_{1} \mathrm{~d} k_{2} \mathrm{~d} k_{3} \mathrm{~d} x\right),
\end{aligned}
$$

where

$$
u\left(k_{1}, x\right)=\frac{\sum_{\kappa} \varepsilon_{\kappa}\left(k_{1}\right) \chi_{\Lambda}\left(\left|k_{1}\right|\right)^{\frac{1}{2}}}{\left|k_{1}\right|^{\frac{1}{2}}\left(\left|k_{1}\right|+k_{1}^{2}+\left(h_{\alpha}+e_{0}\right)\right)} \cdot \nabla u_{\alpha} .
$$

For fixed $\delta$, we first compute the integral in (227) over the regions $\left|k_{1}\right|>\delta$. This yields a term $c_{\delta} \alpha^{3}$, where $c_{\delta}$ is independent on $\alpha$.

Next, integrating (227) over the regions $\left|k_{1}\right| \leq \delta$ yields a bound $\mathcal{O}(\delta) \alpha^{3} \log \alpha$, with $\mathcal{O}(\delta)$ independent of $\alpha$.

This concludes the proof of (222).

The proof of (223) is a straightforward computation showing

$$
\begin{aligned}
& \left\|\left(H_{f}+P_{f}^{2}\right)^{-1} A^{+} \cdot A^{+} \Phi_{\sharp}^{u_{\alpha}}\right\|^{2}=\frac{8 \alpha}{(2 \pi)^{3}} \\
& \quad \times\left\|\frac{\sum_{\lambda} \varepsilon_{\lambda}\left(k_{3}\right) \cdot \sum_{\nu} \varepsilon_{\nu}\left(k_{2}\right) \sum_{\kappa} \varepsilon_{\kappa}\left(k_{1}\right) \chi_{\Lambda}\left(\left|k_{1}\right|\right) \chi_{\Lambda}\left(\left|k_{2}\right|\right) \chi_{\Lambda}\left(\left|k_{3}\right|\right)}{\left(\sum_{i=1}^{3}\left|k_{i}\right|+\left(\sum_{i=1}^{3} k_{i}\right)^{2}\right)\left|k_{3}\right|^{\frac{1}{2}}\left|k_{2}\right|^{\frac{1}{2}}\left|k_{1}\right|^{\frac{1}{2}}\left(\left|k_{1}\right|+k_{1}^{2}+\left(h_{\alpha}+e_{0}\right)\right)} \cdot \nabla u_{\alpha}\right\|^{2} \\
& =\mathcal{O}\left(\alpha^{3} \log \alpha^{-1}\right) .
\end{aligned}
$$

The proofs of (224) and (225) are similar to those above. 
Lemma C.7. For $\Psi_{1}^{\perp}$ and $\Psi_{2}^{\perp}$ given in Definition 6.1, we have

$$
\left|2 \alpha \operatorname{Re}\left\langle A^{-} \cdot A^{-} \pi^{3} \Psi_{1}^{\perp}, \pi^{1} \Psi_{2}^{\perp}\right\rangle\right| \leq c \alpha^{5}+\epsilon\left\|H_{f}^{\frac{1}{2}} \Psi_{2}^{\perp}\right\|^{2} .
$$

Proof. Using $2 \alpha \operatorname{Re}\left\langle A^{-} \cdot A^{-} \pi^{3} \Psi_{1}^{\perp}, \pi^{1} \Psi_{2}^{\perp}\right\rangle=2 \alpha \operatorname{Re}\left\langle\pi^{3} \Psi_{1}^{\perp}, A^{+} . A^{+} \pi^{1} \Psi_{2}^{\perp}\right\rangle$, we obtain that $2 \alpha \operatorname{Re}\left\langle A^{-} . A^{-} \pi^{3} \Psi_{1}^{\perp}, \pi^{1} \Psi_{2}^{\perp}\right\rangle$ can be rewritten as a linear combination of the following two integrals $I_{1}$ and $I_{2}$ :

$$
\begin{aligned}
I_{1}= & \alpha^{\frac{5}{2}} \int \frac{\mathrm{d} k \mathrm{~d} k^{\prime} \mathrm{d} k^{\prime \prime} \mathrm{d} x}{|k|+\left|k^{\prime}\right|+\left|k^{\prime \prime}\right|+\left|k+k^{\prime}+k^{\prime \prime}\right|^{2}} \sum_{\kappa, \nu} \frac{\epsilon_{\kappa}\left(k^{\prime}\right) \cdot \epsilon_{\nu}\left(k^{\prime \prime}\right)}{\left|k^{\prime}\right|^{\frac{1}{2}}\left|k^{\prime \prime}\right|^{\frac{1}{2}}} \\
& \times\left(\frac{1}{|k|+|k|^{2}+\left(h_{\alpha}+e_{0}\right)} \sum_{\lambda} \frac{\epsilon_{\lambda}(k)}{|k|^{\frac{1}{2}}} \nabla u_{\alpha}\right) \sum_{\kappa, \eta} \frac{\epsilon_{\kappa}\left(k^{\prime}\right) \cdot \epsilon_{\eta}\left(k^{\prime \prime}\right)}{\left|k^{\prime}\right|^{\frac{1}{2}}\left|k^{\prime \prime}\right|^{\frac{1}{2}}} \overline{\left(\pi^{1} \Psi_{2}^{\perp}\right)(k, x),}
\end{aligned}
$$

and $I_{2}$ is defined as $I_{1}$, except that in the last sum, we reverse the role of $k$ and $k^{\prime \prime}$, namely

$$
\begin{aligned}
I_{2}= & \alpha^{\frac{5}{2}} \int \frac{\mathrm{d} k \mathrm{~d} k^{\prime} \mathrm{d} k^{\prime \prime} \mathrm{d} x \chi_{\Lambda}(|k|) \chi_{\Lambda}\left(\left|k^{\prime}\right|\right) \chi_{\Lambda}\left(\left|k^{\prime \prime}\right|\right)}{|k|+\left|k^{\prime}\right|+\left|k^{\prime \prime}\right|+\left|k+k^{\prime}+k^{\prime \prime}\right|^{2}} \sum_{\kappa, \nu} \frac{\epsilon_{\kappa}\left(k^{\prime}\right) \cdot \epsilon_{\nu}\left(k^{\prime \prime}\right)}{\left|k^{\prime}\right|^{\frac{1}{2}}\left|k^{\prime \prime}\right|^{\frac{1}{2}}} \\
& \times\left(\frac{1}{|k|+|k|^{2}+\left(h_{\alpha}+e_{0}\right)} \sum_{\lambda} \frac{\epsilon_{\lambda}(k)}{|k|^{\frac{1}{2}}} \nabla u_{\alpha}\right) \sum_{\kappa, \eta} \frac{\epsilon_{\kappa}\left(k^{\prime}\right) \cdot \epsilon_{\eta}(k)}{\left|k^{\prime}\right|^{\frac{1}{2}}|k|^{\frac{1}{2}}} \overline{\left(\pi^{1} \Psi_{2}^{\perp}\right)\left(k^{\prime \prime}, x\right)} .
\end{aligned}
$$

To bound $I_{2}$, we use the Schwarz inequality, $|a b| \leq \frac{1}{2 \epsilon} a^{2}+\frac{\epsilon}{2} b^{2}$. This yields

$$
\begin{aligned}
\left|I_{2}\right| \leq & \alpha^{\frac{5}{2}}\left(\int \frac{\mathrm{d} k \mathrm{~d} k^{\prime} \mathrm{d} k^{\prime \prime} \mathrm{d} x \chi_{\Lambda}^{2}(|k|) \chi_{\Lambda}^{2}\left(\left|k^{\prime}\right|\right) \chi_{\Lambda}^{2}\left(\left|k^{\prime \prime}\right|\right)}{\left(|k|+\left|k^{\prime}\right|+\left|k^{\prime \prime}\right|+\left|k+k^{\prime}+k^{\prime \prime}\right|^{2}\right)^{2}} \frac{2}{\left|k^{\prime}\right|\left|k^{\prime \prime}\right|}\right. \\
& \left.\times\left|\frac{1}{|k|+|k|^{2}+\left(h_{\alpha}+e_{0}\right)} \frac{1}{|k|^{\frac{1}{2}}} \nabla u_{\alpha}\right|^{2}|k|^{2-\epsilon}\left|k^{\prime}\right|^{2-\epsilon} \frac{1}{\left|k^{\prime \prime}\right|}\right)^{\frac{1}{2}} \\
& \times\left(2 \int \frac{\mathrm{d} k \mathrm{~d} k^{\prime} \mathrm{d} k^{\prime \prime} \mathrm{d} x}{\left|k^{\prime}\right||k|}\left|\left(\pi^{1} \Psi_{2}^{\perp}\right)\left(k^{\prime \prime}, x\right)\right|^{2}\left|k^{\prime \prime}\right| \frac{\chi_{\Lambda}^{2}(|k|) \chi_{\Lambda}^{2}\left(\left|k^{\prime}\right|\right) \chi_{\Lambda}^{2}\left(\left|k^{\prime \prime}\right|\right)}{|k|^{2-\epsilon}\left|k^{\prime}\right|^{2-\epsilon}}\right)^{\frac{1}{2}} \\
\leq & c \alpha^{7}+\epsilon\left\|H_{f}^{\frac{1}{2}} \Psi_{2}^{\perp}\right\|^{2},
\end{aligned}
$$

Similarly, we bound $I_{1}$ as follows:

$$
\begin{aligned}
\left|I_{1}\right| \leq & \alpha^{\frac{5}{2}}\left(\int \frac{\mathrm{d} k \mathrm{~d} k^{\prime} \mathrm{d} k^{\prime \prime} \mathrm{d} x}{\left(|k|+\left|k^{\prime}\right|+\left|k^{\prime \prime}\right|+\left|k+k^{\prime}+k^{\prime \prime}\right|^{2}\right)^{2}} \frac{2}{\left|k^{\prime}\right|\left|k^{\prime \prime}\right|}\right. \\
& \left.\times\left|\frac{1}{|k|+|k|^{2}+\left(h_{\alpha}+e_{0}\right)} \frac{1}{|k|^{\frac{1}{2}}} \nabla u_{\alpha}\right|^{2}\left|k^{\prime}\right|^{2-\epsilon}\left|k^{\prime \prime}\right|^{2-\epsilon} \frac{\chi_{\Lambda}^{2}(|k|) \chi_{\Lambda}^{2}\left(\left|k^{\prime}\right|\right) \chi_{\Lambda}^{2}\left(\left|k^{\prime \prime}\right|\right)}{|k|}\right)^{\frac{1}{2}} \\
& \times\left(2 \int \frac{\mathrm{d} k \mathrm{~d} k^{\prime} \mathrm{d} k^{\prime \prime} \mathrm{d} x}{\left|k^{\prime}\right|\left|k^{\prime \prime}\right|}\left|\left(\pi^{1} \Psi_{2}^{\perp}\right)(k, x)\right|^{2}|k| \frac{\chi_{\Lambda}^{2}(|k|) \chi_{\Lambda}^{2}\left(\left|k^{\prime}\right|\right) \chi_{\Lambda}^{2}\left(\left|k^{\prime \prime}\right|\right)}{\left|k^{\prime}\right|^{2-\epsilon}\left|k^{\prime \prime}\right|^{2-\epsilon}}\right)^{\frac{1}{2}} \\
\leq & c \alpha^{5} \int \mathrm{d} k^{\prime} \mathrm{d} k^{\prime \prime}\left|k^{\prime}\right|^{-\epsilon}\left|k^{\prime \prime}\right|^{-\epsilon} \chi_{\Lambda}^{2}\left(\left|k^{\prime}\right|\right) \chi_{\Lambda}^{2}\left(\left|k^{\prime \prime}\right|\right) \int \mathrm{d} k \frac{1}{|k|^{2}} \frac{\chi_{\Lambda}^{2}(|k|)}{\left(|k|+\frac{1}{16} \alpha^{2}\right)^{2}}\left\|\nabla u_{\alpha}\right\|^{2} \\
& +\epsilon\left\|H_{f}^{\frac{1}{2}} \pi^{1} \Psi_{2}^{\perp}\right\|^{2} \leq c \alpha^{5}+\epsilon\left\|H_{f}^{\frac{1}{2}} \Psi_{2}^{\perp}\right\|^{2},
\end{aligned}
$$


where we took into account that $\frac{\partial u_{\alpha}}{\partial x_{i}}$ is orthogonal to $u_{\alpha}$, and on the subspace of such functions we have $\left(h-\alpha+e_{0}\right) \geq \frac{1}{16} \alpha^{2}$.

\section{References}

[1] Bach, V., Chen, T., Fröhlich, J., Sigal, I.M.: The renormalized electron mass in non-relativistic QED. J. Funct. Anal. 243(2), 426-535 (2007)

[2] Bach, V., Fröhlich, J., Pizzo, A.: Infrared-finite algorithms in QED: the groundstate of an atom interacting with the quantized radiation field. Commun. Math. Phys. 264(1), 145-165 (2006)

[3] Bach, V., Fröhlich, J., Sigal, I.M.: Spectral analysis for systems of atoms and molecules coupled to the quantized radiation field. Commun. Math. Phys. 207(2), 249-290 (1999)

[4] Bach, V., Fröhlich, J., Sigal, I.M.: Quantum electrodynamics of confined relativistic particles. Adv. Math. 137(2), 299-395 (1998)

[5] Bach, V., Fröhlich, J., Sigal, I.M.: Mathematical theory of radiation. Found. Phys. 27(2), 227-237 (1997)

[6] Barbaroux, J.-M., Vugalter, S.: Non-analyticity of the ground state energy of the Hydrogen atom in nonrelativistic QED. J. Phys. A: Math. Theor. 43, 474004 (2010)

[7] Barbaroux, J.-M., Chen, T., Vougalter, V., Vugalter, S.A.: On the ground state energy of the translation invariant Pauli-Fierz model. Proc. Am. Math. Soc. 136, 1057-1064 (2008)

[8] Chen, T.: Infrared renormalization in non-relativistic QED and scaling criticality. J. Funct. Anal. 354(10), 2555-2647 (2008)

[9] Chen, T., Fröhlich, J.: Coherent infrared representations in non-relativistic QED. In: Spectral Theory and Mathematical Physics: A Festschrift in Honor of Barry Simon's 60th Birthday. Proc. Symp. Pure Math., vol. I. AMS (2007)

[10] Chen, T., Vougalter, V., Vugalter, S.A.: The increase of binding energy and enhanced binding in non-relativistic QED. J. Math. Phys. 44(5), 1961-1970 (2003)

[11] Fröhlich, J.: On the infrared problem in a model of scalar electrons and massless, scalar bosons. Ann. Inst. H. Poincaré Sect. A (N.S.) 19, 1-103 (1973)

[12] Griesemer, M., Hasler, D.: Analytic perturbation theory and renormalization analysis of matter coupled to quantized radiation. Ann. Henri Poincaré 10(3), 577-621 (2009)

[13] Griesemer, M., Lieb, E.H., Loss, M.: Ground states in non-relativistic quantum electrodnamics. Inv. Math 145, 557-595 (2001)

[14] Hainzl, C.: Enhanced binding through coupling to a photon field. Mathematical results in quantum mechanics (Taxco, 2001). Contemp. Math., vol. 307, pp. 149-154. Amer. Math. Soc., Providence (2002)

[15] Hainzl, C., Hirokawa, M., Spohn, H.: Binding energy for hydrogen-like atoms in the Nelson model without cutoffs. J. Funct. Anal. 220(2), 424-459 (2005)

[16] Hainzl, C., Vougalter, V., Vugalter, S.A.: Enhanced binding in non-relativistic QED. Commun. Math. Phys. 233, 13-26 (2003) 
Jean-Marie Barbaroux

Centre de Physique Théorique

Luminy Case 907

13288 Marseille Cedex 9

France

and

Département de Mathématiques

Université du Sud Toulon-Var

83957 La Garde Cedex

France

e-mail: barbarou@univ-tln.fr

Thomas Chen

Department of Mathematics

University of Texas at Austin

1 University Station C1200

Austin

TX 78712

USA

e-mail: tc@math.utexas.edu

Vitali Vougalter

Department of Mathematics and Applied Mathematics

University of Cape Town

Private Bag

Rondebosch 7701

South Africa

e-mail: Vitali.Vougalter@uct.ac.za

Semjon Vugalter

Mathematisches Institut

Ludwig-Maximilians-Universität München

Theresienstrasse 39

80333 Munich

Germany

e-mail: wugalter@mathematik . uni-muenchen.de

Communicated by Volker Bach.

Received: March 26, 2009.

Accepted: October 1, 2010. 